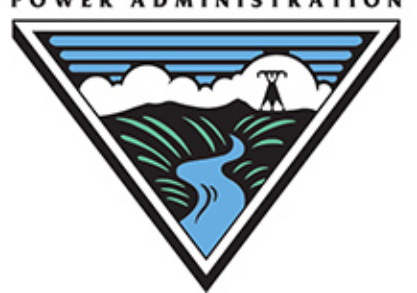

Pilot Testing of Commercial Refrigeration-Based Demand Response

\author{
A. Hirsch, J. Clark, M. Deru, K. Trenbath, \\ I. Doebber, and D. Studer
}

Produced under direction of the Bonneville Power Administration by the National Renewable Energy Laboratory (NREL) under Interagency Agreement IAG-14-1921 and Task No WFGA.1000.

NREL is a national laboratory of the U.S. Department of Energy Office of Energy Efficiency \& Renewable Energy Operated by the Alliance for Sustainable Energy, LLC

This report is available at no cost from the National Renewable Energy Laboratory (NREL) at www.nrel.gov/publications.

Strategic Partnership Project Report

NREL/TP-5500-65009

October 2015

Contract No. DE-AC36-08GO28308 


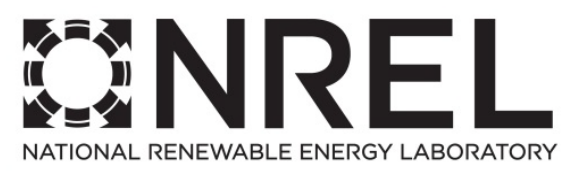

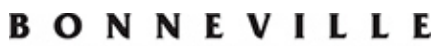

POWER ADMINISTRATION

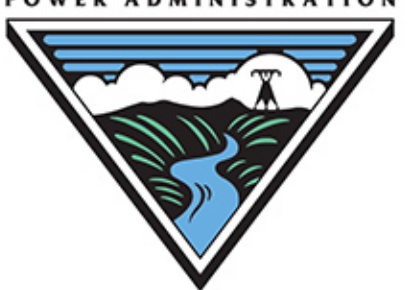

\title{
Pilot Testing of Commercial Refrigeration-Based Demand Response
}

\author{
A. Hirsch, J. Clark, M. Deru, K. Trenbath, \\ I. Doebber, and D. Studer
}

Prepared under Task No. WFGA.1000

NREL is a national laboratory of the U.S. Department of Energy Office of Energy Efficiency \& Renewable Energy Operated by the Alliance for Sustainable Energy, LLC

This report is available at no cost from the National Renewable Energy Laboratory (NREL) at www.nrel.gov/publications.

National Renewable Energy Laboratory 15013 Denver West Parkway Golden, CO 80401

303-275-3000 • www.nrel.gov
Strategic Partnership Project Report

NREL/TP-5500-65009

October 2015

Contract No. DE-AC36-08GO28308 


\section{NOTICE}

This manuscript has been authored by employees of the Alliance for Sustainable Energy, LLC ("Alliance") under Contract No. DE-AC36-08GO28308 with the U.S. Department of Energy (“DOE”).

This report was prepared as an account of work sponsored by an agency of the United States government. Neither the United States government nor any agency thereof, nor any of their employees, makes any warranty, express or implied, or assumes any legal liability or responsibility for the accuracy, completeness, or usefulness of any information, apparatus, product, or process disclosed, or represents that its use would not infringe privately owned rights. Reference herein to any specific commercial product, process, or service by trade name, trademark, manufacturer, or otherwise does not necessarily constitute or imply its endorsement, recommendation, or favoring by the United States government or any agency thereof. The views and opinions of authors expressed herein do not necessarily state or reflect those of the United States government or any agency thereof. 


\section{Acknowledgments}

The authors would like to thank the Bonneville Power Administration (BPA) for funding Technology Innovation Project \#307 under Interagency Agreement IAG-14-1921. In particular, the team would like to thank BPA Project Manager Jamie Anthony, Contracting Officer Matt DeLong, Contracting Officer's Technical Representative James Hillegas-Enting, Gary Smith, members of the BPA Demand Response Team_-Lee Hall, Frank Brown, and Thor Hinckleyand, finally, the BPA Technical Innovation Council. The authors would also like to acknowledge the contribution of the other project team members: Aaron Daly and Larry Rush (Whole Foods Market); Peter Radford, Michelle Collier, and Tony Ryan (Parasense); James Mitchell (Emerson Climate Technologies); and especially Ben Boyer (Source Refrigeration). 


\section{List of Acronyms}

BPA

Btu

CBL

COP

DAT

DR

EEPR

HVAC

$\mathrm{kW}$

MW

NREL

PRBS

PS

RMSE

SST

SCT

TAN

WFM
Bonneville Power Administration

British thermal unit

customer baseline load

coefficient of performance

discharge air temperature

demand response

electronic evaporator pressure regulator

heating, ventilating, and air conditioning

kilowatt

megawatt

National Renewable Energy Laboratory

pseudo-random binary signal

product simulator

root mean squared error

saturated suction temperature

saturated condensing temperature

Tanasbourne Whole Foods Market

Whole Foods Market 


\section{Executive Summary}

Supermarkets potentially offer a substantial demand response (DR) resource because of their high energy intensity and use patterns. This report describes a pilot project conducted to better estimate supermarket DR potential. Previous work has analyzed supermarket DR using heating, ventilating, and air conditioning (HVAC), lighting, and anti-condensate heaters. This project was concerned with evaluating DR using the refrigeration system and quantifying the DR potential inherent in supermarket refrigeration systems. Ancillary aims of the project were to identify practical barriers to the implementation of DR programs in supermarkets and to determine which high-level control strategies were most appropriate for achieving certain DR objectives. The scope of this project does not include an economic analysis of supermarket DR potential, detailed control strategy development for DR, or development of a strategy for regional implementation of DR in supermarkets.

\section{ES.1 Objectives}

The team pursued five objectives in this study:

1. Conduct pilot evaluation tests to understand stakeholder concerns and barriers to implementing DR in supermarket refrigeration systems.

2. In the course of these pilot tests, evaluate strategies that can provide 3- to 4-hour capacity reserves (identified as a key DR resource by the Bonneville Power Administration [BPA] for capacity management during critical demand peaks).

3. Conduct additional experiments in a real supermarket for the purpose of developing reliable models of the type needed to quantify DR potential.

4. Use these models to estimate the total aggregated supermarket refrigeration DR resource available to BPA.

5. Recommend future research and other work that must be done to maximize DR potential in supermarkets.

\section{ES.2 Methodology}

For this project, the team conducted all evaluation tests and experiments in an operational 45,591- $\mathrm{ft}^{2}$ supermarket located in Hillsboro, Oregon. The team conducted this work in three phases:

Phase I. Tests were conducted to establish the approximate timescale for DR using refrigerated display cases and walk-ins.

Phase II. Several evaluation tests of supermarket DR events were conducted. Throughout the testing phase the team worked closely with the store owner to understand their food safety, food integrity, and store operation concerns to ensure that the DR tests did not result in adverse outcomes. A summary of the tests is included in Tables ES-1. The team tested several types of control, including:

- Precooling the cases before the simulated DR event (indicated by the "Precool" column in Table ES-1). 
- Changing the discharge air temperature (DAT) set point for noncritical cases.

- Changing the DAT set point for the critical case.

- Controlling the compressor based on product simulator temperature rather than DAT.

Table ES-1. Site and Control Parameters for Each DR Test

\begin{tabular}{ccclllll}
\hline Test & Date & Store & Precool & $\begin{array}{c}\text { Change } \\
\text { Critical Case } \\
\text { DAT Set Point }\end{array}$ & $\begin{array}{c}\text { Change } \\
\text { Noncritical } \\
\text { Case DAT Set } \\
\text { Point }\end{array}$ & $\begin{array}{c}\text { DAT or } \\
\text { Product } \\
\text { Simulator } \\
\text { (PS) Control }\end{array}$ \\
\hline Test \#1 & $9 / 23 / 14$ & TAN $^{\mathrm{a}}$ & No & Yes & No & DAT \\
\hline Test \#2 & $11 / 10 / 14$ & TAN & No & Yes & No & PS \\
\hline Test \#3 & $7 / 14 / 15$ & TAN & No & Yes & No & DAT \\
\hline Test\#4 & $7 / 20 / 15$ & TAN & No & Yes & Yes & DAT \\
\hline Test \#5 & $7 / 22 / 15$ & TAN & Yes & Yes & Yes & DAT \\
\hline Test\#6 & $7 / 24 / 15$ & TAN & Yes & Yes & No & DAT \\
\hline Test \#7 & $7 / 28 / 15$ & TAN & No & No & Yes & DAT \\
\hline Test \#8 & $7 / 30 / 15$ & TAN & Yes & Yes & Yes & DAT \\
\hline Test \#9 & $7 / 31 / 15$ & TAN & Yes & No & No & DAT \\
\hline
\end{tabular}

${ }^{\text {a }}$ Tanasbourne Whole Foods Market

Phase III. Additional experiments were used to develop and calibrate models for analyzing situations not evaluated in the real store. The team then used these models to quantify the available DR resource in supermarket freezers in BPA's service territory. The team used the validated models to analyze three different situations: a DR event generated in response to a call 10 minutes before the event began, a DR event conducted in response to a call the day before the event, and a load-add event. The three types of simulations were conducted for four separate subregions of BPA's service territory.

\section{ES.3 Key Results}

This project had three key results:

- Initial tests established a rate of temperature increase in reach-in freezer cases and walkins when refrigeration was suspended. The reach-in cases warmed at a rate of approximately $10^{\circ} \mathrm{F} / \mathrm{h}$ when cooling to the case was suspended; the walk-ins warmed at a rate of approximately $2^{\circ} \mathrm{F} / \mathrm{h}$.

- The team conducted evaluation tests of DR events using different control strategies.

Despite being able to modify operation of less than $20 \%$ of the capacity of the refrigeration system, the team was able to demonstrate a load shed of around 10 kilowatts $(\mathrm{kW})$ for more than 2 hours in the most effective DR tests. Table ES-2 shows the results of the nine real-world DR evaluation tests. 
Table ES-2. Summary of Test Conditions and Resulting Load Shed for DR Evaluation Tests

\begin{tabular}{lccccccc}
\hline Test & $\begin{array}{c}\text { Load Shed } \\
\text { Range } \\
\mathbf{\pm 9 5 \%} \mathbf{( k W )}\end{array}$ & $\begin{array}{c}\text { Average } \\
\text { Load } \\
\mathbf{S h e d} \\
\mathbf{( k W )}\end{array}$ & $\begin{array}{c}\text { Outdoor Air } \\
\text { Temp. Range } \\
\left({ }^{\circ} \mathbf{F}\right)\end{array}$ & $\begin{array}{c}\text { Average } \\
\text { Outdoor } \\
\text { Air Temp. } \\
\mathbf{(} \mathbf{F})\end{array}$ & $\begin{array}{c}\text { Baseline } \\
\text { Load } \\
\text { Range } \\
\mathbf{( k W})\end{array}$ & $\begin{array}{c}\text { Average } \\
\text { Baseline } \\
\text { Load } \\
\mathbf{( k W )}\end{array}$ & $\begin{array}{c}\text { Average } \\
\text { Additional } \\
\text { Precool Power } \\
\text { Draw (kW) }\end{array}$ \\
\hline Test \#1 & $-0.4-8.0$ & 3.8 & $60.8-63.7$ & 62.3 & $22.5-29.0$ & 26.2 & N/A \\
\hline Test \#2 & $4.7-13.2$ & 9.0 & $59.7-61.7$ & 60.8 & $27.6-30.8$ & 28.9 & N/A \\
\hline Test \#3 & $-1.3-8.3$ & 3.5 & $87.4-92.3$ & 90.2 & $35.2-42.7$ & 38.9 & N/A \\
\hline Test \#4 & $3.2-13.6$ & 8.4 & $90.0-94.3$ & 92.2 & $35.5-44.3$ & 40.6 & N/A \\
\hline Test \#5 & $-1.9-9.2$ & 3.6 & $71.4-75.2$ & 73.8 & $29.1-35.9$ & 32.6 & 7.7 \\
\hline Test \#6 & $-3.3-7.6$ & 2.1 & $84.9-91.4$ & 88.0 & $35.4-42.0$ & 39.2 & 3.5 \\
\hline Test \#7 & $-3.1-6.8$ & 1.9 & $87.1-92.1$ & 89.3 & $35.5-41.8$ & 38.3 & N/A \\
\hline Test \#8 & $2.5-11.9$ & 7.2 & $95.5-111.9$ & 105.8 & $43.3-50.7$ & 47.2 & 4.6 \\
\hline Test \#9 & $-7.3-3.1$ & -2.1 & $104.4-108.5$ & 106.1 & $44.4-49.4$ & 46.7 & 5.7 \\
\hline
\end{tabular}

- The team simulated DR events using calibrated models to quantify available DR resources. The simulations showed that demand savings of 15.0 to $19.8 \mathrm{~kW}$ are available for 1.5 hours for a typical store without precooling and for about 2.5 hours with precooling throughout most of BPA's service territory, using only the low-temperature, non-ice-cream cases. The modeling study also showed that 5.2 to $5.5 \mathrm{~kW}$ of demand could be added for 3 hours per store, depending on location, under the same constraints. Table ES-3 shows the simulated available load shed or load add in each of the four representative cities simulated. These values assume only the low-temperature cases are available for DR, as was the case in the real store used for this project. 
Table ES-3. Summary of Results of Modeling Study for Four Representative Cities

\begin{tabular}{ll|cc|cc|cc}
\hline & \multicolumn{2}{c}{ Precool } & \multicolumn{2}{c}{ DR } & \multicolumn{2}{c}{ Rebound } \\
\hline Location & Simulation & $\begin{array}{c}\text { Additional } \\
\text { Load (kW) }\end{array}$ & $\begin{array}{c}\text { Time } \\
\text { (h) }\end{array}$ & $\begin{array}{c}\text { Load Shed } \\
\text { (kW) }\end{array}$ & $\begin{array}{c}\text { Time } \\
\text { (h) }\end{array}$ & $\begin{array}{c}\text { Additional } \\
\text { Load (kW) }\end{array}$ & $\begin{array}{c}\text { Time } \\
\text { (h) }\end{array}$ \\
Seattle & 10-Minute Notification & NA & NA & 18.5 & 1.25 & 6.5 & $3 \mathrm{~h}$ \\
\hline Spokane & 10-Minute Notification & NA & NA & 19.5 & 1.5 & 6.8 & 2.75 \\
\hline Portland & 10-Minute Notification & NA & NA & 15.0 & 1.5 & 5.3 & 2.75 \\
\hline Boise & 10-Minute Notification & NA & NA & 19.8 & 1.5 & 6.8 & 3 \\
\hline Seattle & Day-Before Notification & 6.8 & 4 & 17.8 & 2.5 & 6.6 & 3.5 \\
\hline Spokane & Day-Before Notification & 6.9 & 4 & 19.3 & 2.5 & 6.9 & 3.5 \\
\hline Portland & Day-Before Notification & 5.5 & 4 & 15.0 & 3 & 5.5 & 4 \\
\hline Boise & Day-Before Notification & 6.9 & 4 & 19.3 & 2.25 & 6.9 & 3.5 \\
\hline Seattle & Load-Add & 5.4 & 3 & N/A & N/A & -12.0 & 0.75 \\
\hline Spokane & Load-Add & 5.2 & 3 & N/A & N/A & -11.2 & 0.75 \\
\hline Portland & Load-Add & 5.5 & 3 & N/A & N/A & -12.0 & 0.75 \\
\hline Boise & Load-Add & 5.2 & 3 & N/A & N/A & -11.5 & 0.75 \\
\hline
\end{tabular}

\section{ES.4 Conclusions and Future Needs}

Through a set of field tests and a detailed modeling study, the team determined that a reasonable expectation for the amount of supermarket load shedding that may be offered by manipulating only low-temperature cases (excluding ice-cream cases) in a single typical store is on the order of 15 to $20 \mathrm{~kW}$ in the Pacific Northwest. Extrapolated to the entire region, this represents an aggregate potential on the order of 20 megawatts within BPA's service territory, again just from low-temperature, non-ice-cream cases. This DR resource should be available for 1.5 hours with no precooling, and for more than 2 hours when cases are cooled below their normal temperatures before the DR event.

Inability to shed refrigeration load for medium-temperature products is the largest barrier to realizing more significant DR for supermarkets, because medium-temperature cases account for a majority of the electricity consumption in a typical supermarket. Medium-temperature products must be kept within a tighter temperature range than low-temperature products, and for this reason store owners are reluctant to allow medium-temperature case set point changes.

Laboratory tests of medium-temperature case DR strategies are needed so that owners become comfortable testing, and ultimately implementing, medium-temperature case DR. The nextlargest barrier is lack of control over ancillary reach-in case equipment, such as anti-sweat heaters, lights, and fans. Control of such equipment is necessary to implement DR strategies that achieve greater load shed, but control of this equipment is not possible in most supermarket displays today.

Based on this study, the team recommends the following modifications be implemented in stores interested in participating in refrigeration DR: 
1. Assemble cases into suction groups to allow greater freedom to vary set points.

2. Enable floating suction control and possibly even steady-state case-level control to be switched between case air temperature and product simulators.

3. Implement variable capacity compressors to maintain low and predictable power consumption during DR events.

4. Better insulate reach-in cases to decrease external thermal loads.

5. Enable refrigeration lights, fans, and anti-condensate heaters to ramp down for DR in concert with the refrigeration system.

The team also identified several research needs for ensuring DR potential in supermarkets is maximized:

1. Conduct a laboratory study of DR using medium-temperature cases.

2. Conduct real-world and modeling studies to determine the coupled effect of variation of HVAC set points and refrigeration set points.

3. Conduct research in concert with food safety experts to better understand the constraints under which DR should be conducted in regard to food safety.

4. Further investigate the relationship between adding load at times when power is readily available and reducing power when it is not.

5. Research active phase-change solutions to augment dispatchable thermal storage capabilities. 


\section{Table of Contents}

Executive Summary

ES.1 Objectives

ES.2 Methodology

ES.3 Key Results

ES. 4 Conclusions and Future Needs

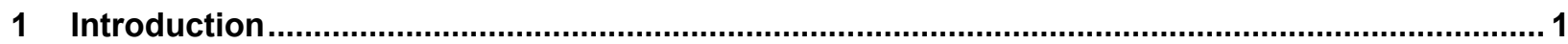

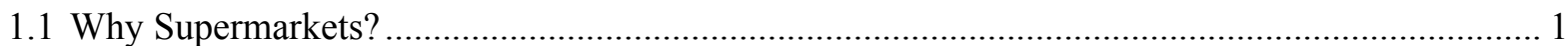

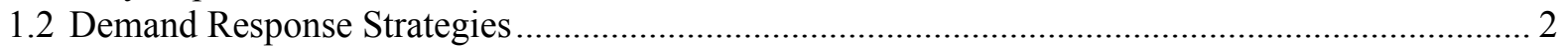

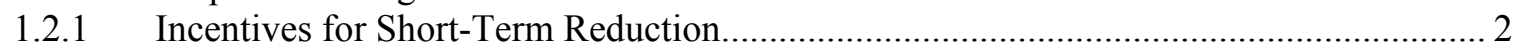

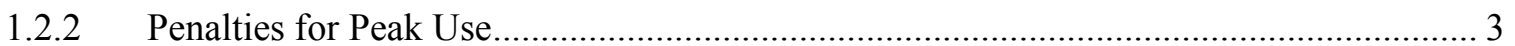

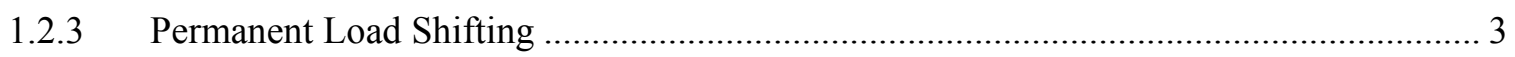

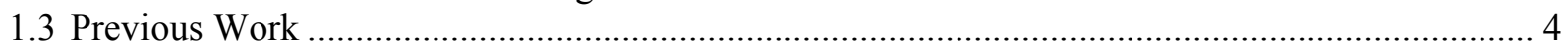

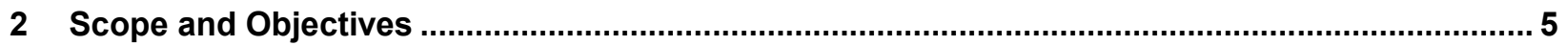

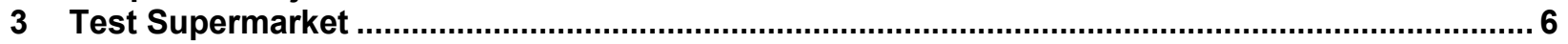

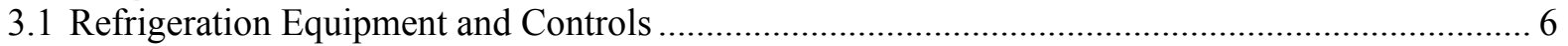

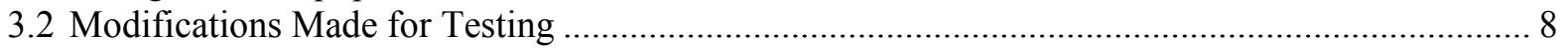

3.2.1 Electrical Submeter Installation and Commissioning .............................................. 8

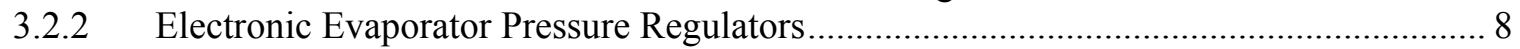

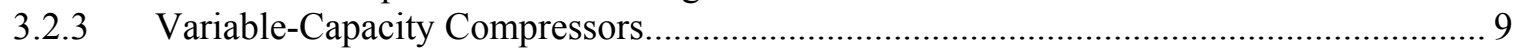

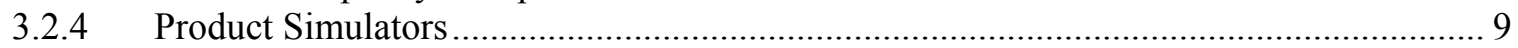

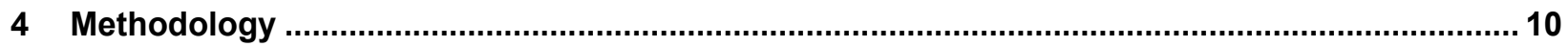

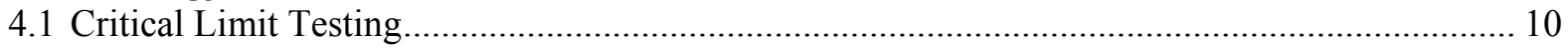

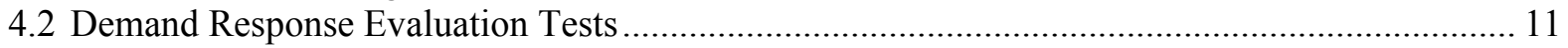

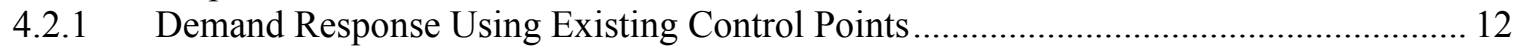

4.2.2 Demand Response Using Product Simulators.......................................................... 12

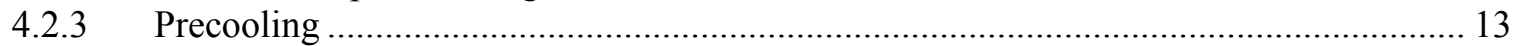

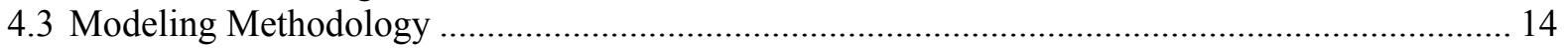

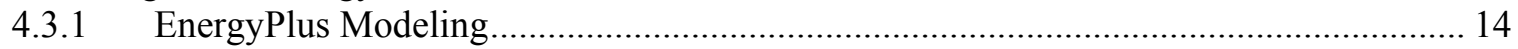

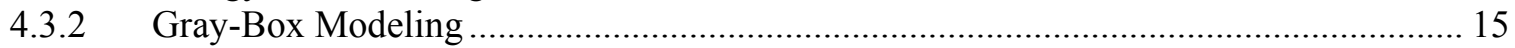

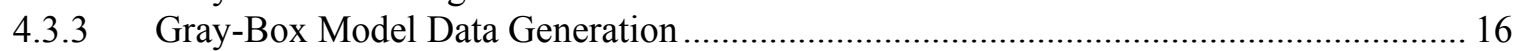

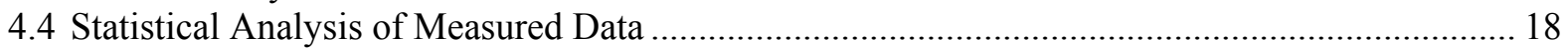

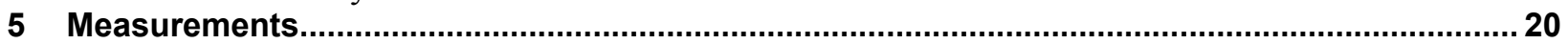

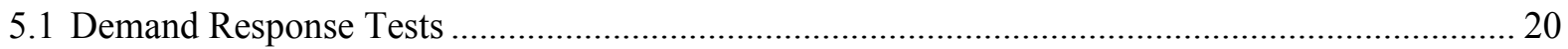

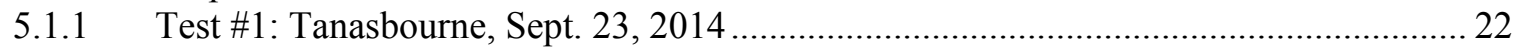

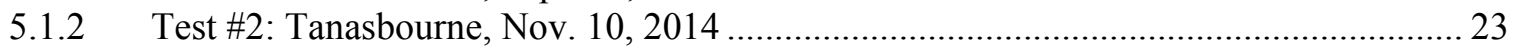

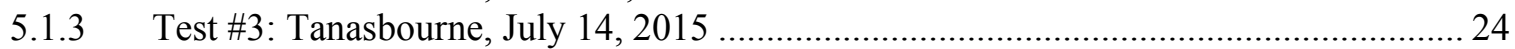

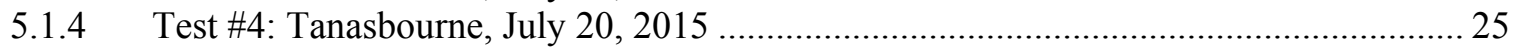

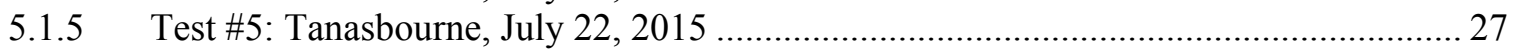

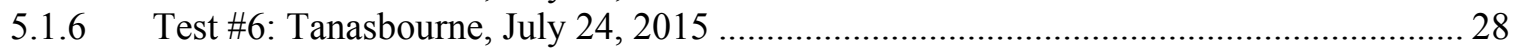

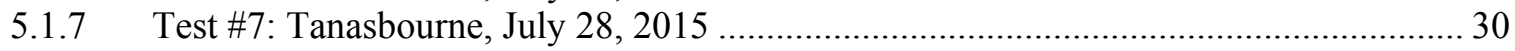

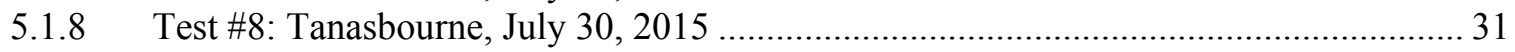

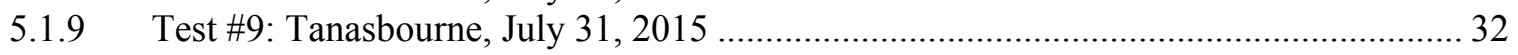

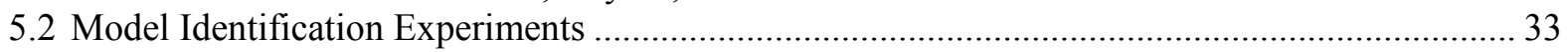

5.2.1 Tanasbourne Pseudo-Random Binary Signal Training, July 21, 2015 ......................... 34

5.2.2 Tanasbourne Pseudo-Random Binary Signal Validation, July 23, 2015 ...................... 36

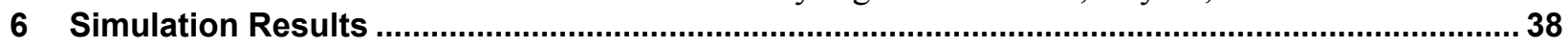

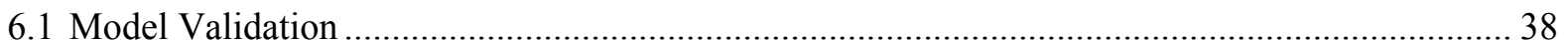

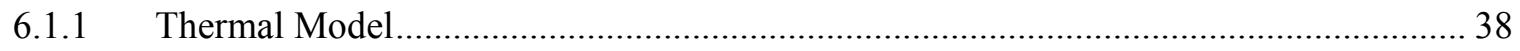

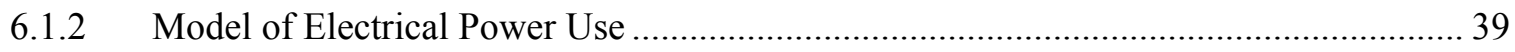




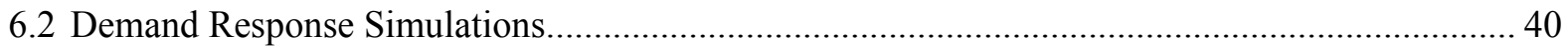

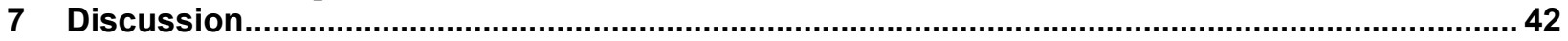

7.1 Thermal Dynamics of Refrigerated Display Cases and Walk-Ins ............................................ 42

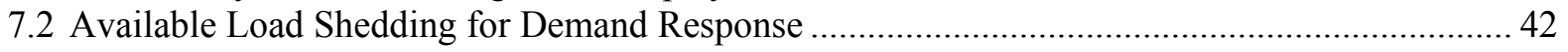

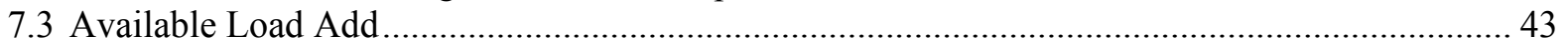

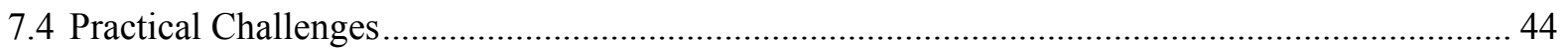

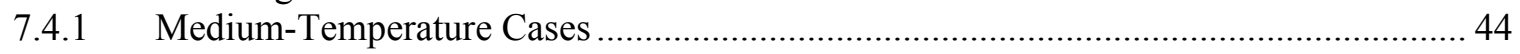

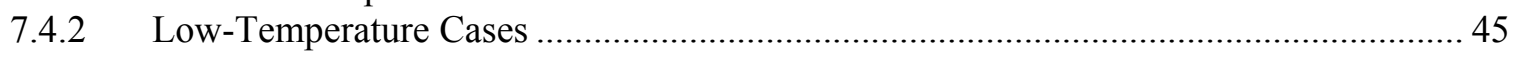

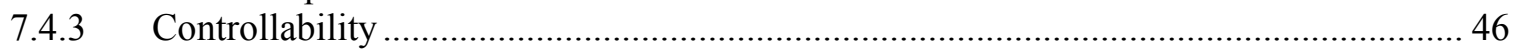

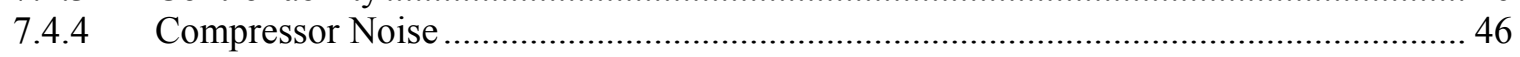

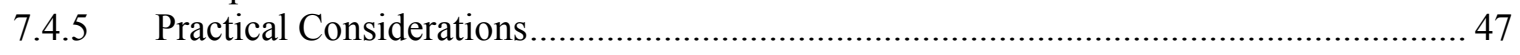

7.5 Considerations for Choosing a Demand Response Strategy ......................................................... 47

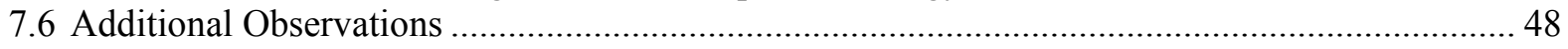

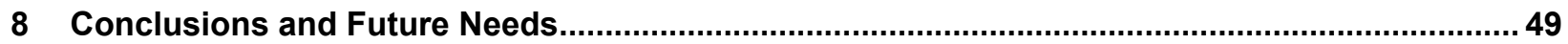

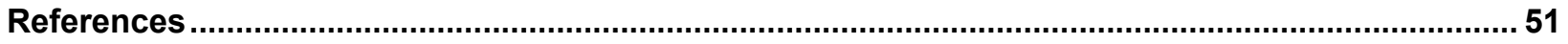

Appendix

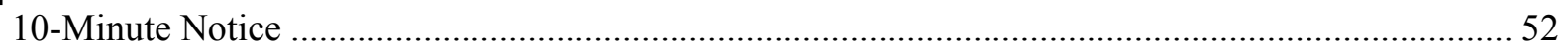

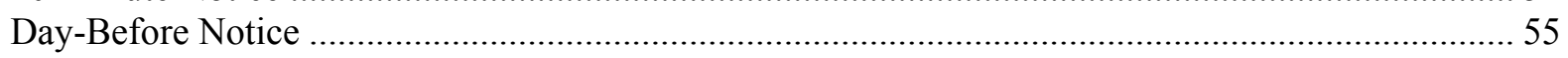

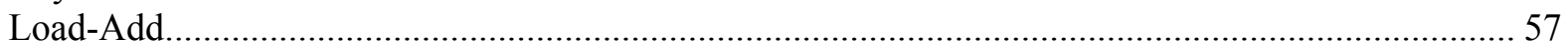




\section{List of Figures}

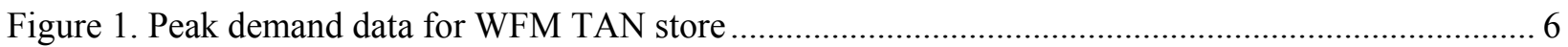

Figure 2. Photo of the product simulator, controller, and radio unit....................................................... 9

Figure 3. Reach-in frozen food freezer product simulator temperature rise with time ............................. 10

Figure 4. Meat walk-in freezer product simulator temperature rise with time ...................................... 11

Figure 5. Illustration showing typical refrigeration system configuration............................................ 12

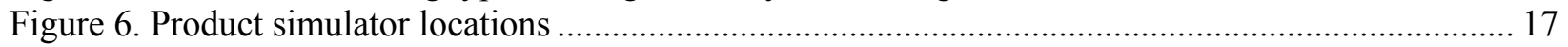

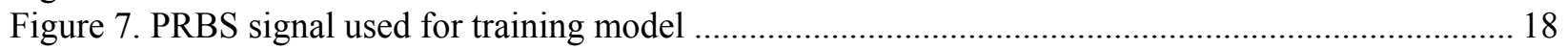

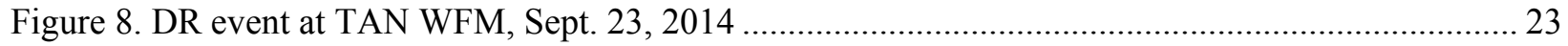

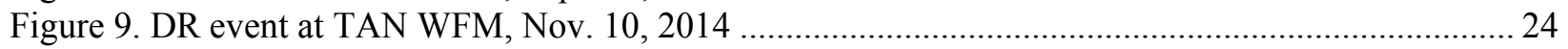

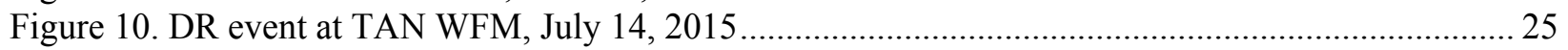

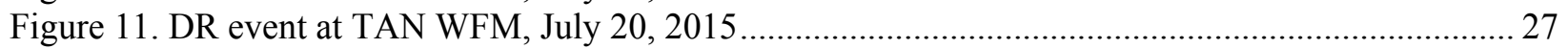

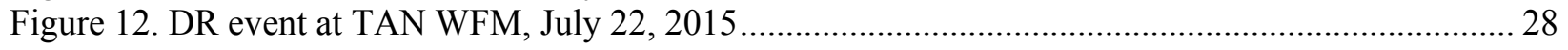

Figure 13. DR event at TAN WFM, July 24, 2015 ................................................................. 30

Figure 14. Demand response event at Tanasbourne Whole Foods Market, July 28, 2015 ....................... 31

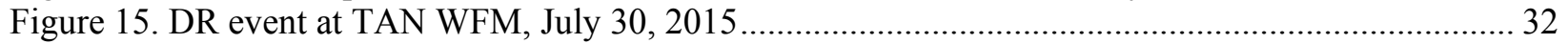

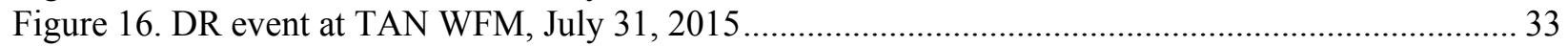

Figure 17. Walk-in air temperature and set point during training test .............................................. 34

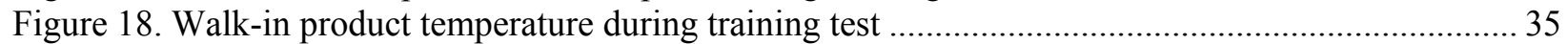

Figure 19. Reach-in air temperature and set point during training test ................................................. 35

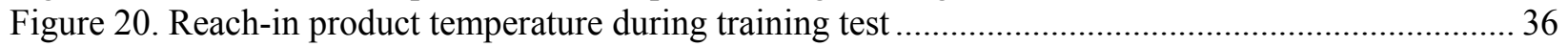

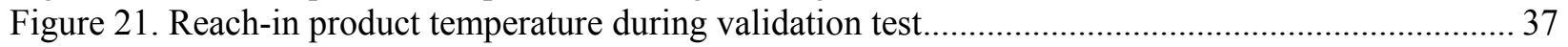

Figure 22. Walk-in product temperature during validation test ............................................................. 37

Figure 23. Reach-in validation: maximum temperature comparison $(\mathrm{RMSE}=0.401) \ldots \ldots \ldots \ldots \ldots \ldots \ldots \ldots . . . \ldots \ldots$

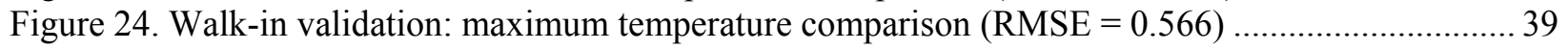

Figure 25. Comparison between modeled and "measured" electrical power during demand response...... 39

Figure 26. Reference supermarket refrigeration electricity load at $90^{\circ} \mathrm{F}$ condensing temperature, by case

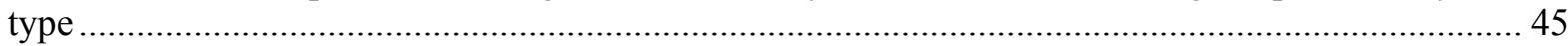

Figure 27. Simulated 10-minute notice Seattle, Washington, DR event................................................ 52

Figure 28. Simulated 10-minute notice Spokane, Washington, DR event.............................................. 53

Figure 29. Simulated 10-minute notice Portland, Oregon, DR event .................................................... 53

Figure 30. Simulated 10-minute notice Boise, Idaho, DR event.......................................................... 54

Figure 31. Simulated day-before notice Seattle, Washington, DR event................................................ 55

Figure 32. Simulated day-before notice Spokane, Washington, DR event............................................. 55

Figure 33. Simulated day-before notice Portland, Oregon, DR event .................................................. 56

Figure 34. Simulated day-before notice Boise, Idaho, DR event............................................................ 56

Figure 35. Simulated load-add event, Seattle, Washington ............................................................... 57

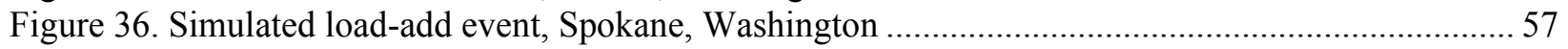

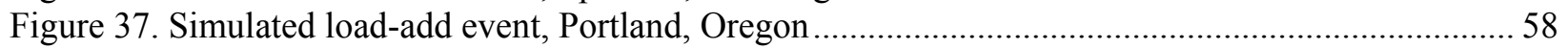

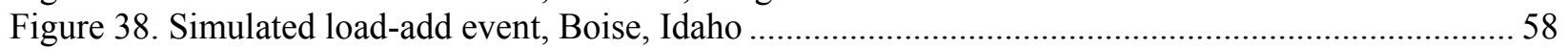




\section{List of Tables}

Table 1. TAN Low-Temperature Refrigeration System Circuits...................................................... 7

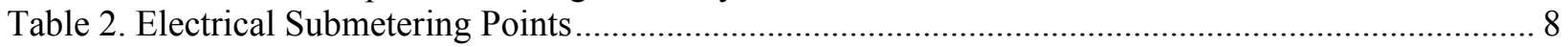

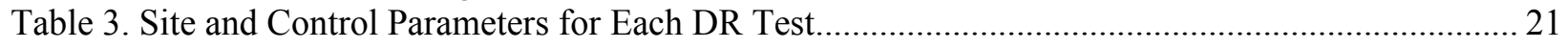

Table 4. DR Results for Each Evaluation Test ................................................................................ 21

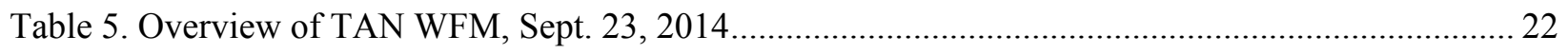

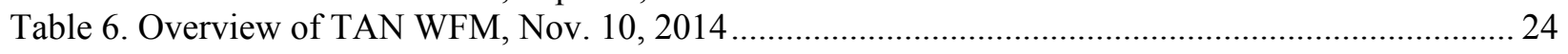

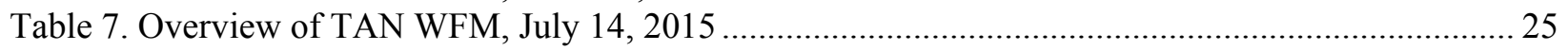

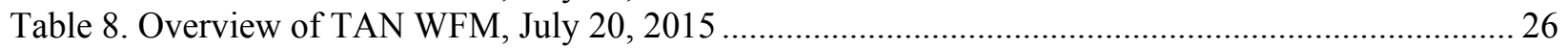

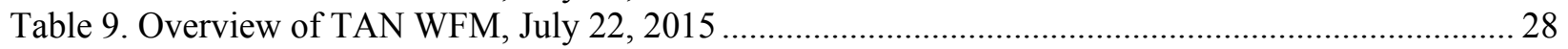

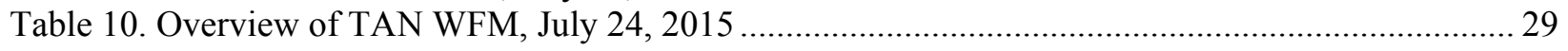

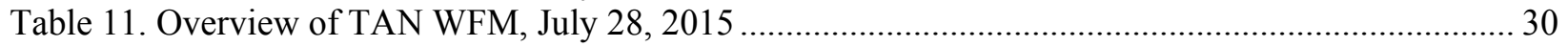

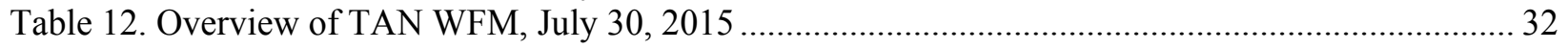

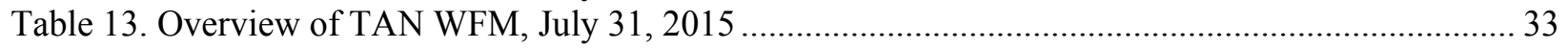

Table 14. Site-Specific Estimates of Total Refrigeration DR Resource, Rebound, and Load-Add

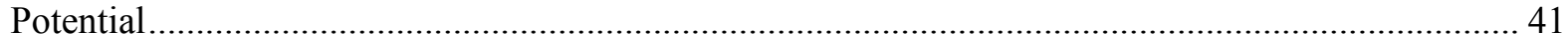

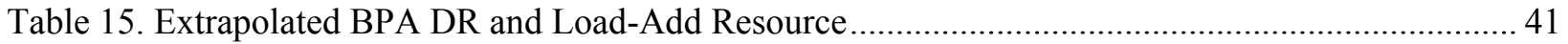




\section{Introduction}

This report describes a pilot project conducted to gain knowledge about the potential magnitude and cost-effectiveness of demand response (DR) in a commercial building sector that consumes a significant amount of energy and power - commercial food sales - by focusing in particular on this sector's largest load, refrigeration. According to the Northwest Power and Conservation Council, DR is "a voluntary and temporary change in consumers' use of electricity when the power system is stressed" (Northwest Power and Conservation Council 2010). Altering demand profiles offers many advantages to utilities. First and foremost, by reducing peak demand, utilities can avoid building new power plants that are only necessary for meeting critical demand and are either unused or run inefficiently for most of the year. One statistic often cited is that just a 10\% reduction in demand for 60 hours per year can eliminate the need for 50100 -megawatt (MW) power plants (Bienert and Haaser 2011). This can lower the cost to produce electricity, and the savings can be passed on to consumers. Shifting demand away from peak hours can also reduce the need for "rolling blackouts." Lastly, being able to alter demand profiles in response to the available electricity in real time will allow more renewable energy to be integrated into utility portfolios, as the availability of renewable resources such as solar and especially wind is much less uniform and predictable than a power plant's.

Beginning with its Fifth Power Plan (Northwest Power and Conservation Council 2005), the Council began to estimate the potential size of DR reserves in the Pacific Northwest and formulate strategies to encourage their creation. In its Sixth Power Plan (Northwest Power and Conservation Council 2010), the Council identified a need for additional pilot research programs to provide the precise and essential information needed to acquire cost-effective DR resources to help balance the supply and demand of electricity on the grid. Substantial progress has been made by utility providers in the Pacific Northwest toward acquiring DR reserves, primarily in direct control of residential air conditioners and irrigation pumps. Other efforts are being made by utility providers to work with DR aggregators and directly with other large power consumers to create interruptible service contracts.

\subsection{Why Supermarkets?}

Supermarkets potentially offer a substantial DR resource because of their high energy intensity and use patterns. In the 2009 Northwest Energy Efficiency Alliance Commercial Building Stock Assessment (The Cadmus Group, Inc. 2009), the "Grocery" category was found to represent approximately 100 million $\mathrm{ft}^{2}$ or roughly $3 \%$ of total commercial floor area. However, it was found to have the second-highest electricity energy use intensity of all building types, at more than 40 kilowatt-hours/ $/ \mathrm{ft}^{2} /$ year, due to extended operating hours and the continuous operation of commercial refrigeration systems needed to preserve perishable products. Electricity use was found to be high all year but to have a summer peaking behavior. Total electricity use by the sector in 2007 was found to be 4,142,000 megawatt-hours, or $11 \%$ of the total energy consumption by commercial buildings in the Pacific Northwest. When averaged evenly over the year, this energy consumption equates to a power of roughly $470 \mathrm{MW}$.

Supermarket refrigeration has several advantages over other commercial building end uses when considering DR. First, the equipment and controls necessary to execute DR strategies are already present in the refrigeration system and its controller. Modern refrigeration controllers are also enabled to communicate over the Internet. These facts reduce the first cost of implementing DR 
in supermarkets because additional equipment is not required. Refrigerated display cases also have built-in thermal capacitance due to the large mass of cooled or frozen product in the display cases. Therefore, product temperature will rise slowly when refrigeration is temporarily suspended, lagging behind the rise in air temperature and extending the duration over which refrigeration can be paused. The display cases can also potentially be used for thermal storage, initially cooling them below their usual set point and then reducing the system's power draw until the display cases reach some critical threshold for DR termination.

Of course, there are limitations to the amount that cooling can be increased or reduced before product quality is affected. For this project, an upper limit of $15^{\circ} \mathrm{F}$ was placed on lowtemperature cases and walk-ins, and a limit of $-5^{\circ} \mathrm{F}$ was placed on ice-cream cases and walk-ins. System capacity limits prevented case and walk-in temperatures from dropping below $-12^{\circ} \mathrm{F}$. Medium-temperature cases, other than the beer case, were off-limits for set point changes.

Unlike store lighting or heating, ventilating, and air conditioning (HVAC), service need not be curtailed when DR is activated. Ideally, DR in refrigeration systems will be invisible to customers. This study investigates the amount of load shed that can be accomplished while keeping products within temperature boundaries recommended by supermarket owners.

\subsection{Demand Response Strategies}

Many utilities in the United States have enacted programs that fall under the general title of "demand response." These programs attempt to bring electricity demand in line with the available supply through alteration of the demand profile, rather than creation of new power plants or temporal variations in the total amount of electricity generation.

A reduction during peak hours as part of a DR strategy can be accomplished in several ways. These methods can be broken into three categories: incentives for reduction, penalties for peak electricity use, and incentives for long-term changes to demand profiles. The following sections describe each of these strategies.

\subsubsection{Incentives for Short-Term Reduction}

Critical peak rebates: When a utility offers a critical peak rebate program, the customer is paid a previously communicated amount for reductions below expected levels during critical periods. These periods, usually on the order of 100 hours per year, normally occur during weekday summer afternoons. The burden of reducing power use is completely on the customer, and the customer may decide how and if to reduce demand during these periods.

Day-before demand bidding: In a somewhat more complex strategy, utilities may provide customers only a day's notice before an anticipated critical period. Customers are asked to "bid" on the amount of demand they plan to reduce the next day. Financial incentives are usually offered only to large clients, whose accounts reach into the hundreds of kilowatts of demand, or to an aggregation of smaller accounts with a combined demand of the same magnitude as that of the larger clients. Bids are expected to be on the order of at least a $15 \%$ reduction in demand during the critical period. This strategy requires special metering and communication devices, as well as a preexisting contract between the client and energy provider. 
Direct load control: Using a more sophisticated strategy, a utility can actually change the operation of equipment at a client's site remotely during a critical event. This strategy requires equipment to be installed beforehand and is primarily used for small commercial and residential customers. Strategies may include shutting off air conditioners in a community for 5 minutes in a staggered arrangement or increasing thermostat set points slightly. However, this strategy may also include actions such as direct control of agricultural pumping devices and other larger equipment.

Automated DR: The most complex, dynamic, incentive-based DR strategy is automated DR. In this strategy, control systems are installed at the client's site. A signal is sent to the control system from the utility notifying it of the opportunity for a DR event and the offered incentive. The control system uses an algorithm to decide whether or not to participate in the event and how to reduce demand if it decides to participate. The controller then alters the operation of electricity-using devices in a preprogrammed manner that best suits the need of the client.

\subsubsection{Penalties for Peak Use}

An alternative to incentive-based DR programs, in which customers opt in to the program, is pricing-based strategies in which default pricing for customers with certain account characteristics varies during the year to dis-incentivize use during peak and critical periods. The following are several ways to accomplish this.

Critical peak pricing: In the simplest strategy, prices for electricity are raised significantly during critical periods in the summer months. Customers know well in advance what the relatively simple pricing structure will be and may adjust their energy use patterns in response.

Time-of-use rates: In a time-of-use rate strategy, broad blocks of clock time are assigned specific prices. For example, two price tiers may exist_- "peak" and "off-peak." Prices are set well in advance and do not change over long periods of time.

Real-time pricing: An extension of the time-of-use concept is dynamic, real-time pricing. In this strategy, prices of electricity change dynamically based on demand and available supply. This strategy may work well in the future when customers are outfitted with control devices capable of making decisions based on the price of electricity.

Variable peak pricing: Variable peak pricing is a combination of time-of-use and real-time pricing strategies. In this strategy, large blocks of clock time (e.g., a 6-hour "peak" and 18-hour "off-peak" period) are portioned well in advance. The price of electricity during these periods, however, changes based on demand.

\subsubsection{Permanent Load Shifting}

The third category of DR strategies involves permanent or semipermanent shifting of demand to more closely match supply.

Energy storage: This strategy may be executed for very large clients, such as large buildings or campuses, by installing on-site energy storage. This may be in the form of thermal storage, battery storage, flow energy storage (pumping during off-peak times), compressed air, or other storage mechanisms. 
Permanent change in use patterns: Smaller clients or clients who do not wish to invest in storage devices may also participate in permanent load shifting through a permanent change in operation. Examples include precooling buildings during the summer before peak hours and charging battery-powered forklifts during off-peak hours. Many utilities provide a payment to clients willing to change their use patterns in this way. Clients are then penalized or their payment is rescinded if agreed-upon changes are not enacted fully.

\subsection{Previous Work}

Relevant, previous DR work includes pilot projects and deployment efforts in supermarkets and in refrigerated facilities. The California Energy Commission reported 7.5 MW of peak demand shed from 300 Albertsons supermarkets (equivalent to 6\% load shed) using sales floor lighting, anti-condensate heaters, and refrigerated display case lighting (California Energy Commission 2005). A single Albertsons refrigerated distribution center in the Pacific Northwest participating in Portland General Electric's Energy Partner DR program reported $200 \mathrm{~kW}$ of load that could be curtailed by changing refrigeration system set points (Portland General Electric 2013). Previous supermarket DR work appears to have excluded the compressors. To our knowledge, Technology Innovation Project \#307 is the first publicly documented DR pilot project in supermarkets that involves the refrigeration systems. 


\section{Scope and Objectives}

This project was concerned with demonstrating DR using the refrigeration system in a real store and quantifying the DR potential inherent in supermarket refrigeration systems. Ancillary aims of the project were to identify practical barriers to implementation of DR programs in supermarkets through real-world tests and to determine which high-level control strategies were most appropriate for achieving certain DR objectives through modeling and field testing. The scope of this project does not include detailed control strategy development for DR or development of a strategy for regional implementation of DR in supermarkets.

Specifically, five objectives were pursued in this study:

1. Conduct pilot evaluation tests to understand stakeholder concerns and barriers to implementing DR.

2. In the course of these tests, evaluate strategies that can provide 3- to 4-hour capacity reserves (identified as a key DR resource by BPA).

3. Conduct additional experiments in a real supermarket for the purpose of developing reliable models of the type needed to quantify DR potential.

4. Use these models to estimate the total aggregated supermarket DR resource available to BPA.

5. Recommend future research and other work that must be done to maximize DR potential in supermarkets. 


\section{Test Supermarket}

The results presented in this report are from the Tanasbourne Whole Foods Market (WFM) site, located in Hillsboro, Oregon, referred to as TAN from here forward. TAN is a 45,591- $\mathrm{ft}^{2}$ store, located 11 miles northwest of downtown Portland. The store operates from 8 a.m. to 10 p.m. Monday through Friday. In order to give the reader a sense of the scale of the possible load shifting opportunity, a graph of the maximum whole building power consumed by the store, from utility bill data for 2 years, is provided in Figure 1.

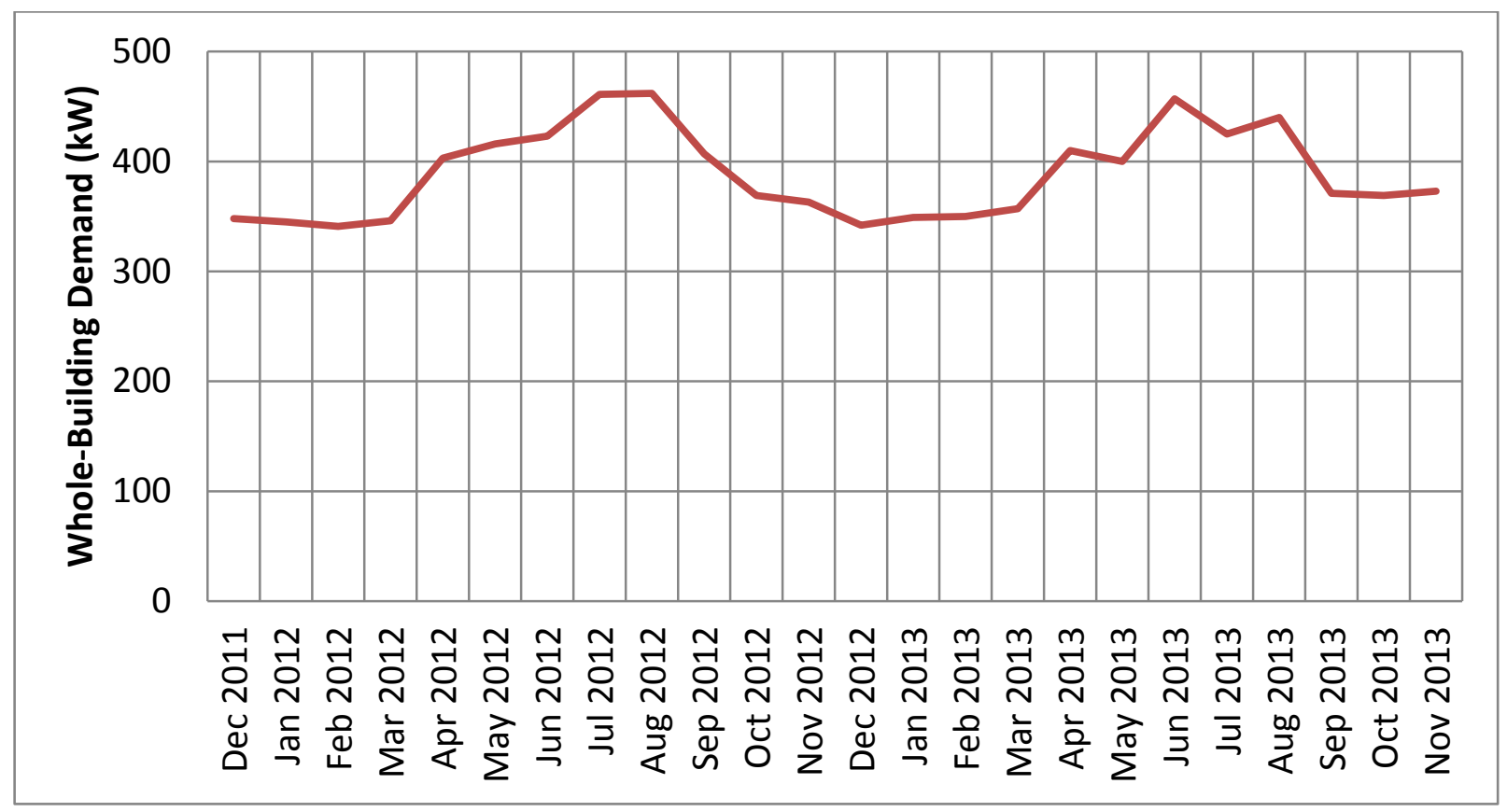

Figure 1. Peak demand data for WFM TAN store

\subsection{Refrigeration Equipment and Controls}

The TAN refrigeration system is divided into two systems, designated by the compressor rack by which they are served. Compressor racks for two suction groups $\left(-20^{\circ} \mathrm{F}\right.$ and $\left.+13^{\circ} \mathrm{F}\right)$ are submetered together in aggregate and called "Rack A" at the store. The other rack- "Rack B"serves $\mathrm{a}+18^{\circ} \mathrm{F}$ suction group only, providing most of the store's medium-temperature refrigeration needs, including most of the open cases. Rack B, which was not available for the purpose of this study, is $90 \%$ larger than Rack A in terms of capacity (Rack B accounts for $65 \%$ of total refrigeration capacity). In fact, all medium-temperature cases were off limits for set point changes at TAN, because medium-temperature product cannot be cooled below normal temperatures without freezing. Additionally, temperature increases for products stored in medium-temperature cases were perceived as risky by the store owner. Thus, DR tests were limited to only low-temperature cases, excluding ice-cream cases.

It is likely that any DR pilot testing program will find similar barriers in any supermarket. However, for an actual DR program, the team believes medium-temperature cases have the potential to provide additional load shedding in real operation. The team believes this load shedding can be accomplished in medium-temperature cases either through secondary loops charged during off-peak, rapid cycling that reduces power while maintaining product 
temperature, the use of phase-change materials, or other means. However, testing these strategies will almost certainly need to be done in a laboratory setting before store owners are comfortable demonstrating them in a real store. This is a major limitation of this study, discussed further in later sections.

A list of the circuits on the low-temperature rack (Rack A) at TAN and the subset of those circuits eligible for DR is included in Table 1. The total capacity of the DR-eligible circuits is roughly $36 \%$ of the total capacity of the single rack $(103,000$ out of 269,000 British thermal units $[\mathrm{Btu}] / \mathrm{h})$.

Table 1. TAN Low-Temperature Refrigeration System Circuits

\begin{tabular}{|c|c|c|c|c|c|}
\hline $\begin{array}{l}\text { Refrigeration } \\
\text { Circuit }\end{array}$ & $\begin{array}{c}\text { DR } \\
\text { Eligible }\end{array}$ & $\begin{array}{c}\text { Capacity } \\
\text { (Btu/h) }\end{array}$ & $\begin{array}{c}\text { Suction } \\
\text { Temperature } \\
\left({ }^{\circ} \mathrm{F}\right)\end{array}$ & Defrost Schedule & $\begin{array}{l}\text { Case Set } \\
\text { Point }\left({ }^{\circ} \mathrm{F}\right)\end{array}$ \\
\hline A02 WI SEAFOOD & Yes & 10,000 & -25 & 4/day $2: 30,8: 30,14: 30,20: 30$ & 0 \\
\hline A03 WI GROCERY & Yes & 24,000 & -25 & 4/day $3: 00,9: 00,15: 00,21: 00$ & -8 \\
\hline A04 WI MEAT FZ & Yes & 9,000 & -25 & $4 /$ day $3: 30,9: 30,15: 30,21: 30$ & 0 \\
\hline A05 FZN MEAT & Yes & 8,960 & -20 & 1/day 4:00 & 0 \\
\hline A06 RI FZ FOOD/IC & No & 15,840 & -16 & 1/day 5:00 & 8 \\
\hline A07 RI ICE CREAM & No & 10,560 & -16 & 1/day $5: 30$ & 8 \\
\hline A08 RI FZ FOOD & Yes & 14,520 & -16 & 1/day 0:00 & 0 \\
\hline A09 RI FZ FOOD & Yes & 11,880 & -16 & $2 /$ day $0: 00,12: 00$ & \\
\hline A10 RI SEAFD & Yes & 10,560 & -16 & 1/day 1:00 & 0 \\
\hline A12 WI BAKERY & No & 15,000 & -25 & 4/day 3:00, 9:00, 15:00, 21:00 & 0 \\
\hline A13 WI PREP FD & Yes & 14,000 & -25 & $3 /$ day $3: 30,11: 30,19: 30$ & -12 \\
\hline A17 ROLL PROD & No & 23,820 & +21 & $6 /$ day $4: 30,8: 30,12: 30$, etc. & +32 \\
\hline A18 WI SEAFOOD & No & 17,500 & +20 & $3 /$ day $5: 00,13: 00,21: 00$ & +39 \\
\hline A19 WI MEAT & No & 17,500 & +20 & $3 /$ day $5: 30,13: 30,21: 30$ & +30 \\
\hline A20 POULTRY \#1 & No & 15,500 & +20 & 4/day 0:00, $6: 00,12: 00,18: 00$ & +36 \\
\hline A21 WI BAKERY & No & 14,500 & +20 & $2 /$ day $00: 30,12: 30$ & +35 \\
\hline $\begin{array}{l}\text { A22 WI PREP } \\
\text { FOODS COOLER }\end{array}$ & No & 26,000 & +20 & 3/day 1:00, 9:00, 17:00 & +34 \\
\hline A23 POULTRY \#2 & No & 9,400 & +20 & 4/day $1: 30,7: 30,13: 30,19: 30$ & +32 \\
\hline
\end{tabular}

The refrigeration system at TAN uses a control strategy commonly known as "floating suction" in which the suction pressure (the pressure of the refrigerant entering the compressors) set point is raised under low load conditions (that is, when less refrigeration is required) such as what occurs at night when customers are not opening reach-in display case doors. The floating suction control strategy saves energy by reducing the temperature "lift" that must be delivered by the refrigeration system to move heat from a cold location (the evaporator) to a warm location (the location of the refrigeration condenser). This control strategy works by shifting the saturated 
suction pressure set point higher when the critical cases are below a specified threshold and lower when the critical cases warm up above that threshold, within a prespecified range. For the low-temperature system at TAN, the critical cases were the ice-cream cases, as ice-cream quality is more sensitive to changes in temperature, and the suction pressure floated between 17 and 27 psig, corresponding to a range of $-7^{\circ} \mathrm{F}$ to $+5^{\circ} \mathrm{F}$ in critical case temperature.

\subsection{Modifications Made for Testing}

Several modifications and additions were made to TAN to allow testing to be done. These are described below.

\subsubsection{Electrical Submeter Installation and Commissioning}

Electrical submetering systems were installed in TAN in June 2014 and commissioned in December 2014. The electrical power measurements were made with revenue-grade SRME2 power monitors for the main service and ERM2 for other monitoring points, and included voltage, current, power factor, kilowatts, kilovolt-amps, and kilowatt-hours.

Table 2. Electrical Submetering Points

\begin{tabular}{lllll}
\hline Store & Circuit Name & $\begin{array}{c}\text { Connection } \\
\text { Type }\end{array}$ & Group & \multicolumn{1}{c}{ Critical Loads } \\
\hline TAN & Main Feed & SRME2 & Main Feed & Main Feed \\
\hline TAN & Rack A & ERM2-104-112 & Refrig & Refrigeration \\
TAN & Rack B & ERM2-104-112 & Refrig & Refrigeration \\
TAN & Panel HL-1 & ERM2-104-112 & Lighting & Lighting \\
TAN & Panel SMDP-1 & ERM2-104-112 & Lighting & Refrigeration Lighting \\
TAN & HVAC-1 & ERM2-104-112 & HVAC & HVAC Unit 1 \\
TAN & HVAC-2 & ERM2-104-112 & HVAC & HVAC Unit 2 \\
\hline
\end{tabular}

Environmental sensors were also installed to measure outdoor and ambient store temperature and humidity. The temperature measurements were made with PT1000 sensors, with a temperature range of $-58^{\circ} \mathrm{F}$ to $221^{\circ} \mathrm{F}$, a resolution of $0.15^{\circ} \mathrm{F}$, and an accuracy of $1^{\circ} \mathrm{F}$ or $0.45 \%$ full span. The indoor sensor was installed on a column on the sales floor between the two frozen food line ups at the end cap of the reach-in frozen food display. The outdoor sensor was installed on a northfacing wall, 6 feet above roof level and away from any artificial heat sources.

\subsubsection{Electronic Evaporator Pressure Regulators}

Testing required electronic evaporator pressure regulators (EEPRs) to be installed on the circuits of interest. The EEPRs allowed each case evaporator to be changed using a digital signal rather than manual adjustment. This allowed precise and quick modulation of set points during testing. It also allowed many tests to be run remotely and on short notice by avoiding the need for a refrigeration technician to be present in the compressor room throughout the entire test. EEPRs were added at TAN on June 7, 2014. 


\subsubsection{Variable-Capacity Compressors}

This project sparked a conversation between WFM store operators and the refrigeration system service manager about adding variable-capacity control to the refrigeration system. Because adding such capability to the lead compressor would allow tighter control and have a positive effect on energy efficiency and DR potential, variable-speed control was added to the lead compressor on April 17, 2015. Digital unloaders were installed on the three lead compressors for each suction group. These allowed for continuous capacity modulation between $50 \%$ and $100 \%$.

\subsubsection{Product Simulators}

In order to control the refrigeration systems based on a simulated product temperature, rather than the relatively quickly changing case air temperature, product simulators were installed on July 21, 2014. The product simulators included a thermistor that was enclosed in a stainless-steel housing using epoxy. The associated thermal mass allowed the product simulators to respond to case air temperature changes in a manner similar to real products. Ten Emerson 508-910x product simulators featuring a $10 \mathrm{k} \Omega$ thermistor with a stated error of less than $0.72^{\circ} \mathrm{F}$ from $40^{\circ} \mathrm{F}$ to $248^{\circ} \mathrm{F}$ were installed inconspicuously in the refrigerated display cases and connected to the analog inputs on a controller unit. Each product simulator contains thermal mass to mimic the thermal behavior of the refrigerated products. The three components are shown together in Figure 2.

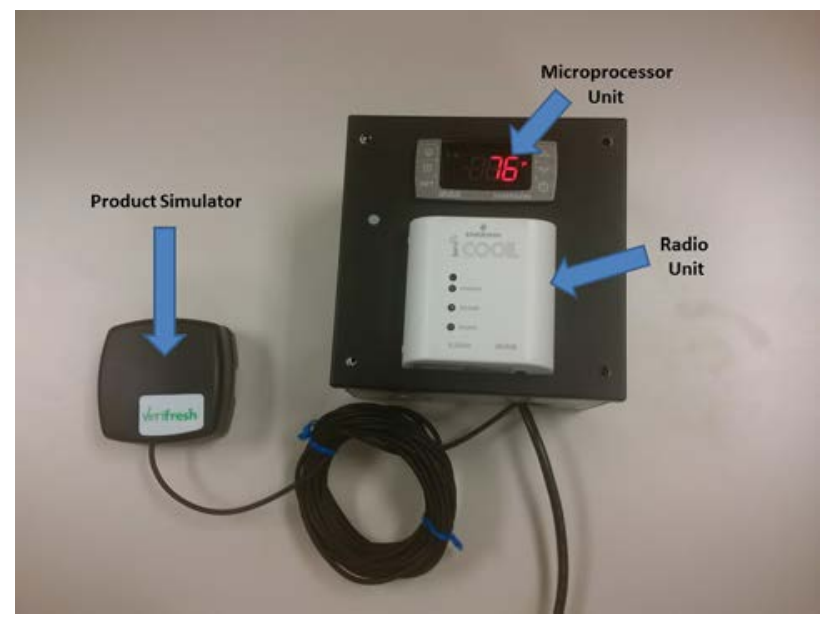

Figure 2. Photo of the product simulator, controller, and radio unit

Controllers specially equipped to allow control based on simulator temperature were also installed on cases of interest. Each controller included (1) a XR75CX-Case Display, which is a microprocessor-based controller that can accept four analog inputs and two digital inputs and communicates via RS-485, and (2) a XJ200 radio module, designed to take the RS-485 signal from the XR75CX and transmit it via radio frequency for up to 200 meters along a clear line of sight, avoiding the need for extensive wiring across the sales floor. 


\section{Methodology}

This section describes the protocols used to test DR algorithms at TAN and to develop a calibrated refrigeration model in order to generalize and extrapolate the results of the pilot testing to other stores, regions, and weather conditions.

\subsection{Critical Limit Testing}

An initial set of tests were performed before any evaluation tests or experiments began. To understand the approximate warming rate of product and case air during a DR event, several tests were run at TAN in which the refrigerant flow was shut off and the resulting case and air temperatures monitored. The rate of product simulator temperature rise in a reach-in frozen food case when refrigeration was turned off, based on an average of three product simulators, was found to be $0.16^{\circ} \mathrm{F} / \mathrm{min}$ or $9.6^{\circ} \mathrm{F} / \mathrm{h}$ (Figure 3 ). The rate of product simulator temperature rise in the meat walk-in freezer when refrigeration was turned off was measured to be roughly $0.04^{\circ} \mathrm{F} / \mathrm{min}$, or $2.4^{\circ} \mathrm{F} / \mathrm{h}$ (Figure 4). Note that the precision of the product simulators was $1^{\circ} \mathrm{F}$, causing the illusion of a stepped progression of temperature in time.

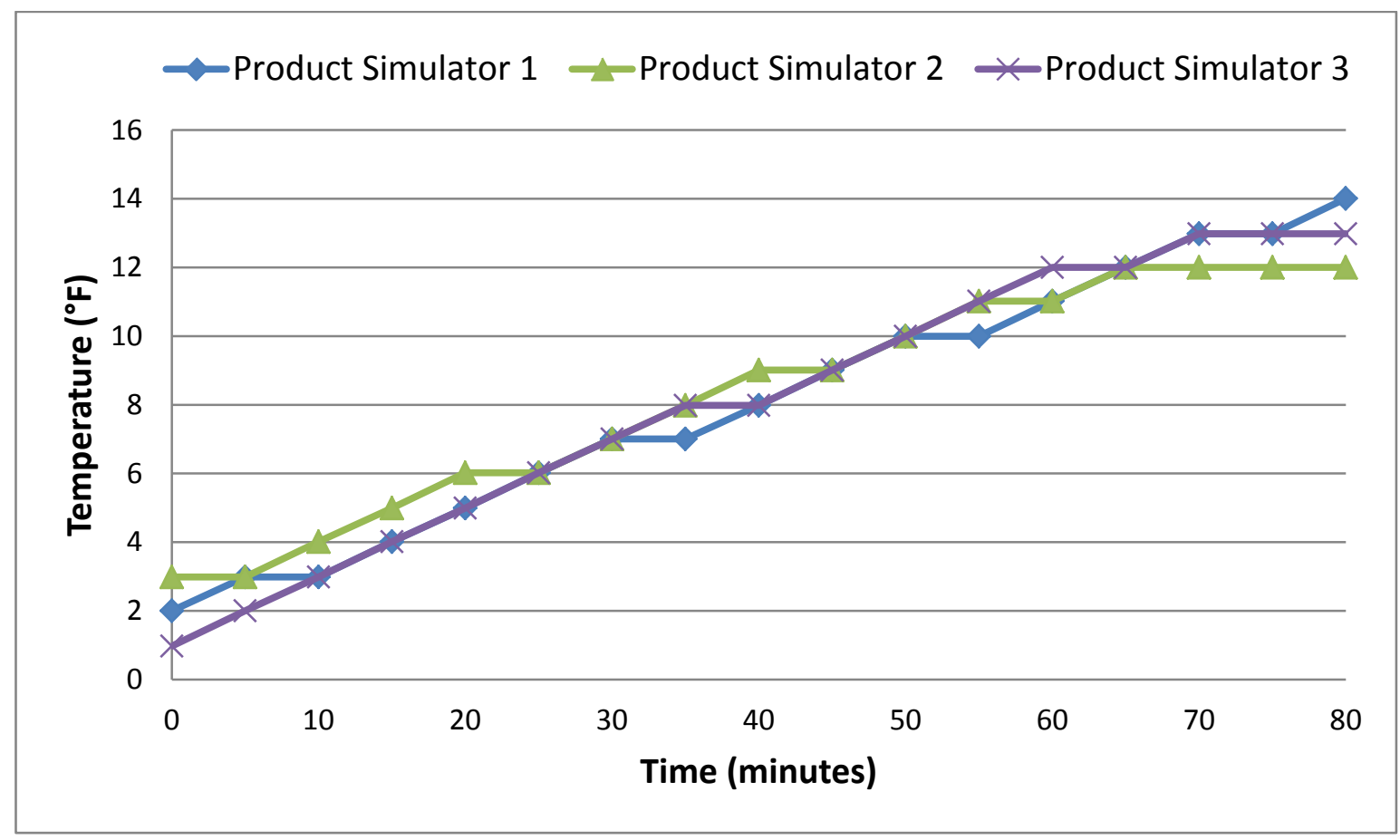

Figure 3. Reach-in frozen food freezer product simulator temperature rise with time 


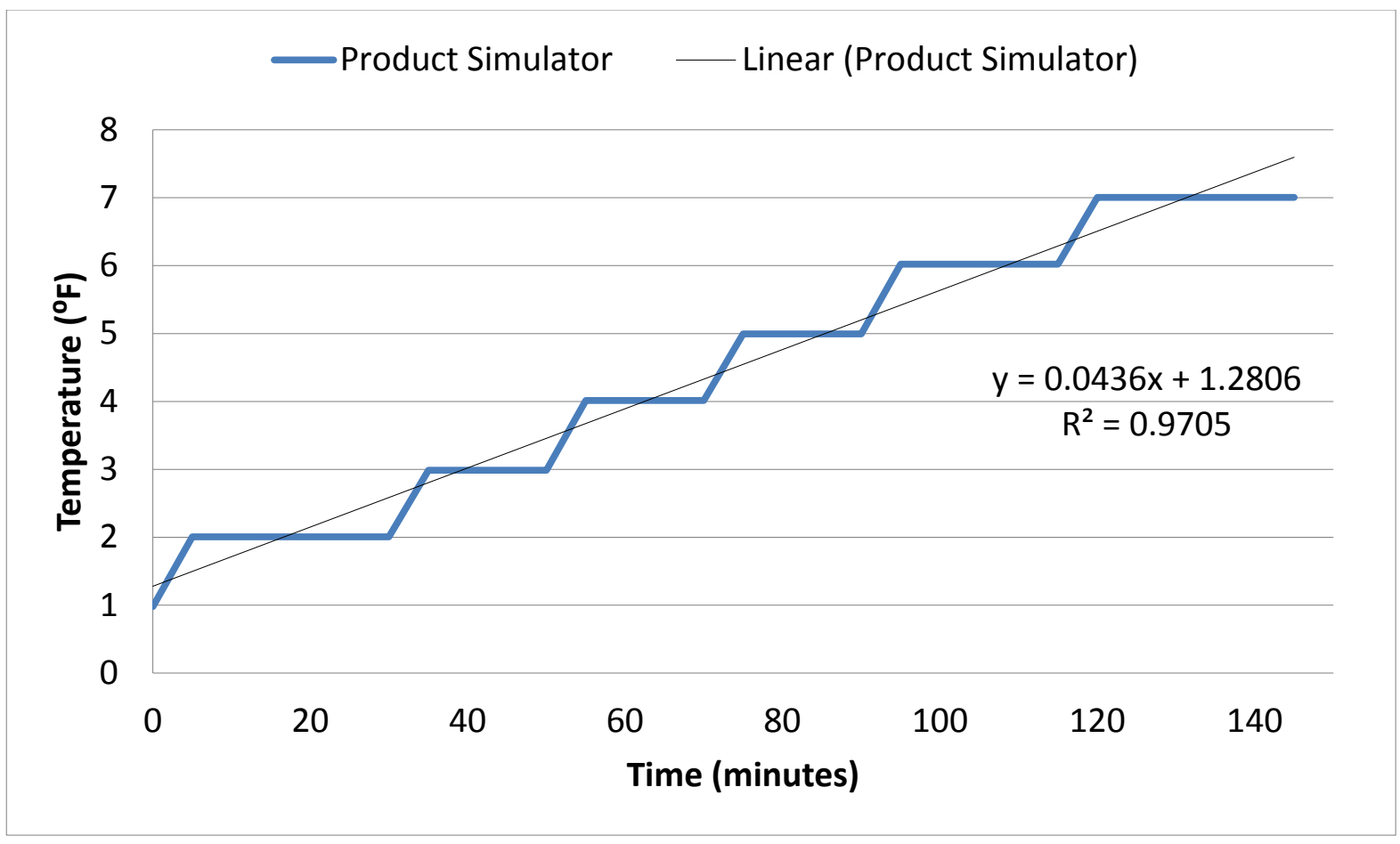

Figure 4. Meat walk-in freezer product simulator temperature rise with time

These numbers set a fundamental time constant for DR. When considering DR, it is appealing to consider shedding the maximum amount of power possible. This goal can be achieved, in theory, by completely turning off the compressor rack. However, how long this strategy can be applied depends on how much products are allowed to warm and the rate of warming. For example, the operation of the compressor rack is typically controlled based on the temperature in so-called "critical cases"-generally reach-in freezer cases containing ice cream. Ice cream cannot exceed $-5^{\circ} \mathrm{F}$ without risking melting, and the product is typically kept at $-8^{\circ} \mathrm{F}$, so the amount of time the system can be "turned off" is only on the order of 20 minutes. This duration can be extended to about 45 minutes if the product is precooled to $-12^{\circ} \mathrm{F}$. In other words, this strategy will not be available to provide longer-term capacity reserves. Another consideration is how to achieve the power reduction - typically supermarket owners and refrigeration technicians are uncomfortable with actually turning compressors off, because it is disruptive to the system operation and interferes with their ability to respond to refrigeration needs.

Instead, it is necessary to consider strategies that shed less power but can be maintained for a longer time. The main focus of this report is evaluating strategies using existing equipment and controls that can provide 3- to 4-hour capacity reserves, which were identified as a key DR resource by BPA.

\subsection{Demand Response Evaluation Tests}

The following sections describe the various methods used during the DR evaluation test phase of the project. 


\subsubsection{Demand Response Using Existing Control Points}

Without adding equipment or making significant control algorithm changes, there were two control strategies at TAN that the team could use to modify the power consumption of the lowtemperature refrigeration system. These were the suction pressure set point of the whole compressor rack and the evaporator discharge air temperature (DAT) set points for the individual noncritical (i.e., not containing ice cream) reach-in and walk-in refrigeration circuits. Figure 5 shows the configuration of a typical refrigeration system, including the monitoring and control points most relevant to this study, indicated with green lines.

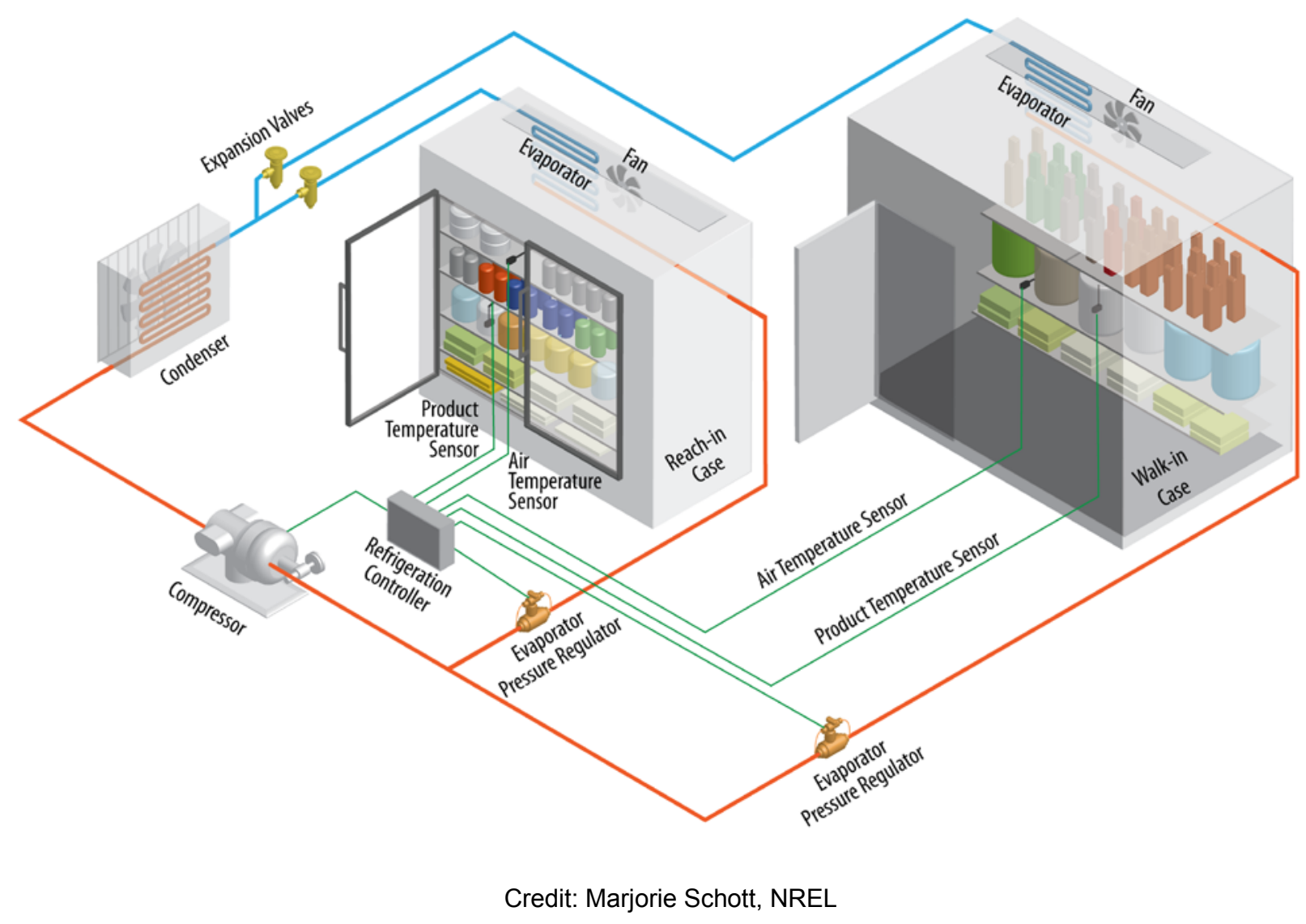

Figure 5. Illustration showing typical refrigeration system configuration

The saturated suction pressure set point could only be made $4^{\circ} \mathrm{F}$ colder $\left(-8^{\circ} \mathrm{F}\right.$ to $\left.-12^{\circ} \mathrm{F}\right)$, reflecting the capacity of the system, and $3^{\circ} \mathrm{F}$ warmer (from $-8^{\circ} \mathrm{F}$ to $-5^{\circ} \mathrm{F}$ ) due to concerns about melting the ice cream in the critical reach-in case. The evaporator DAT set points were more flexible and could be varied from $-12^{\circ} \mathrm{F}$ to $+15^{\circ} \mathrm{F}$. For all product types, it is critical to understand the supermarket owner's concerns in terms of food safety, food integrity, and store operation, so that DR does not result in adverse outcomes.

\subsubsection{Demand Response Using Product Simulators}

Initially, product temperature simulators were installed in reach-in and walk-in cases at TAN as a risk mitigation strategy. The product simulators have a thermal mass that mimics a frozen product and a central thermistor that sends data to the refrigeration controller for viewing and 
trending. It was thought that by monitoring a proxy for the product temperature, the team would better avoid testing protocols that risked exposing frozen products to unacceptably high temperatures. However, the project team quickly noticed that the product simulators installed at TAN presented an opportunity for a DR strategy that leveraged the thermal mass of frozen products.

Because reach-in refrigerated display cases are not well insulated and are subject to infiltration of sales floor air when the doors are opened, the air temperature in critical cases that contain ice cream rises quickly when the compressor rack turns off. This limits the time that compressors can be completely turned off before the compressor rack must ramp back up to keep the case cold. However, the frozen products warm up much more slowly because of their thermal mass. Therefore, if the product simulators are used to control the refrigeration system and the set point of the critical cases is raised, the amount of time that refrigeration compressors can be turned off to achieve DR can be substantially increased.

Following the DR period, the team found that control must be returned back to the evaporator DAT. Otherwise, the compressors ramp up to high power for an extended period of time trying to bring the product simulator temperature down. Under DAT control, the air temperature control signal will drop quickly, allowing the system to return to its original operation (and power consumption) sooner. Product temperature eventually returns to its normal level but without causing the compressors to run at full capacity for an extended period of time.

The challenge with a DR strategy controlled from the product simulator temperature is that it is easy for the product temperature to overshoot its set point due to thermal inertia. In other words, the compressors will only ramp up once the product simulator temperature has exceeded the set point, but the product simulator temperature will continue to rise for some finite amount of time. Therefore the set point must be set conservatively, well below the threshold where melting is considered likely, or considerable additional work must be done to ensure that overshoots do not result in melted product. These considerations add risk and therefore reduce the likelihood of adoption by supermarket owners relative to more standard DR strategies that leverage DAT set points, regardless of the DR advantages.

\subsubsection{Precooling}

DR using supermarket refrigeration systems depends on cooling energy stored in the frozen products, so it is logical to think that it should be possible to "precharge" thermal storage before the DR period to achieve a larger response. This precooling can be achieved by dropping the discharge air set point for the critical cases and noncritical reach-ins and walk-ins down to $-12^{\circ} \mathrm{F}$ for several hours. This was done operationally by dropping the set point for the critical cases and opening the EEPR valves on the noncritical cases and walk-ins. As a result, the entire system started out the DR period with a temperature of approximately $-12^{\circ} \mathrm{F}$. Clearly, this strategy is feasible only if the supermarket has been given enough advance warning of the need for DR resources. The impact of precooling on overall energy use is likely to be site-specific, but was not investigated as part of this study. However, shifting the demand peak earlier in the day by lowering the rack temperature (without then raising set points) is one way to provide a demand-add response, consuming additional power to balance a variable renewable resource such as wind, for example. 


\subsection{Modeling Methodology}

Pilot tests offer many opportunities for identifying real-world issues that may arise during DR events, including a better understanding of the potential load shedding available in supermarkets, and insight into the effectiveness of specific strategies. However, due to the time and cost constraints associated with field testing, a much broader understanding of DR potential as well a more exhaustive investigation of candidate control strategies can be gained by using accurate and reliable models.

To that end, two modeling efforts were pursued for this project. The first was the best effort possible with existing building energy simulations software, in this case EnergyPlus (U.S. Department of Energy 2015). For the second effort, new models were generated from measured data that allowed for simulation of refrigerated case dynamics. As far as the team knows, these models are the first of their kind.

\subsubsection{EnergyPlus Modeling}

The analysis of DR resources began with a calibrated EnergyPlus model of a WFM storesimilar, but not identical — to the stores the team worked with near Portland, Oregon. It was originally believed that DR would be able to take advantage of ice cream and some mediumtemperature cases. To this end, the team modeled a DR scenario in which the team shut off all cases/walk-ins on one low-temperature rack and select cases/walk-ins on the mediumtemperature rack. The low-temperature cases and walk-ins that were disabled included the icecream cases, two bakery dessert cases, the blast chiller, and the bakery and kitchen freezer. The medium-temperature cases that were disabled included produce/floral cases/coolers, and cases with nondairy drinks (soda, beer, etc.).

This analysis calculated that it was possible to shed roughly $60 \mathrm{~kW}$ for the duration of a 4-hour DR event. Because of constraints within EnergyPlus, the effect of this event on product temperature was not known. This model was a good place to start, because it was a detailed numerical model of a real supermarket and EnergyPlus is designed to capture the power consumption of the refrigeration system.

At the same time, the approach was limited because refrigeration modeling in EnergyPlus is designed to capture steady-state operation and is load-based, meaning that EnergyPlus captures the energy flows in and out of the refrigeration equipment. However, unlike HVAC equipment, it does not model refrigerated case air or product temperatures. Therefore, typical control strategies that might be used to deliver DR cannot be realistically modeled and the impact on food temperatures - a key consideration for refrigeration DR - could not be evaluated.

Therefore, the EnergyPlus DR analysis represented an upper bound on the DR resource available. The team now knows from pilot testing that a 4-hour DR event with no precooling and no supplied refrigeration for a reach-in frozen food case is simply unrealistic - the product will get too warm. These dynamics can be captured with a different modeling approach, however, described below in Section 4.3.2. 


\subsubsection{Gray-Box Modeling}

As mentioned above, the current state of supermarket and refrigeration modeling is inadequate for DR studies for a few reasons. First, available models such as those in EnergyPlus and elsewhere are exclusively steady state. Refrigerated display cases are assumed to run at a constant operating temperature, and any changes in this temperature are accomplished via inputs from the model operator and assumed to occur instantaneously. This type of model does not allow information to be extracted that may be used to make decisions about or assess the potential of DR control strategies. Models to be used for this purpose must consider case temperatures, including air and/or product and possibly refrigerant state, as a dynamic variable that reacts to boundary conditions such as set points, ambient store conditions, and outdoor conditions.

Second, currently available models do not explicitly model product temperature. Product temperature is either an input or assumed to be a simple function of the "case temperature," which is an input. Information about how a product reacts to changes in system operation during a DR event cannot be obtained from such models.

Lastly, available models assume an isothermal environment within the case. As in-store testing shown below indicates, product temperatures may vary by as much as $4^{\circ} \mathrm{F}$ at times. Therefore, more refined models that include either explicit modeling of extreme temperatures within the case or a compensation for this variation must be developed.

In order to provide models that may be used by designers, store operators, and utilities to assess DR potential and plan for system operation during DR events, the team developed new models of refrigerated display cases and walk-ins. For the display case modeling efforts, the team followed the approach of O'Connell et al. (2015). This approach is particularly well suited for modeling problems in which the transient variation of model variables is of interest, and in which a simplified approach must be taken to reduce the computation time required for model execution. The approach also lends itself particularly well to thermal modeling problems, as the analogy between heat transfer in a thermal system and electrical circuits is well documented and has a well-accepted theoretical underpinning.

For the purposes of this project, models were developed with a structure that would allow us to capture important DR response information while allowing for many model runs through a simplified model structure and efficient solution algorithm. To this end, several assumptions were made:

- The time scale of the process in the refrigeration loop (compression, condensing, expansion, and evaporation) is much smaller than that of the time scale for the temperature changes in the refrigerated product. This assumption is justified by experiments conducted in this project. For this reason, modifications to evaporator temperature and condensing temperature are assumed to be instantaneous, while the temperatures in the display cases (air temperature, product temperatures, and intermediate temperature such as interior and exterior wall temperatures) are assumed to change continuously in time. The dynamics of these latter variables are captured explicitly in the models. 
- A semiempirical correlation between saturated suction temperature (SST), saturated condensing temperature, cooling load delivered, and power input to the compressors is assumed. The correlation is semiempirical in that it outputs the necessary power input as a function of actual load on the system and Carnot coefficient of performance (COP). Carnot COP is the theoretical relationship between input power and cooling load delivered. A literature review shows that using the Carnot COP multiplied by a modification factor that aggregates all the inefficiencies in the system provides acceptable predictions of necessary power at the range of operating conditions expected to be seen in this study (Hasse et al. 1996, 10-18). The model employed is:

$$
\dot{Q}_{\text {cooling }}=\alpha * P_{\text {input }} * C O P_{\text {Carnot }}=\alpha * P_{\text {input }} *[S S T /(S C T-S S T)]
$$

Where:

$\dot{Q}_{\text {cooling }}$ is the rate of energy removal from the system $[\mathrm{kW}]$

$P_{\text {input }}$ is the electrical power input into the compressors $[\mathrm{kW}]$

$C O P_{\text {Carnot }}$ is the theoretical maximum efficiency (Carnot efficiency)

SST is the saturated suction temperature $[\mathrm{K}]$

SCT is the saturated condensing temperature $[\mathrm{K}]$

And $\alpha$ is the empirical modification factor.

Hasse et al. (1996, 10-18) use a value of 0.4 for the modification factor and justify this assumption for SST values from $-30^{\circ} \mathrm{C}$ to $-10^{\circ} \mathrm{C}$ (Hasse et al. 1996, 10-18). Data collected by NREL show that a modification factor of 0.38 is appropriate for a real store in Colorado.

\subsubsection{Gray-Box Model Data Generation}

Separate sets of tests were conducted to generate data for gray-box model calibration and validation. Instrumentation of cases and walk-ins of interest, debugging, and testing were conducted over a 2 -week period in July 2015 . One low-temperature $\left(0^{\circ} \mathrm{F}\right.$ steady-state set point $)$ walk-in seafood cooler and one vertical closed frozen-food display case (also $0^{\circ} \mathrm{F}$ steady-state set point) were selected for testing.

The first task was instrumenting the case/walk-in of interest with multiple product simulators in order to capture the range of temperature variation within the case/walk-in. Whereas earlier tests used only one product simulator in each case/walk-in as a replacement for the single air temperature normally used for control, in this phase the team wanted to develop models that captured temperature variation. To this end, the vertical frozen food case was outfitted with six product simulators and the walk-in with three. Product simulators were placed to capture extreme temperatures, remain inconspicuous, and allow uninterrupted use of the case/walk-in. Product locations are shown in Figure 6. 


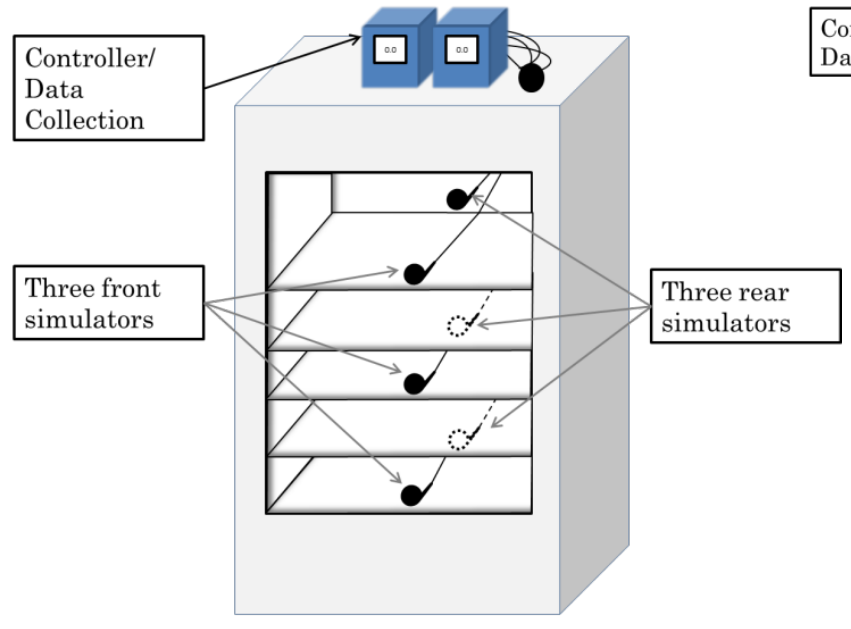

Vertical Closed Frozen Food Case

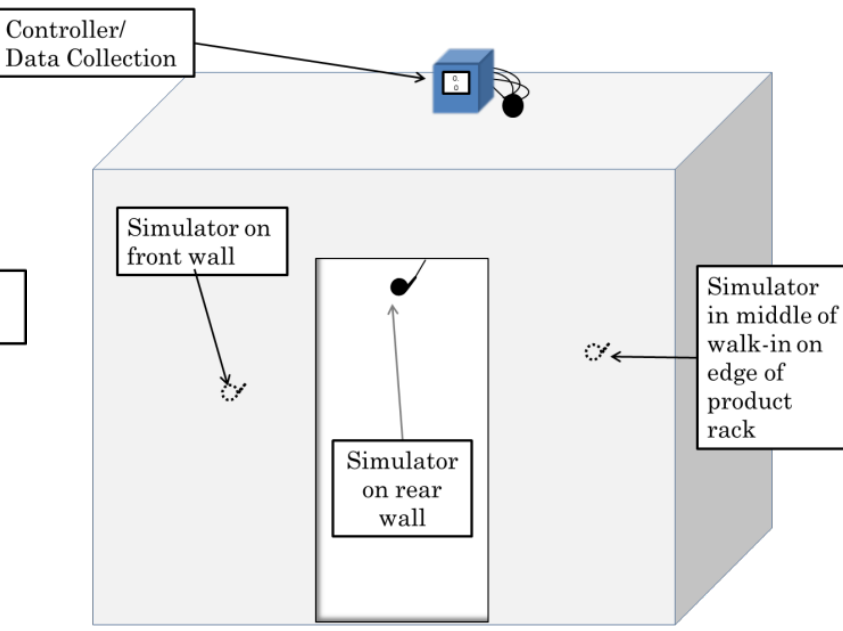

Low Temperature Walk-In

Credit: Jordan Clark, NREL

\section{Figure 6. Product simulator locations}

With the product simulators installed and mapped in the wireless data acquisition system, testing began. The testing conducted for the purposes of model development was of a very specific nature. It was originally thought that model development might be possible with operational data only. However, for the specific type of modeling done in this study, operational data were insufficient. As explained above, gray-box modeling techniques were used for model development because of their ability to provide dynamic models that solve quickly and don't require large amounts of information to be input. However, gray-box models can only be developed from data resulting from a continuously excited system, meaning temperatures in the system are changing throughout the data collection period. Ideally, the system is excited in a random or seemingly random manner during data collection in order to determine that any trends in the dynamics of the outputs of the system were not created by trends in the inputs but by processes within the system that are captured in the model.

For this reason, the system was excited with a pseudo-random binary signal (PRBS). The signal was pseudo-random in that it behaved and appeared as a completely random binary signal, but it was generated from a deterministic algorithm (Godfrey 1980, 527-534). The team manipulated the case/walk-in set point, which oscillated between a high $\left(+15^{\circ} \mathrm{F}\right)$ and low $\left(0^{\circ} \mathrm{F}\right)$ value. This ensured that product temperatures never entered unsafe territory during system excitation, but that the system was sufficiently excited to extract relevant model parameters. An example of the PRBS is given in Figure 7. 


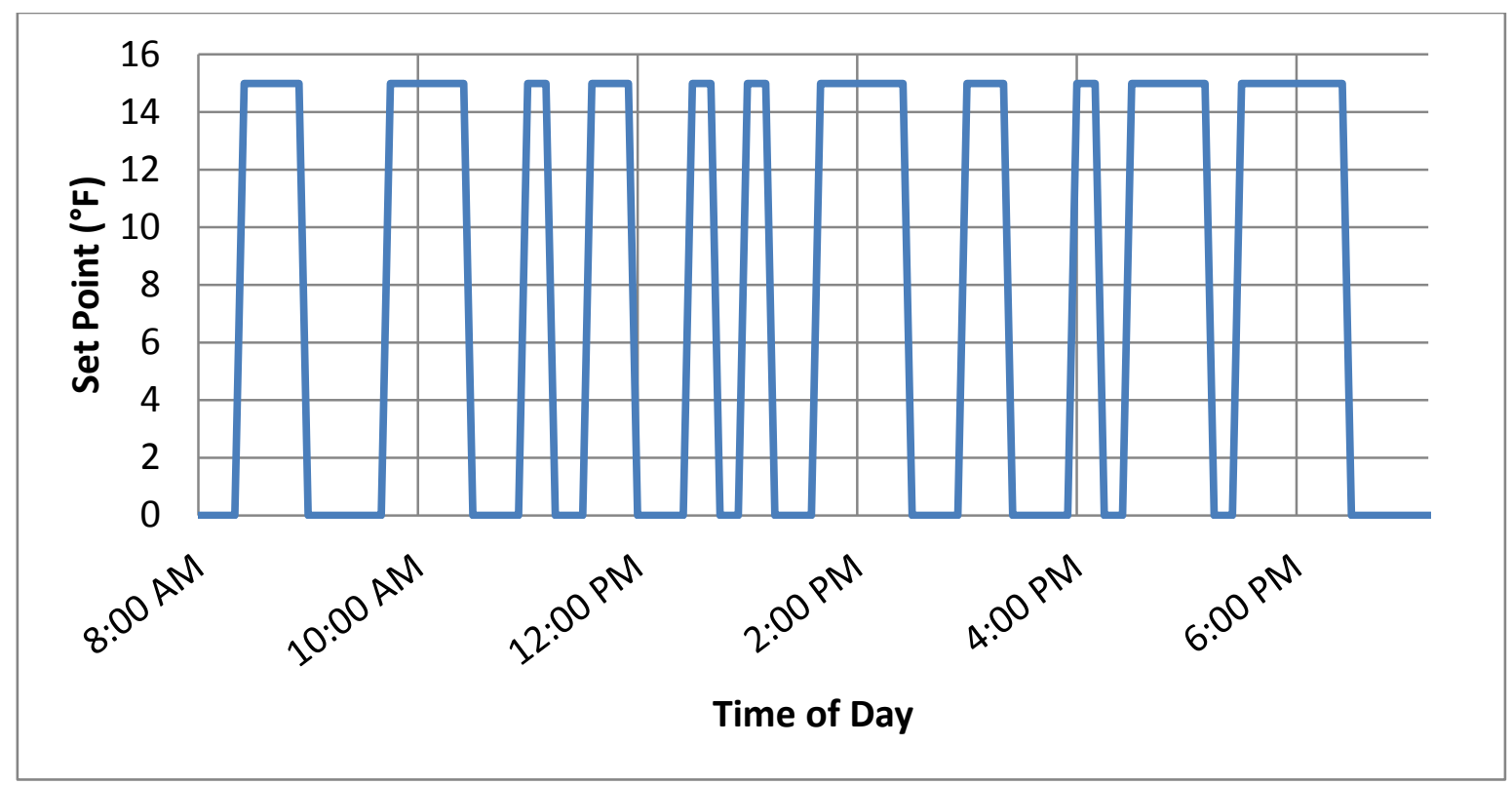

Figure 7. PRBS signal used for training model

Once all data acquisition equipment and control equipment was functioning as needed, two tests were conducted. In both tests, set points in both the walk-in and vertical case were varied with a PRBS over the course of a day. For the training tests, used to identify model parameters, set points were varied over the course of 11 hours; product temperatures continued to respond to the last change in temperature for an additional 3.5 hours. During validation testing, used to determine the goodness of the model developed with the training data, set points were varied over the course of 8.5 hours, and the temperatures continued to respond for an additional 4.5 hours before reaching steady state.

\subsection{Statistical Analysis of Measured Data}

Any energy-saving or power-modifying intervention requires a baseline to serve as a benchmark. The baseline is the energy or power consumption in the absence of the measure. This baseline is theoretical and must be modeled as accurately as possible. There are many approaches to constructing a statistical "customer baseline load" (CBL). One standard approach accounts for time of week and averages the 10 preceding days of data. For example, if 2 p.m. is included in a DR period, data from the previous 10 weekdays are averaged to provide the baseline. This is the approach used by the California Independent System Operator (Goldberg 2013). Ten prior weekdays provide a sample that is likely to have similar weather to the actual DR date.

For systems such as HVAC and refrigeration, accounting for the impact of outdoor air temperature and outdoor dew point variations on power consumption increases the baseline accuracy. These factors can be accounted for using a multiple linear regression statistical model of the CBL based on time of week and meteorological variables and by evaluating uncertainty in the CBL and therefore in the modeled DR. Many buildings will have, at best, only 15-minute "interval" data available from the main electrical meter. A DR will have to contend with the statistical noise inherent in this signal. Isolating the building system or piece of equipment in question using strategically placed electrical submeters will reduce statistical noise. 
To establish the baseline CBL, the team used a method previously devised for a separate BPAfunded project (Technology Innovation Project \#308). In that project, Heaney, Doebber, and Hirsch (2015) determined that a regression model including factors for diurnal variations and weekly variations, outdoor air temperature, and outdoor air dew point provided the best estimate for baseline power consumption during a DR event. Interactions and polynomials did not improve the model. They concluded that their approach meets or exceeds the accuracy of a 10day rolling average.

For more information on the modeling methodology and validation, refer to Heaney, Doebber, and Hirsch (2015). 


\section{Measurements}

Results are presented below for (1) the field tests conducted at TAN, and (2) the training and validation tests performed to calibrate the refrigeration gray-box model. For each test, power data are shown. For gray-box model development measurements, corresponding product and air temperatures are shown as well.

\subsection{Demand Response Tests}

Several tests were conducted at TAN to test various DR methods. These methods included various combinations of local and global control. In order to guide the reader through the many combinations of relevant variables and strategies tested on each day, a summary is included in Tables 3 and 4 . It should be noted that tests were done at various outdoor air temperatures, which affects the amount of load present and thus the amount of load shed available. It should also be noted that some strategies are more suitable for a "day-ahead" DR bid, when the client has the option to do precooling, and some strategies are more likely to be implemented when the client has little notice before a DR event. Types of control tested include:

- Precooling the cases before the simulated DR event (indicated by the "Precool" column in Table 1): This strategy lowers product and air temperatures before the DR event begins to allow a longer period of load shedding during the DR event.

- Changing the DAT set point for noncritical cases: This produces a lower enthalpy change on the low-pressure side of the refrigerant circuit, presumably shedding load.

- Changing the DAT set point for the critical case: This increases the SST for the entire rack, increasing refrigeration efficiency and decreasing the temperature drop in the refrigerant loop, shedding load.

- Controlling the compressor based on product simulator temperature rather than DAT: DAT is normally used to control compressor operation because refrigeration systems usually run at steady state and thus the product temperature and DAT are roughly equal. As mentioned earlier, the product has a much larger thermal mass than the case air, and thus responds more slowly to changes in operation. If compressor control is switched from the air temperature to a product simulator temperature, a longer load shed can be accomplished while still working within the same food safety constraints. 
Table 3. Site and Control Parameters for Each DR Test

\begin{tabular}{llllll}
\hline Test & Date & Precool & $\begin{array}{c}\text { Change } \\
\text { Critical Case } \\
\text { DAT Set } \\
\text { Point }\end{array}$ & $\begin{array}{c}\text { Change } \\
\text { Noncritical } \\
\text { Case DAT Set } \\
\text { Point }\end{array}$ & $\begin{array}{c}\text { DAT or } \\
\text { Product } \\
\text { Simulator } \\
\text { (PS) } \\
\text { Control }\end{array}$ \\
\hline Test \#1 & $9 / 23 / 14$ & No & Yes & No & DAT \\
\hline Test \#2 & $11 / 10 / 14$ & No & Yes & No & PS \\
\hline Test \#3 & $7 / 14 / 15$ & No & Yes & No & DAT \\
\hline Test \#4 & $7 / 20 / 15$ & No & Yes & Yes & DAT \\
Test \#5 & $7 / 22 / 15$ & Yes & Yes & Yes & DAT \\
\hline Test \#6 & $7 / 24 / 15$ & Yes & Yes & No & DAT \\
\hline Test \#7 & $7 / 28 / 15$ & No & No & Yes & DAT \\
\hline Test \#8 & $7 / 30 / 15$ & Yes & Yes & Yes & DAT \\
Test \#9 & $7 / 31 / 15$ & Yes & No & No & DAT \\
\hline
\end{tabular}

Results for each of the DR tests are listed in Table 4. Additional details and results for each test are provided in subsequent sections. It should be noted that for a test to be conducted, several variables needed to align on the same day, namely that the myriad equipment necessary for each test was installed and functioning as desired, and that personnel were available to make changes. Test results are presented chronologically below.

Table 4. DR Results for Each Evaluation Test

\begin{tabular}{lccccccc}
\hline Test & $\begin{array}{c}\text { Load Shed } \\
\text { Range } \\
\mathbf{\pm 9 5 \%}(\mathbf{k W})\end{array}$ & $\begin{array}{c}\text { Average } \\
\text { Load } \\
\text { Shed } \\
\mathbf{( k W )}\end{array}$ & $\begin{array}{c}\text { Outdoor Air } \\
\text { Temp. } \\
\text { Range }\left({ }^{\circ} \mathbf{F}\right)\end{array}$ & $\begin{array}{c}\text { Average } \\
\text { Outdoor } \\
\text { Air } \\
\text { Temp. } \\
\left({ }^{\circ} \mathbf{F}\right)\end{array}$ & $\begin{array}{c}\text { Baseline } \\
\text { Load } \\
\text { Range (kW) }\end{array}$ & $\begin{array}{c}\text { Average } \\
\text { Baseline } \\
\text { Load } \\
\mathbf{( k W )}\end{array}$ & $\begin{array}{c}\text { Average } \\
\text { Additional } \\
\text { Precool Power } \\
\text { Draw (kW) }\end{array}$ \\
\hline Test \#1 & $-0.4-8.0$ & 3.8 & $60.8-63.7$ & 62.3 & $22.5-29.0$ & 26.2 & N/A \\
\hline Test \#2 & $4.7-13.2$ & 9.0 & $59.7-61.7$ & 60.8 & $27.6-30.8$ & 28.9 & N/A \\
\hline Test \#3 & $-1.3-8.3$ & 3.5 & $87.4-92.3$ & 90.2 & $35.2-42.7$ & 38.9 & N/A \\
\hline Test \#4 & $3.2-13.6$ & 8.4 & $90.0-94.3$ & 92.2 & $35.5-44.3$ & 40.6 & N/A \\
\hline Test \#5 & $-1.9-9.2$ & 3.6 & $71.4-75.2$ & 73.8 & $29.1-35.9$ & 32.6 & 7.7 \\
\hline Test \#6 & $-3.3-7.6$ & 2.1 & $84.9-91.4$ & 88.0 & $35.4-42.0$ & 39.2 & 3.5 \\
\hline Test \#7 & $-3.1-6.8$ & 1.9 & $87.1-92.1$ & 89.3 & $35.5-41.8$ & 38.3 & N/A \\
\hline Test \#8 & $2.5-11.9$ & 7.2 & $95.5-111.9$ & 105.8 & $43.3-50.7$ & 47.2 & 4.6 \\
\hline Test \#9 & $-7.3-3.1$ & -2.1 & $104.4-108.5$ & 106.1 & $44.4-49.4$ & 46.7 & 5.7 \\
\hline
\end{tabular}




\subsubsection{Test \#1: Tanasbourne, Sept. 23, 2014}

The purpose of this test was to establish an estimate of the amount of power that could be shed by simply raising the DAT set point of the critical case (ice cream) to the set point of the noncritical case. This change directly affects the saturated suction pressure of the entire rack in a few ways. First, it increases the COP of the system by reducing the temperature drop in the refrigerant loop. Second, it reduces the problem of "wasting" cooling by throttling refrigerant cooled for the critical case for use in noncritical cases. Third, it reduces the overall cooling load on the entire rack.

In this test, the team raised the saturated suction pressure of the entire rack by modifying the set point of the critical case from $-8^{\circ} \mathrm{F}$ to $0^{\circ} \mathrm{F}$, from 8:30-10 a.m. After this hour and a half, the icecream case reached $-5^{\circ} \mathrm{F}$, the upper limit for these cases, and normal operation was resumed. Control of other cases on the same rack was maintained at default settings.

The resulting change in power consumption and other pertinent test results are summarized in Table 5 and Figure 8. It should be noted that this test was done at the beginning of the day, when the store was relatively unoccupied and that the product simulators were not yet installed in the critical case. One would expect temperatures to rise faster at later times in the day when more customers are shopping and opening case doors, curtailing such a DR event more quickly. However, a control based on product simulator temperature rather than case (air) temperature may allow a longer DR event. Both of these hypotheses were examined during subsequent tests.

Table 5. Overview of TAN WFM, Sept. 23, 2014

\begin{tabular}{lc}
\hline Parameter & Value during DR Test \\
\hline Load shed range $\pm 95 \%(\mathrm{~kW})$ & $-0.4-8.0$ \\
\hline Average load shed $(\mathrm{kW})$ & 3.8 \\
\hline Outdoor air temperature range $\left({ }^{\circ} \mathrm{F}\right)$ & $60.8-63.7$ \\
\hline Average outdoor air temperature $\left({ }^{\circ} \mathrm{F}\right)$ & 62.3 \\
\hline Baseline load range $(\mathrm{kW})$ & $22.5-29.0$ \\
Average baseline load $(\mathrm{kW})$ & 26.2 \\
\hline
\end{tabular}




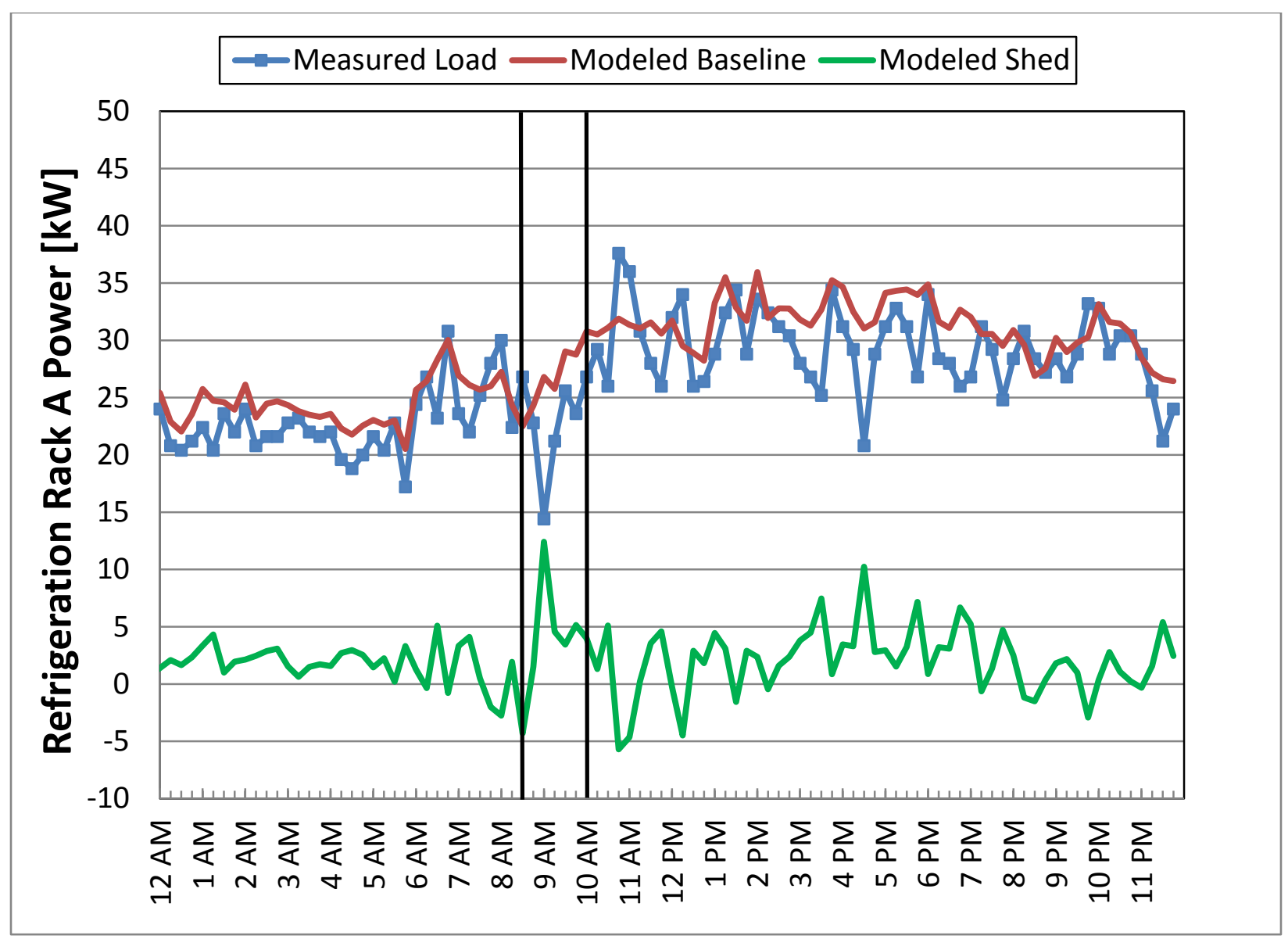

Figure 8. DR event at TAN WFM, Sept. 23, 2014

\subsubsection{Test \#2: Tanasbourne, Nov. 10, 2014}

For this test, control of the critical case was shifted from DAT (the default control variable) to the newly installed product simulator temperature in hopes that a longer DR event could be accomplished. The saturated suction pressure of the entire rack was also changed from $-8^{\circ} \mathrm{F}$ to $-5^{\circ} \mathrm{F}$. The noncritical case and walk-in set points were left at their default values. The DR event ended after only an hour and fifteen minutes when the ice cream reached its maximum temperature. The resulting change in power consumption and other pertinent test results are summarized in Table 6 and Figure 9. 
Table 6. Overview of TAN WFM, Nov. 10, 2014

\begin{tabular}{lc}
\hline Parameter & Value during DR Test \\
\hline Load shed range $\pm 95 \%(\mathrm{~kW})$ & $4.7-13.2$ \\
\hline Average load shed $(\mathrm{kW})$ & 9.0 \\
\hline Outdoor air temperature range $\left({ }^{\circ} \mathrm{F}\right)$ & $59.7-61.7$ \\
\hline Average outdoor air temperature $\left({ }^{\circ} \mathrm{F}\right)$ & 60.8 \\
\hline Baseline load range $(\mathrm{kW})$ & $27.6-30.8$ \\
\hline Average baseline load $(\mathrm{kW})$ & 28.9 \\
\hline
\end{tabular}

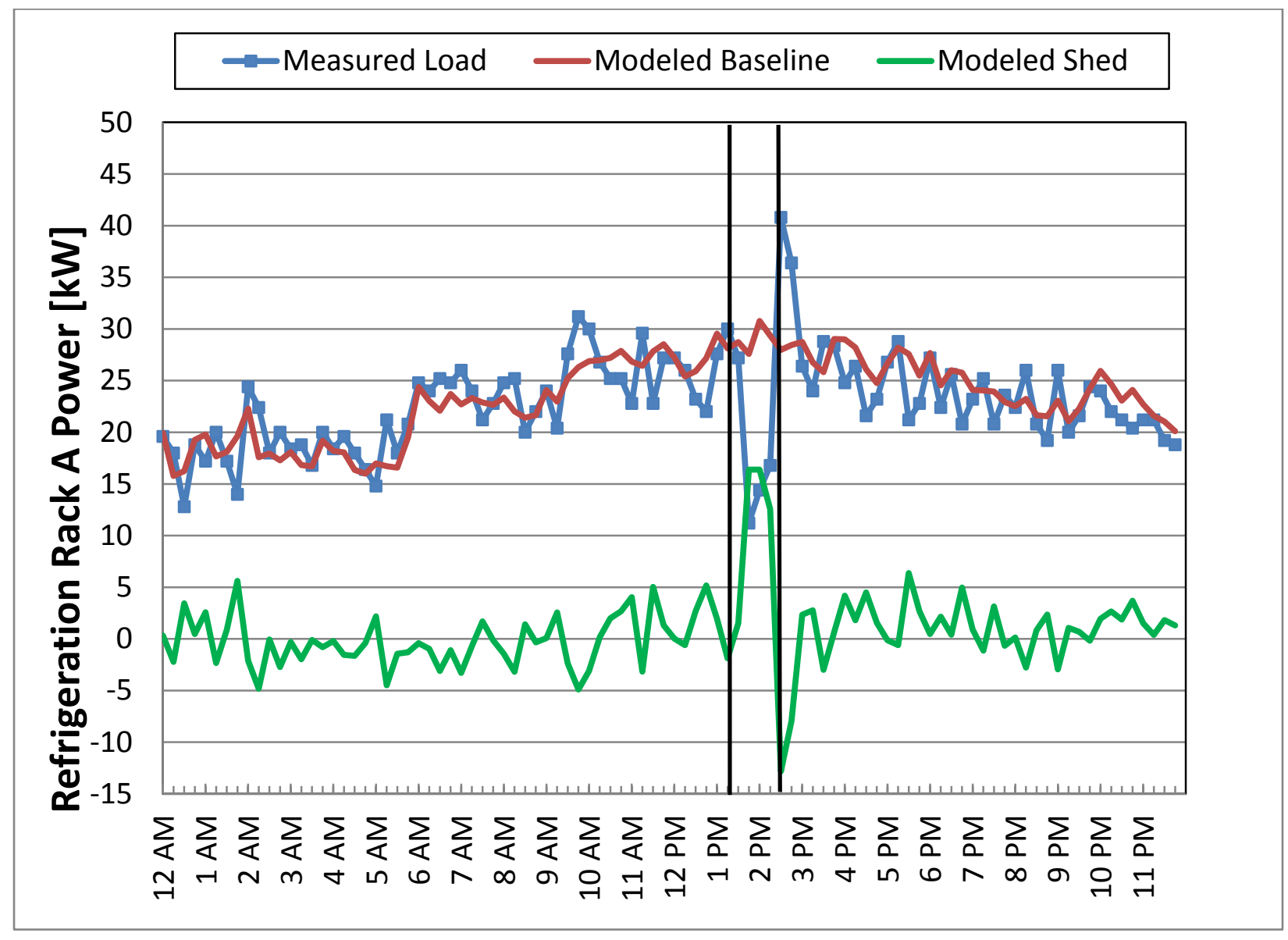

Figure 9. DR event at TAN WFM, Nov. 10, 2014

\subsubsection{Test \#3: Tanasbourne, July 14, 2015}

In this test, the team investigated how much load could be shed by setting the critical case set point at $-5^{\circ} \mathrm{F}$, still within the supermarket's recommendation, even after the product had reached this limit. In this test, the team expected the system to shut off completely for a short period of time before the product reached the threshold, and then operate at a slightly lower power draw for the remainder of the event, by maintaining the critical case at $-5^{\circ} \mathrm{F}$ rather than $-8^{\circ} \mathrm{F}$. The test was run for 4 hours (12-4 p.m.), leaving noncritical case and walk-in set points at their default 
values. The resulting change in power consumption and other pertinent test results are summarized in Table 7 and Figure 10.

Table 7. Overview of TAN WFM, July 14, 2015

\begin{tabular}{lc}
\hline Parameter & Value during DR Test \\
\hline Load shed range $\pm 95 \%(\mathrm{~kW})$ & $-1.3-8.3$ \\
\hline Average load shed $(\mathrm{kW})$ & 3.5 \\
\hline Outdoor air temperature range $\left({ }^{\circ} \mathrm{F}\right)$ & $87.4-92.3$ \\
\hline Average outdoor air temperature $\left({ }^{\circ} \mathrm{F}\right)$ & 90.2 \\
\hline Baseline load range $(\mathrm{kW})$ & $35.2-42.7$ \\
\hline Average baseline load $(\mathrm{kW})$ & 38.9 \\
\hline
\end{tabular}

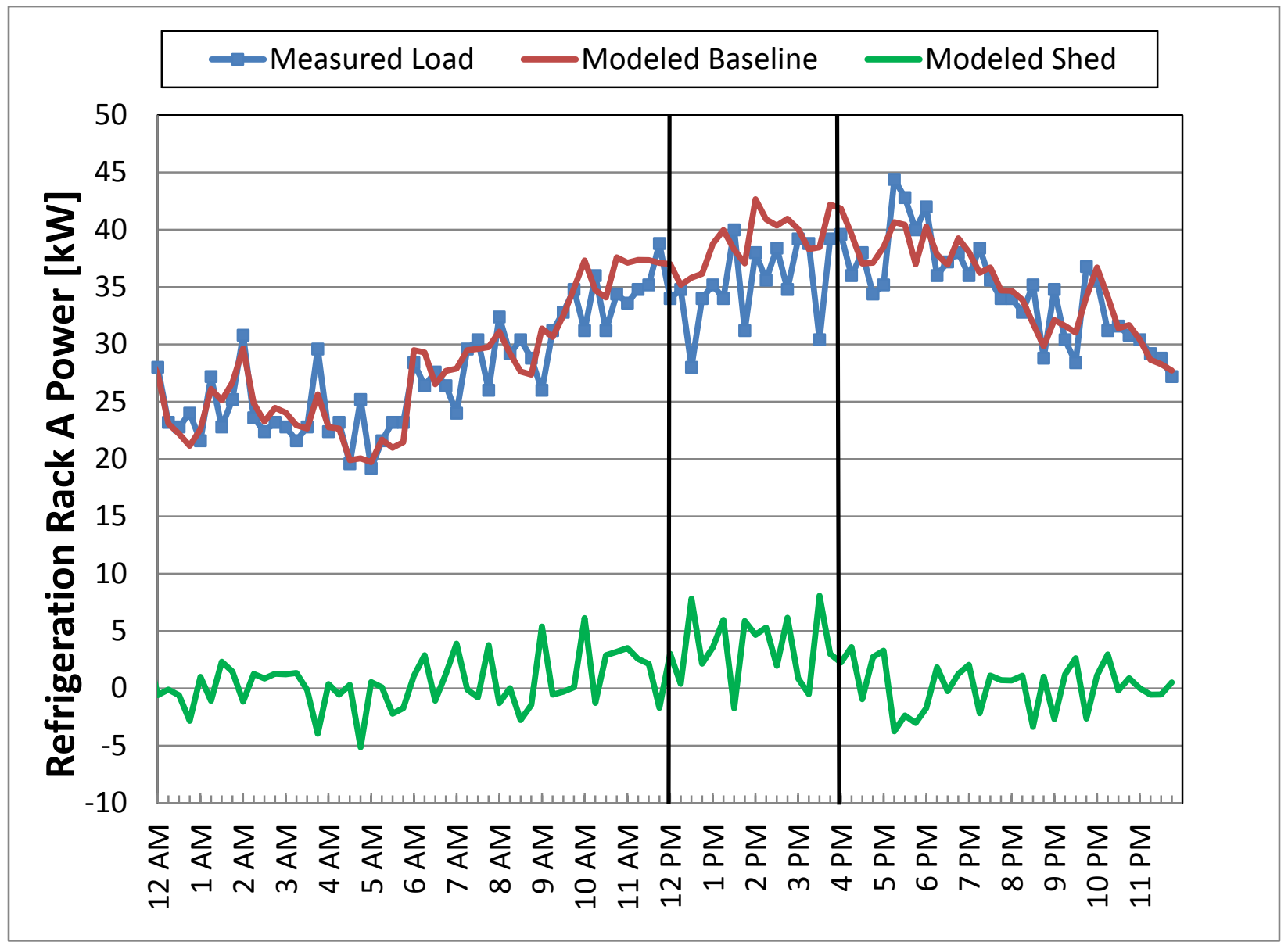

Figure 10. DR event at TAN WFM, July 14, 2015

\subsubsection{Test \#4: Tanasbourne, July 20, 2015}

In this test, the team attempted to add further power shed by increasing the critical and noncritical case set points. The team hypothesized that in addition to the benefits realized from 
increasing the saturated suction pressure of the rack, the team would see additional benefits from reducing the overall cooling load on the rack.

To this end, the team raised the float control for the rack from $-8^{\circ} \mathrm{F}$ to $-5^{\circ} \mathrm{F}$ for 4 hours $(12: 30-$ 4:30 p.m.), and changed noncritical case and walk-in set points to $+15^{\circ} \mathrm{F}$.

Table 8 and Figure 11 show that this modification increased the power shed significantly, even though the saturated suction pressure control scheme was consistent with Test \#3.

Table 8. Overview of TAN WFM, July 20, 2015

\begin{tabular}{lcc}
\hline Parameter & Value during DR Test \\
\hline Load shed range $\pm 95 \%(\mathrm{~kW})$ & $3.2-13.6$ \\
\hline Average load shed $(\mathrm{kW})$ & 8.4 \\
\hline Outdoor air temperature range $\left({ }^{\circ} \mathrm{F}\right)$ & $90.0-94.3$ \\
\hline Average outdoor air temperature $\left({ }^{\circ} \mathrm{F}\right)$ & 92.2 \\
\hline Baseline load range $(\mathrm{kW})$ & $35.5-44.3$ \\
\hline Average baseline load $(\mathrm{kW})$ & 40.6 \\
\hline
\end{tabular}




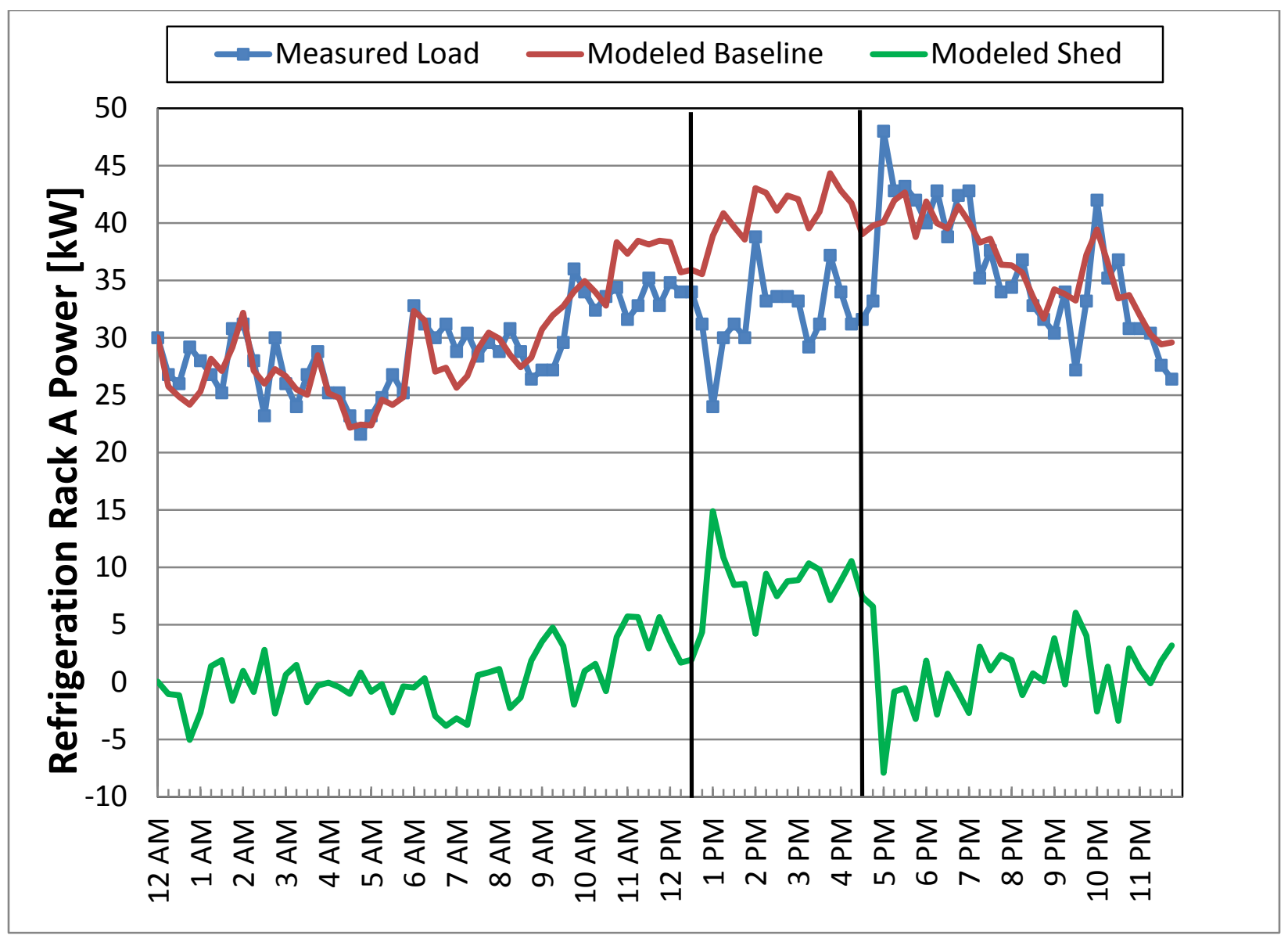

Figure 11. DR event at TAN WFM, July 20, 2015

\subsubsection{Test \#5: Tanasbourne, July 22, 2015}

In this test, the team investigated whether the addition of a precooling period before a DR event generated increased power shed. The team hypothesized that if the team were to lower the product temperatures below their normal temperatures before the event, that additional load shed would be possible. To this end, the team cooled the entire low-temperature system, including the critical and noncritical cases and walk-ins, to $-12^{\circ} \mathrm{F}$ from 10 a.m. to 1:15 p.m. Then from 1:155:15 p.m., the team raised the float control from $-8^{\circ} \mathrm{F}$ to $-5^{\circ} \mathrm{F}$ and the noncritical case DAT set point to $+15^{\circ} \mathrm{F}$. 
Table 9. Overview of TAN WFM, July 22, 2015

\begin{tabular}{lc}
\hline Parameter & Value during DR Test \\
\hline Load shed range $\pm 95 \%(\mathrm{~kW})$ & $-1.9-9.2$ \\
\hline Average load shed $(\mathrm{kW})$ & 3.6 \\
\hline Outdoor air temperature range $\left({ }^{\circ} \mathrm{F}\right)$ & $71.4-75.2$ \\
\hline Average outdoor air temperature $\left({ }^{\circ} \mathrm{F}\right)$ & 73.8 \\
\hline Baseline load range $(\mathrm{kW})$ & $29.1-35.9$ \\
\hline Average baseline load $(\mathrm{kW})$ & 32.6 \\
\hline Average additional precool power draw $(\mathrm{kW})$ & 7.7 \\
\hline
\end{tabular}

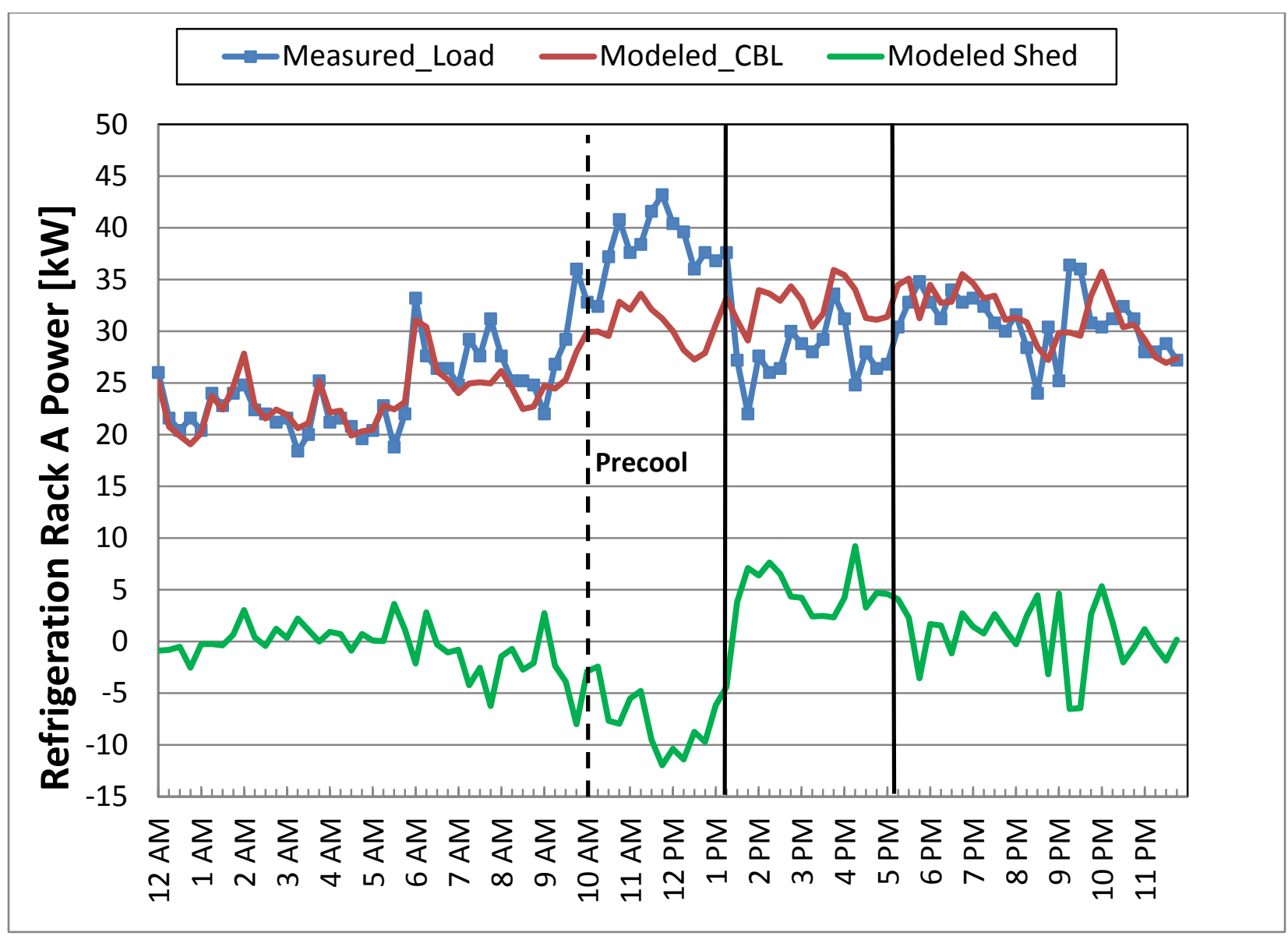

Figure 12. DR event at TAN WFM, July 22, 2015

\subsubsection{Test \#6: Tanasbourne, July 24, 2015}

In this test, the team investigated whether the benefits of precooling would allow significant load shed to occur while only modifying the critical case set point, and with it the SST. To this end, the team cooled the entire low-temperature system including critical and non 
critical cases and walk-ins to $-12^{\circ} \mathrm{F}$ from 10:30 a.m. to 1:30 p.m., then changed the set point of the critical case to $-5^{\circ} \mathrm{F}$ for 4 hours, leaving the noncritical case and walk-in set points at their default values.

The resulting change in power consumption and other pertinent test results are summarized in Table 10 and Figure 13. This test confirmed suspicions that simply modifying the saturated suction pressure of the rack does not result in substantial load shedding capacity, even with precooling, at least on a relatively hot day.

Table 10. Overview of TAN WFM, July 24, 2015

\begin{tabular}{lc}
\hline Parameter & Value during DR Test \\
\hline Load shed range $\pm 95 \%(\mathrm{~kW})$ & $-3.3-7.6$ \\
\hline Average load shed $(\mathrm{kW})$ & 2.1 \\
\hline Outdoor air temperature range $\left({ }^{\circ} \mathrm{F}\right)$ & $84.9-91.4$ \\
\hline Average outdoor air temperature $\left({ }^{\circ} \mathrm{F}\right)$ & 88 \\
\hline Baseline load range $(\mathrm{kW})$ & $35.4-42.0$ \\
\hline Average baseline load $(\mathrm{kW})$ & 39.2 \\
\hline Average additional precool power draw $(\mathrm{kW})$ & 3.5 \\
\hline
\end{tabular}




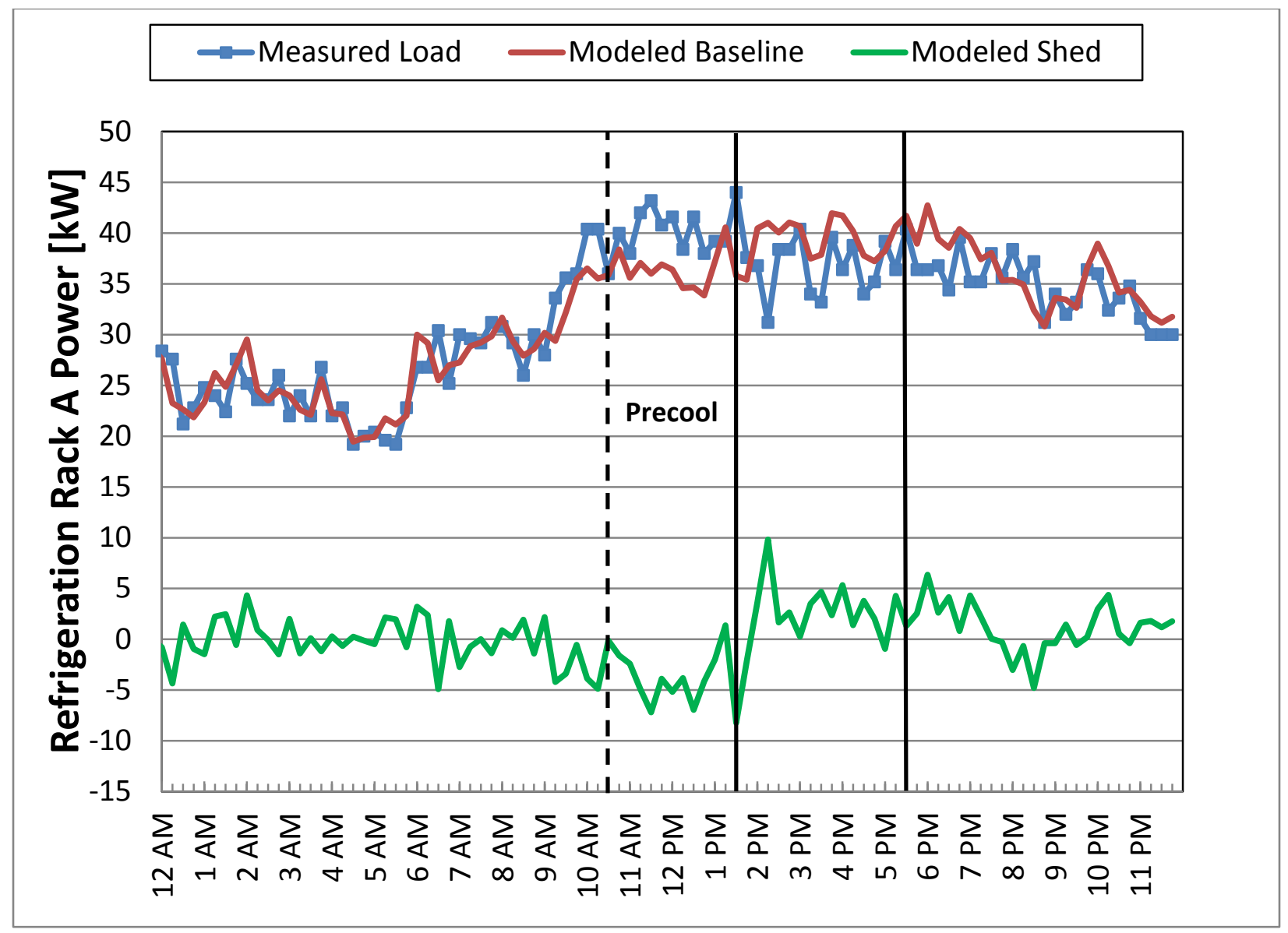

Figure 13. DR event at TAN WFM, July 24, 2015

\subsubsection{Test \#7: Tanasbourne, July 28, 2015}

In this test, the team attempted to isolate the effect of load reduction by raising the set points of the noncritical cases and walk-ins only to $+15^{\circ} \mathrm{F}$. The resulting change in power consumption and other pertinent test results are summarized in Table 11 and Figure 14. The results suggest that in order to achieve significant load shedding, both the SST and the rack's cooling load must be changed.

Table 11. Overview of TAN WFM, July 28, 2015

\begin{tabular}{lc}
\hline Parameter & Value during DR Test \\
\hline Load shed range $\pm 95 \%(\mathrm{~kW})$ & $-3.1-6.8$ \\
\hline Average load shed $(\mathrm{kW})$ & 1.9 \\
\hline Outdoor air temperature range $\left({ }^{\circ} \mathrm{F}\right)$ & $87.1-92.1$ \\
\hline Average outdoor air temperature $\left({ }^{\circ} \mathrm{F}\right)$ & 89.3 \\
\hline Baseline load range $(\mathrm{kW})$ & $35.5-41.8$ \\
\hline Average baseline load $(\mathrm{kW})$ & 38.3 \\
\hline
\end{tabular}




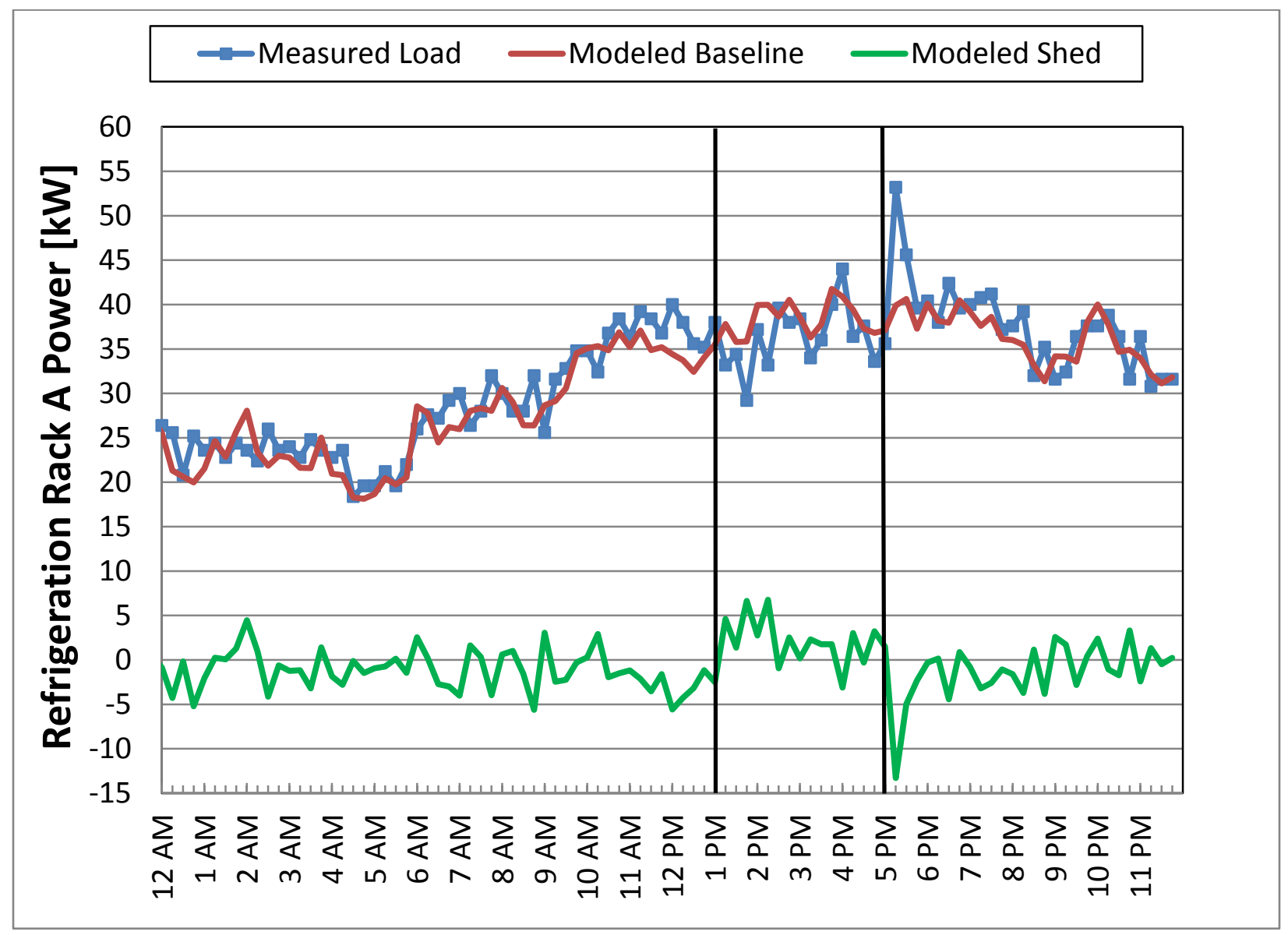

Figure 14. Demand response event at Tanasbourne Whole Foods Market, July 28, 2015

\subsubsection{Test \#8: Tanasbourne, July 30, 2015}

In this test, the team attempted to ascertain an upper estimate of available DR by using best practices learned from previous tests. To this end, the team employed every strategy that had proven beneficial on one of the hotter days of the year. The team cooled the entire lowtemperature system including critical and noncritical cases and walk-ins to $-12^{\circ} \mathrm{F}$ from $11 \mathrm{a} . \mathrm{m}$. to 2 p.m. The team then changed the float control of the critical case (and thus the rack) from $-8^{\circ} \mathrm{F}$ to $-5^{\circ} \mathrm{F}$, and changed the set points of the noncritical cases to $+15^{\circ} \mathrm{F}$ from $0^{\circ} \mathrm{F}$. The test was done for what the team assumed would be the 6 hottest hours of the day, from $2-8$ p.m.

The resulting change in power consumption and other pertinent test results are summarized in Table 11 and Figure 14. They show a relatively large benefit from the precooling at the beginning of the event, with load shedding maintaining values above $10 \mathrm{~kW}$ for most of the first three hours of the test. However, around 5 p.m., the amount of load shed dropped, possibly due to increased customer traffic after the workday. 
Table 12. Overview of TAN WFM, July 30, 2015

\begin{tabular}{lc}
\hline Parameter & Value during DR Test \\
\hline Load shed range $\pm 95 \%(\mathrm{~kW})$ & $2.5-11.9$ \\
\hline Average load shed $(\mathrm{kW})$ & 7.2 \\
\hline Outdoor air temperature range $\left({ }^{\circ} \mathrm{F}\right)$ & $95.5-111.9$ \\
\hline Average outdoor air temperature $\left({ }^{\circ} \mathrm{F}\right)$ & 105.8 \\
\hline Baseline load range $(\mathrm{kW})$ & $43.3-50.7$ \\
\hline Average baseline load $(\mathrm{kW})$ & 47.2 \\
\hline Average additional precool power draw $(\mathrm{kW})$ & 4.6 \\
\hline
\end{tabular}

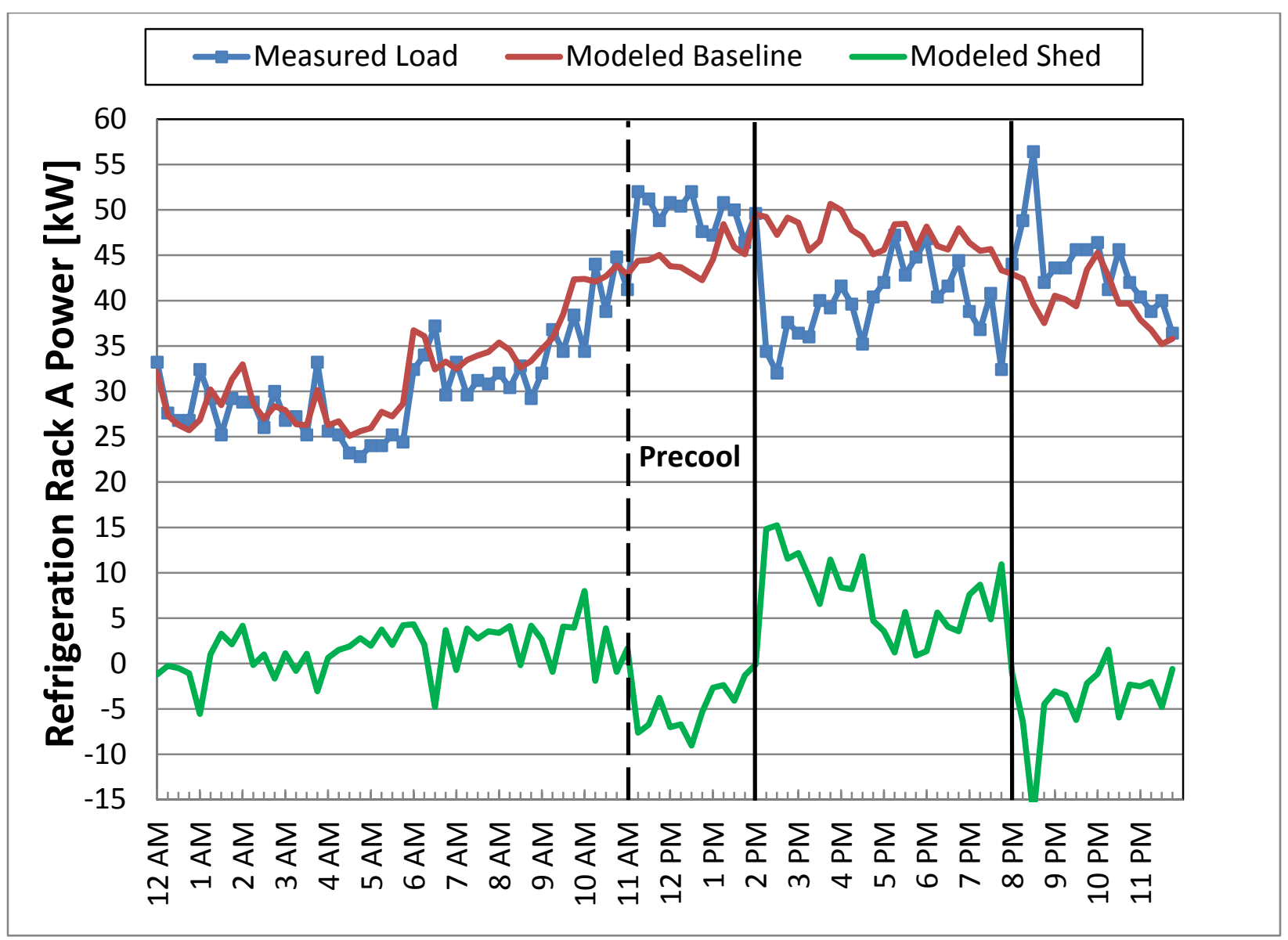

Figure 15. DR event at TAN WFM, July 30, 2015

\subsubsection{Test \#9: Tanasbourne, July 31, 2015}

In this test, the team attempted to isolate the effects of precooling by cooling the entire lowtemperature system to $-12^{\circ} \mathrm{F}$ for 3 hours (11:30 a.m. to 2:30 p.m.) and then returning the system to its default operation. The resulting change in power consumption and other pertinent test results are summarized in Table 13 and Figure 16. This test showed that precooling alone is not a viable DR strategy, as the test actually increased the average load. 
Table 13. Overview of TAN WFM, July 31, 2015

\begin{tabular}{lc}
\hline Parameter & Value during DR Test \\
\hline Load shed range $\pm 95 \%(\mathrm{~kW})$ & $-7.3-3.1$ \\
\hline Average load shed $(\mathrm{kW})$ & -2.1 \\
\hline Outdoor air temperature range $\left({ }^{\circ} \mathrm{F}\right)$ & $104.4-108.5$ \\
\hline Average outdoor air temperature $\left({ }^{\circ} \mathrm{F}\right)$ & 106.1 \\
\hline Baseline load range $(\mathrm{kW})$ & $44.4-49.4$ \\
\hline Average baseline load $(\mathrm{kW})$ & 46.7 \\
\hline Average additional precool power draw $(\mathrm{kW})$ & 5.7 \\
\hline
\end{tabular}

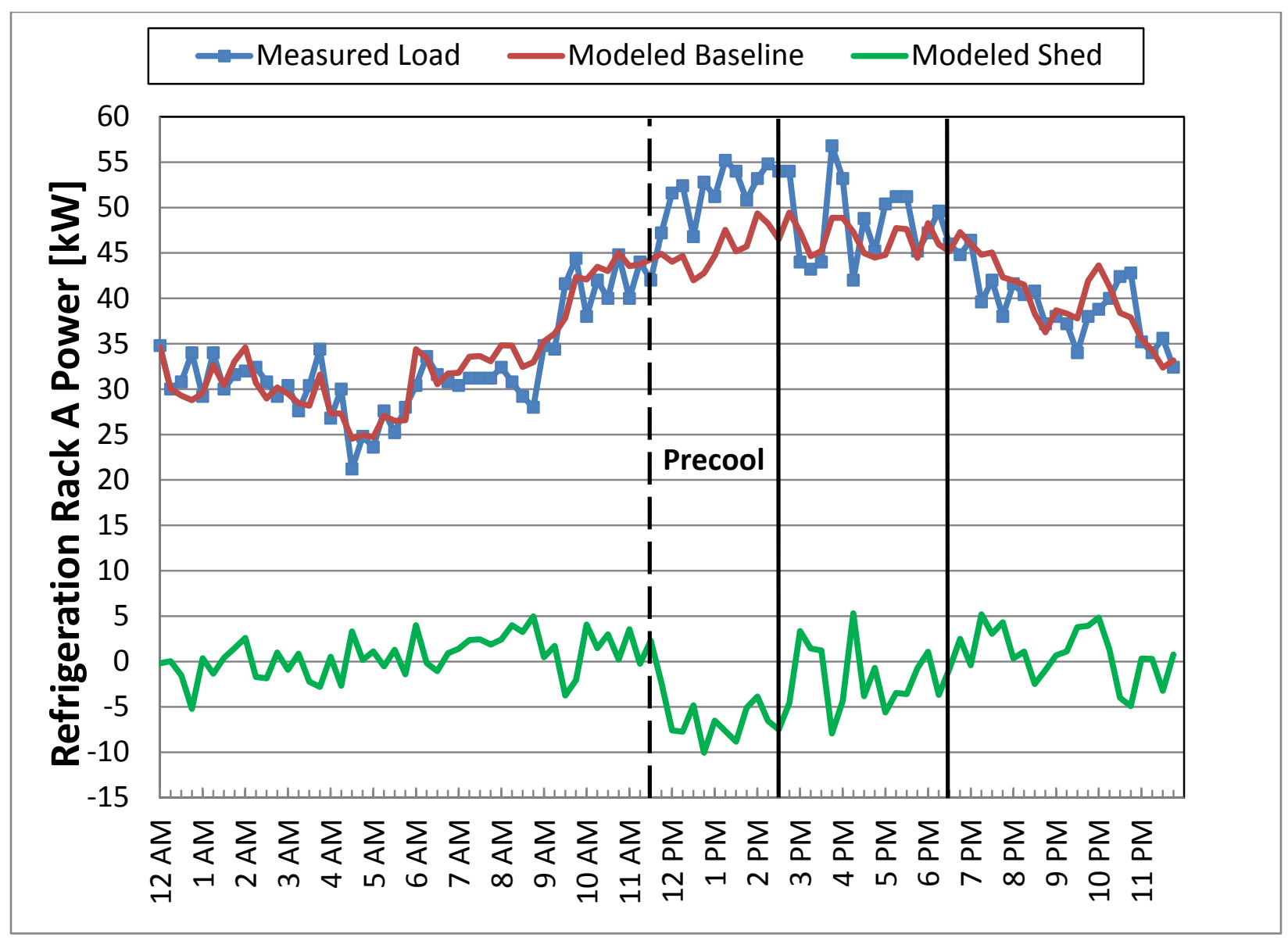

Figure 16. DR event at TAN WFM, July 31, 2015

\subsection{Model Identification Experiments}

Separate sets of tests were conducted to generate data for gray-box model training and validation. Data collected during the training (Figure 18 and Figure 20) and validation (Figure 21 and Figure 22) tests are shown below for the vertical and walk-in cases. These figures are included to show that the cases were excited thermally throughout the tests, which was a 
requirement for developing accurate models, and to show the reader the vast difference in the respective rates of response of the case air and the product simulator.

\subsubsection{Tanasbourne Pseudo-Random Binary Signal Training, July 21, 2015}

Temperature set point signals to circuits A2 (Walk-In Seafood) and A8 (Reach-In Frozen Food) were varied between $15^{\circ} \mathrm{F}$ and $0^{\circ} \mathrm{F}$ with a PRBS as explained above. This activated the EEPRs on each circuit via the existing control system. Resulting product and air temperatures were recorded for model development.

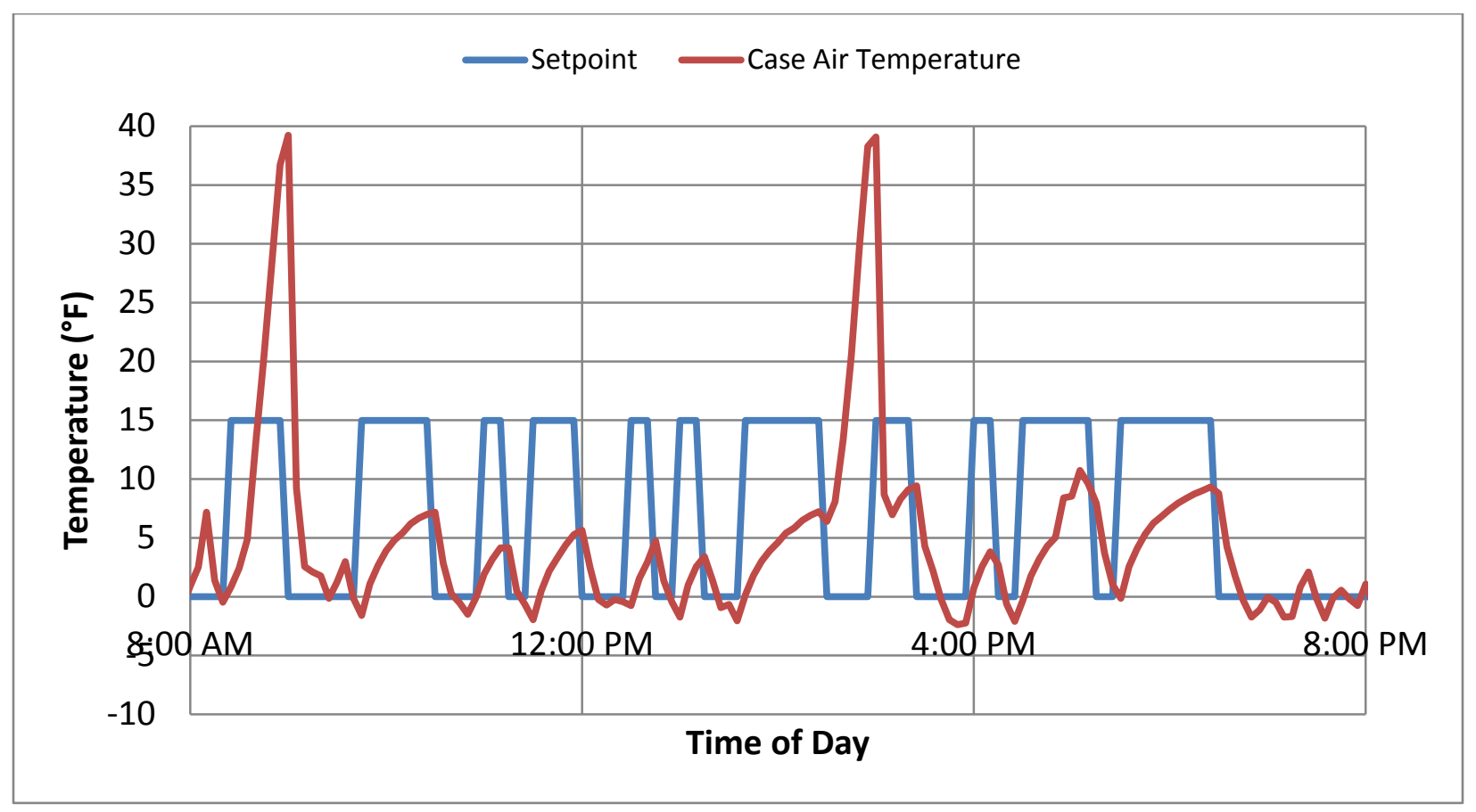

Figure 17. Walk-in air temperature and set point during training test 


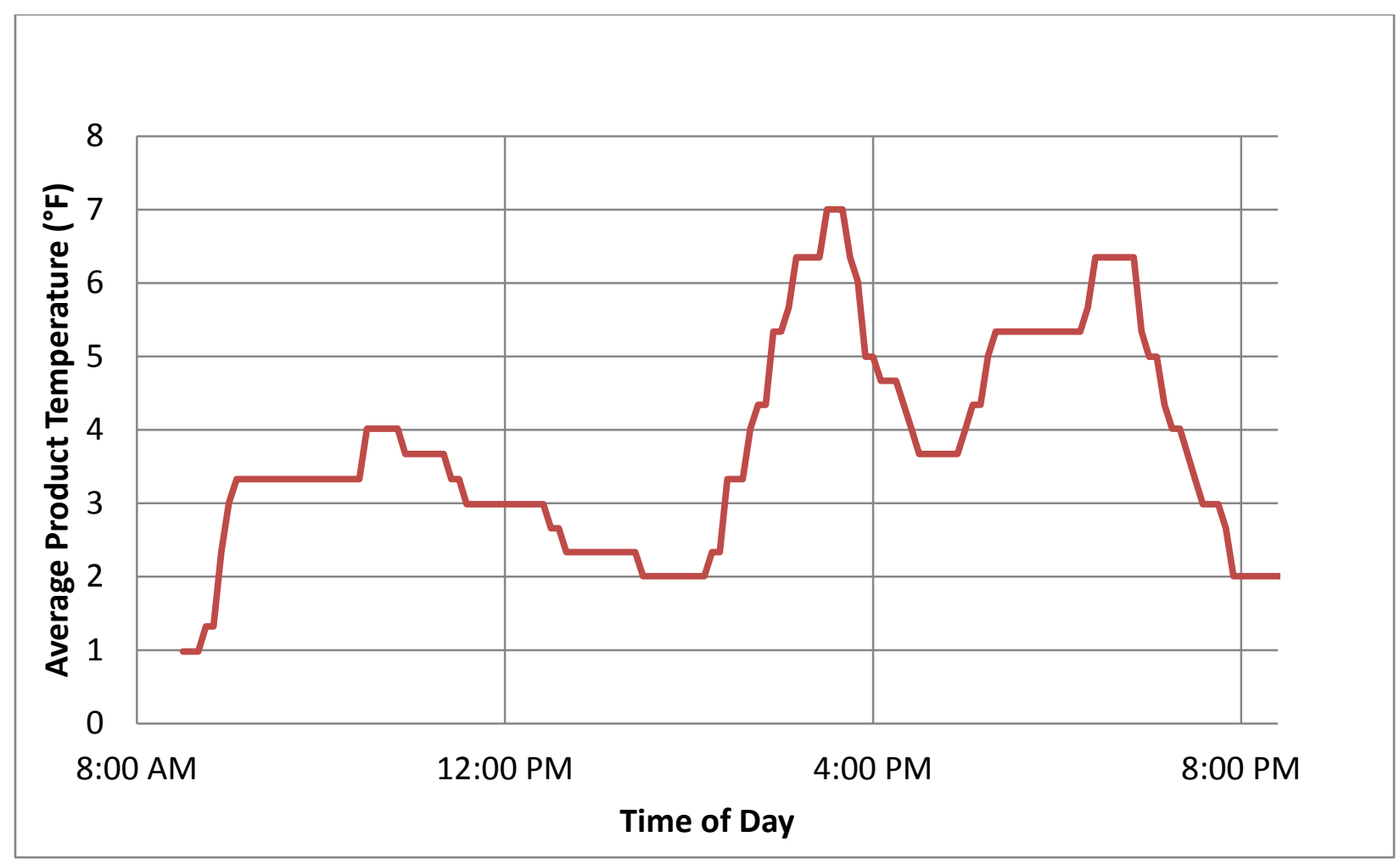

Figure 18. Walk-in product temperature during training test

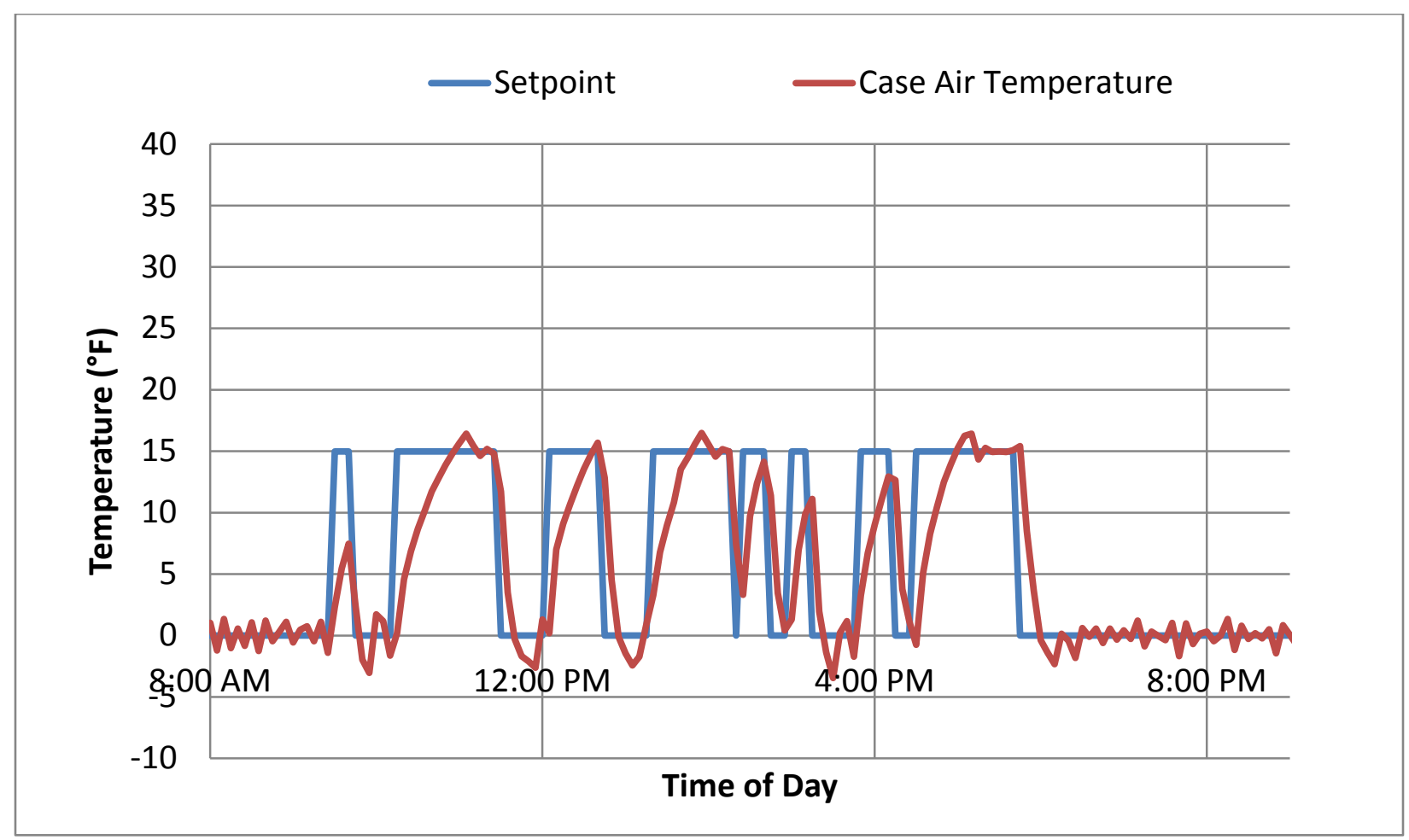

Figure 19. Reach-in air temperature and set point during training test 


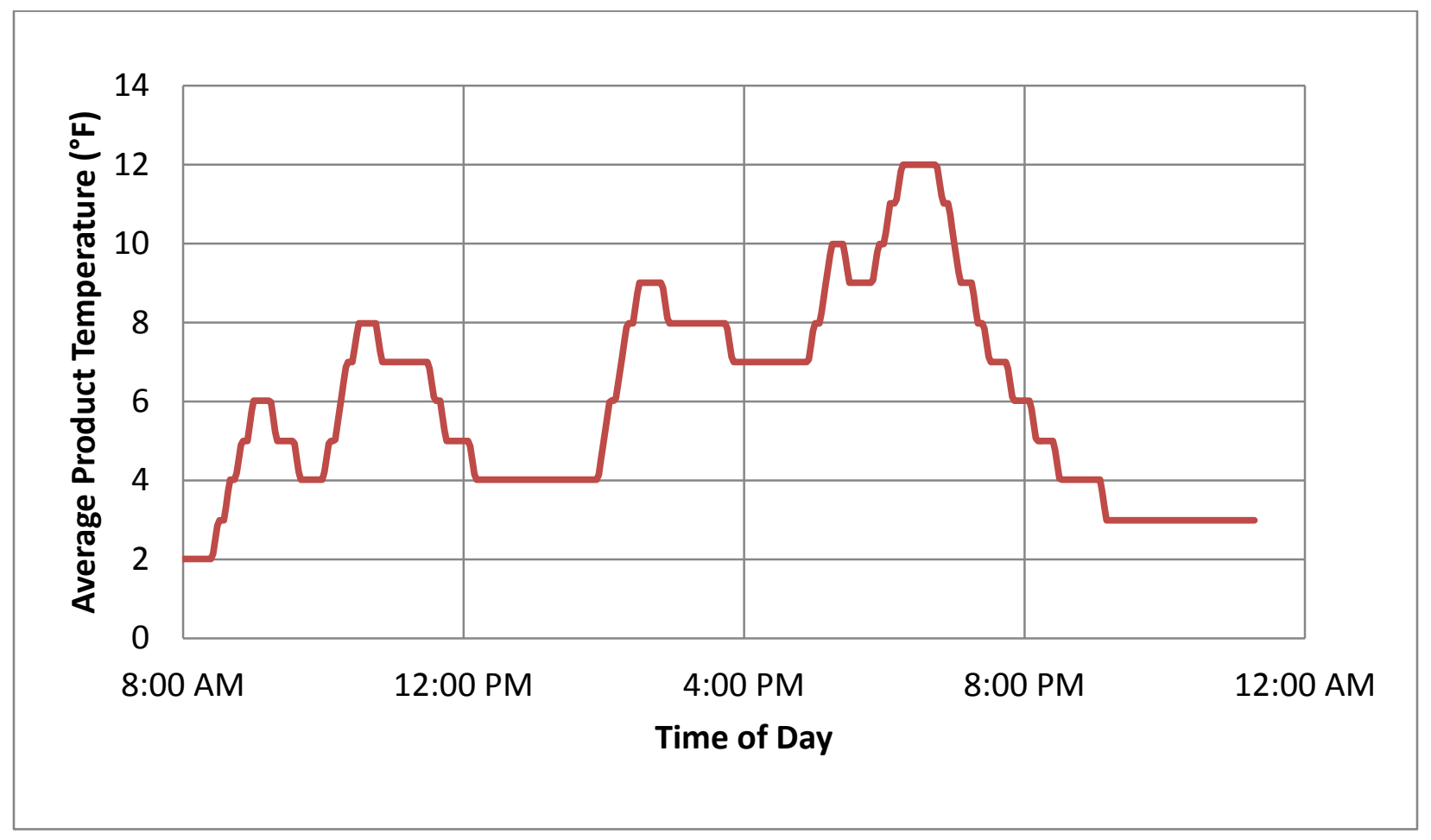

Figure 20. Reach-in product temperature during training test

\subsubsection{Tanasbourne Pseudo-Random Binary Signal Validation, July 23, 2015} An additional set of tests was conducted on July 23, 2015. The data generated in these tests was used to validate the model generated from the July 21, 2015 data. The system was excited in a fashion similar to that of the training set. Resulting product temperatures are shown below. 


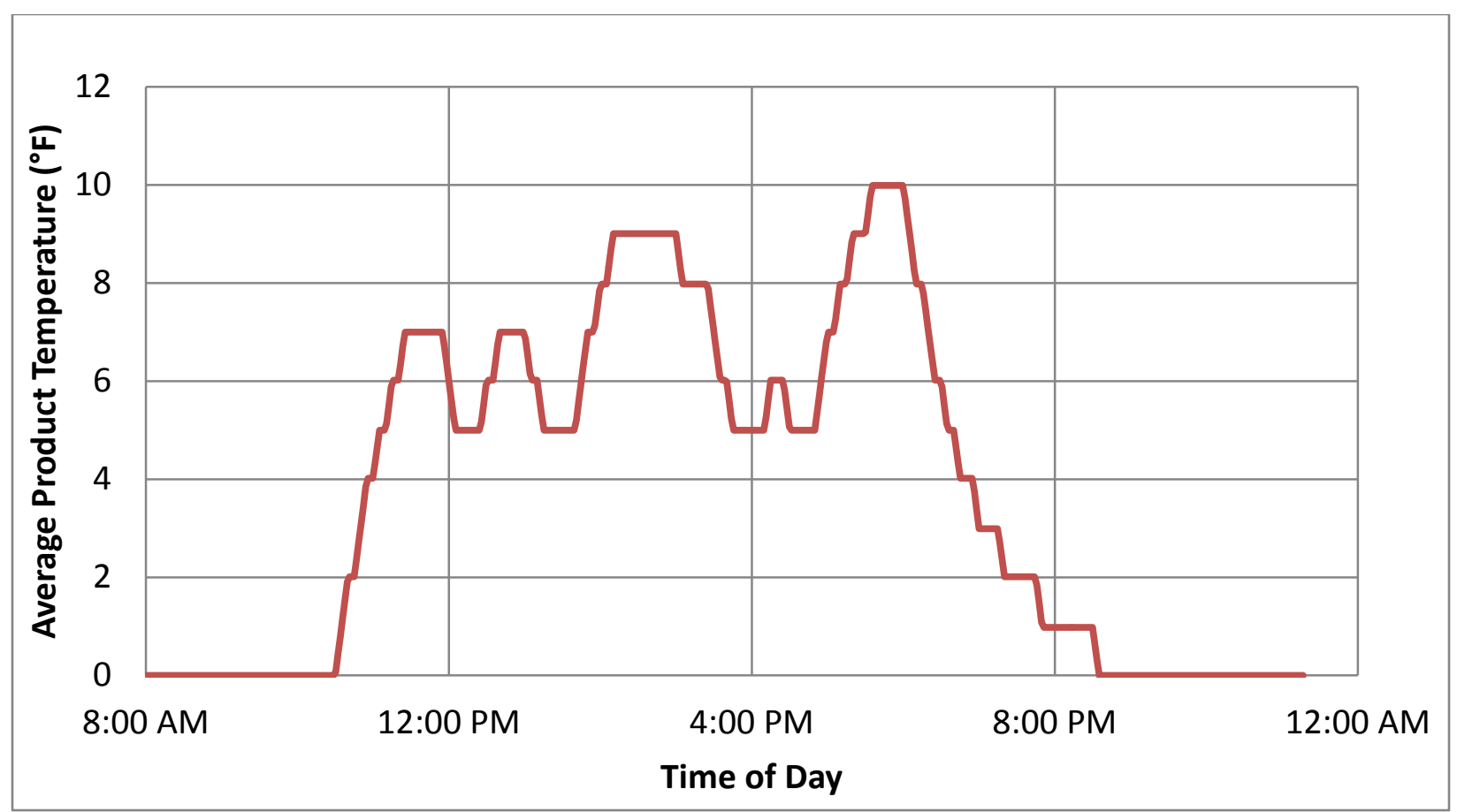

Figure 21. Reach-in product temperature during validation test

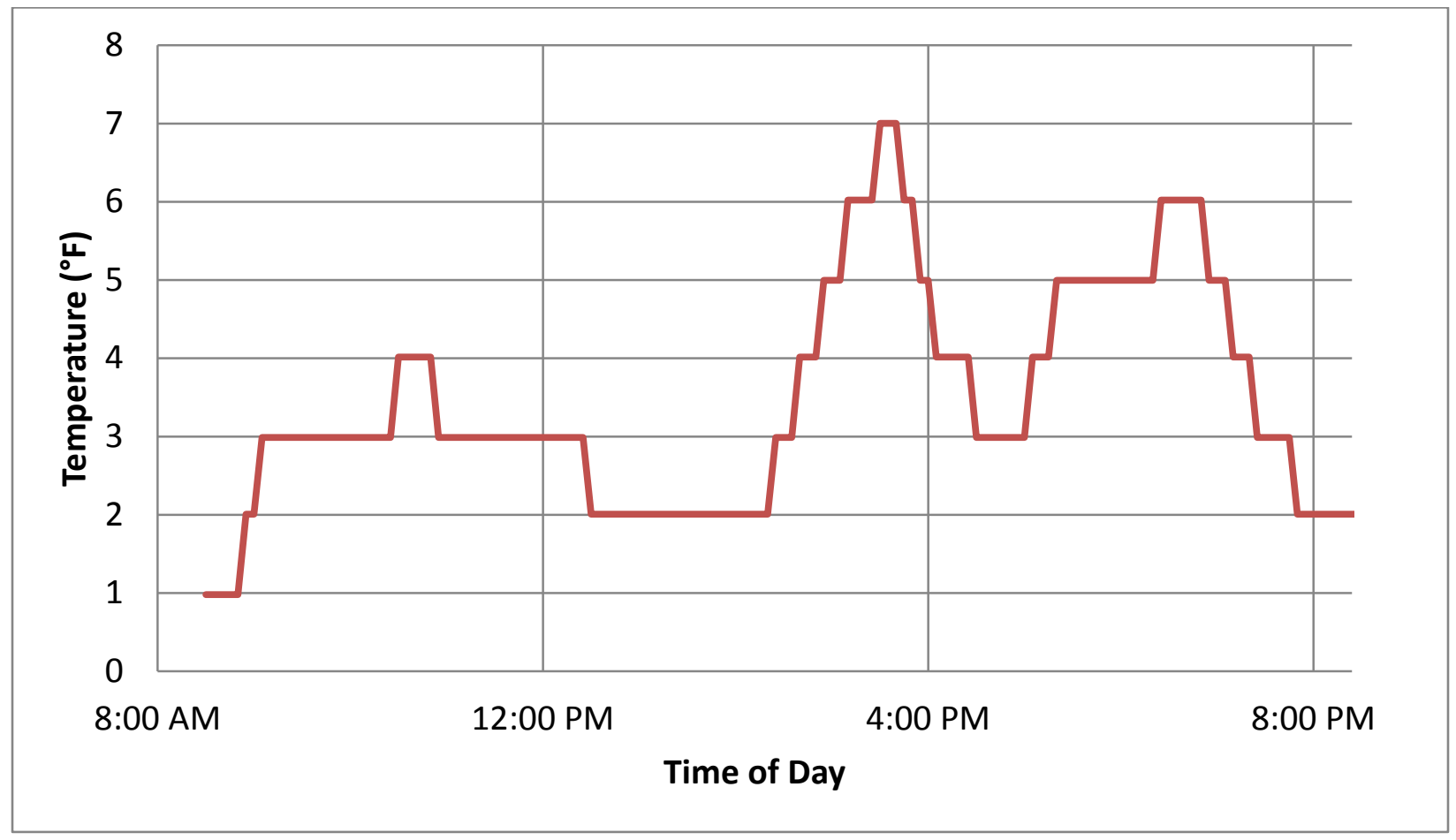

Figure 22. Walk-in product temperature during validation test 


\section{Simulation Results}

Simulations were first conducted to validate the new models the team developed. Then a second set of simulations was done to quantify DR resources available in BPA's service territory. Results are presented in this section.

\subsection{Model Validation}

Two model validations were conducted. The first compared the performance of the thermal model, which was concerned exclusively with the dynamics of the temperatures within the refrigerated display cases. The second looked at the model's ability to predict power consumption during a DR test by comparing the model output to data from the actual store.

\subsubsection{Thermal Model}

Models were developed using the gray-box approach with the training test data. Multiple model structures were tested for their ability to predict the validation data accurately and provide the information needed for DR calculations. Figure 23 and Figure 24 show the data collected from TAN. Its measurement uncertainty is shown overlaid with the data generated by the models developed in this project.

As can be seen, the model predicts the dynamics in the case extremely accurately. The reach-in dataset is predicted with a root mean squared error (RMSE) of $0.40^{\circ} \mathrm{F}$ and the walk-in, $0.57^{\circ} \mathrm{F}$. Each of these models takes only the control signals (SST and case set point) as inputs, as is the case for manipulation of a real store's refrigeration system.

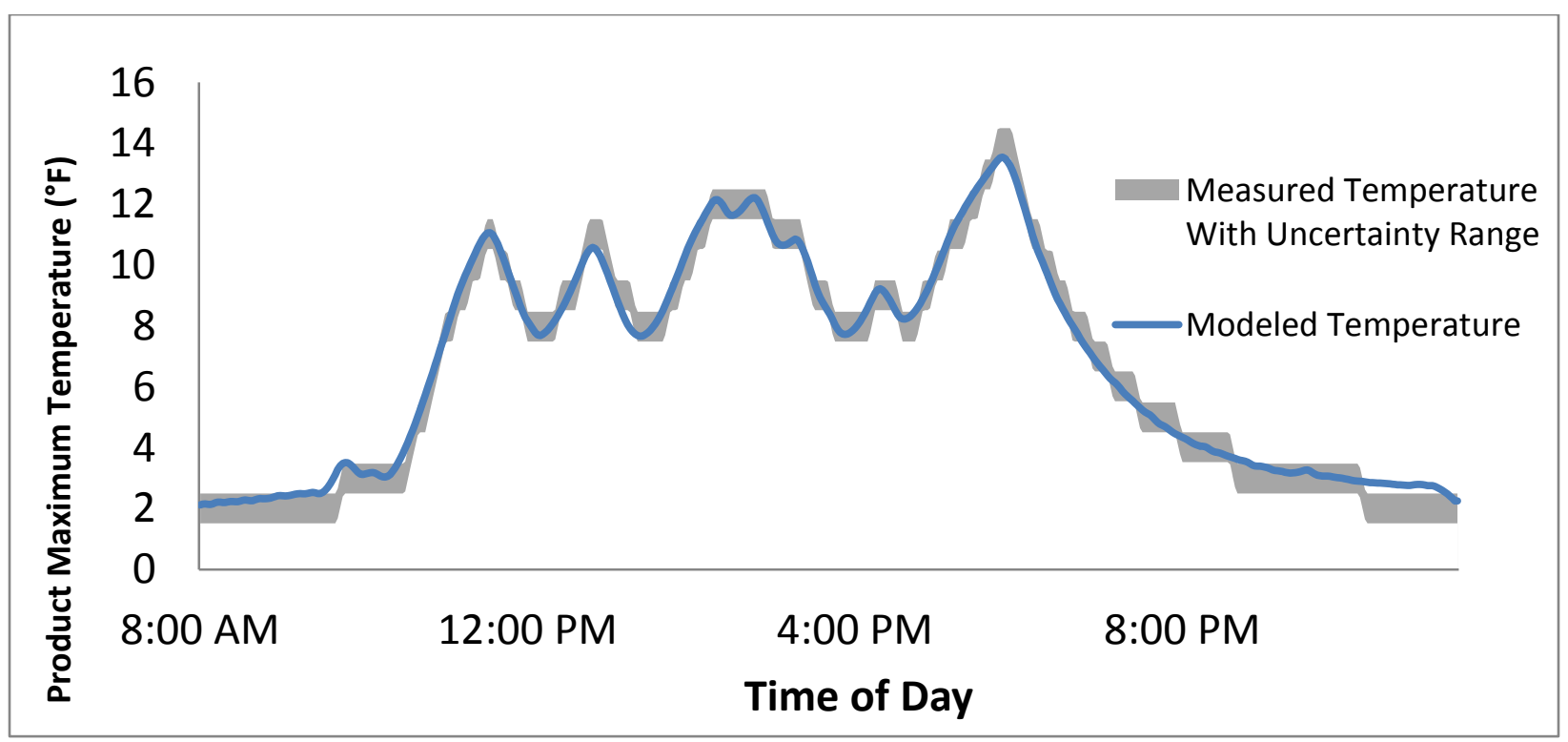

Figure 23. Reach-in validation: maximum temperature comparison $(\mathrm{RMSE}=\mathbf{0 . 4 0 1 )}$ 


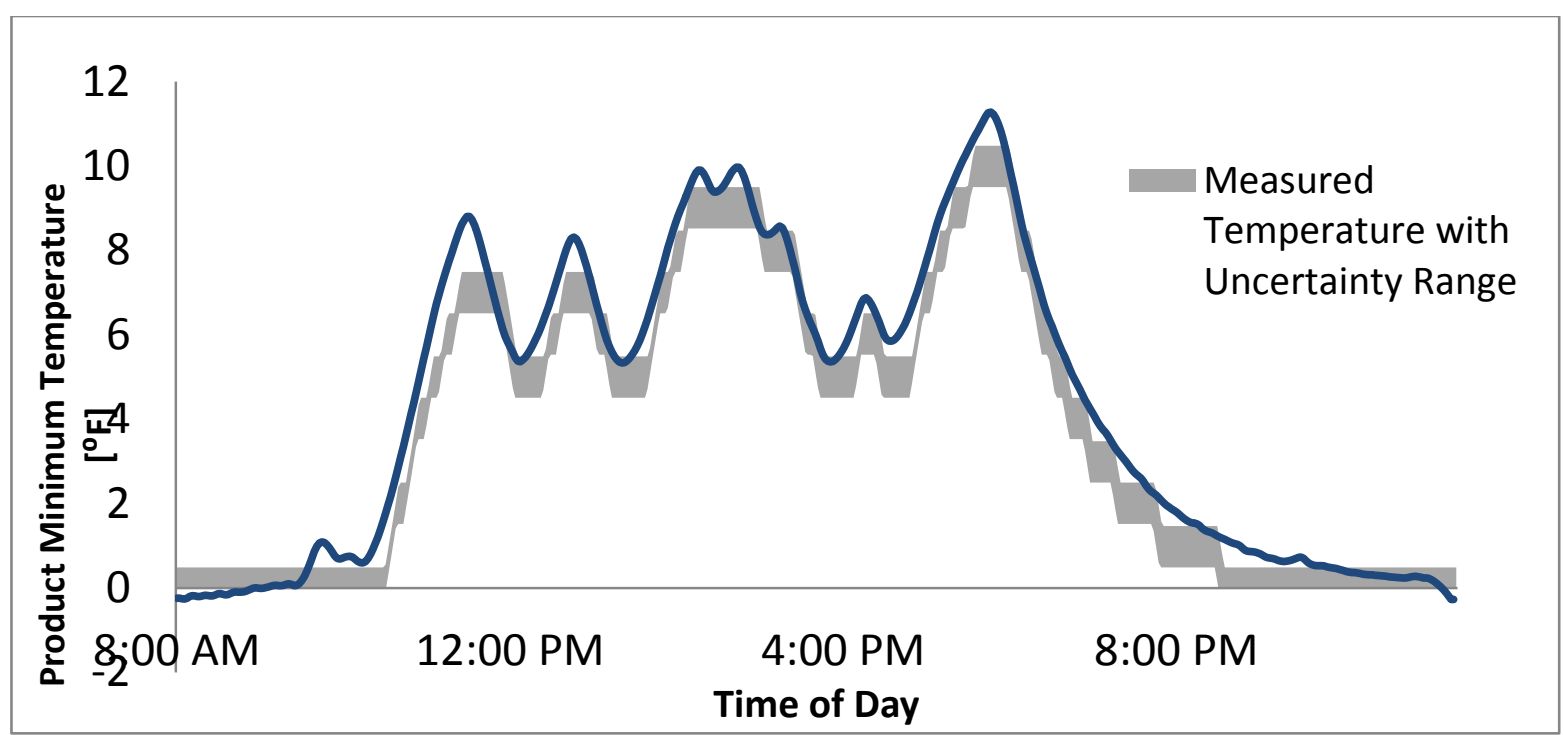

Figure 24. Walk-in validation: maximum temperature comparison $(\mathrm{RMSE}=\mathbf{0 . 5 6 6})$

\subsubsection{Model of Electrical Power Use}

With the thermal model developed and matched very closely to measured data, the team compared the electrical power shed available in the low-temperature cases with that measured during an actual test on July 20, 2015, at TAN. This task was rather difficult, as the site submetering of electrical power was performed at the rack level and the rack that was used for testing included a few medium-temperature cases. WFM did not allow us to change operation of medium-temperature cases out of concern for product quality, and thus our models do not include the effect on any medium-temperature cases. Therefore, the shed that is labeled "measured" in Figure 25 is a value for normal operation, calculated according to the procedure described in Section 4.4, minus the measured power during the DR test. The modeled power is generated knowing only outdoor conditions and set points.

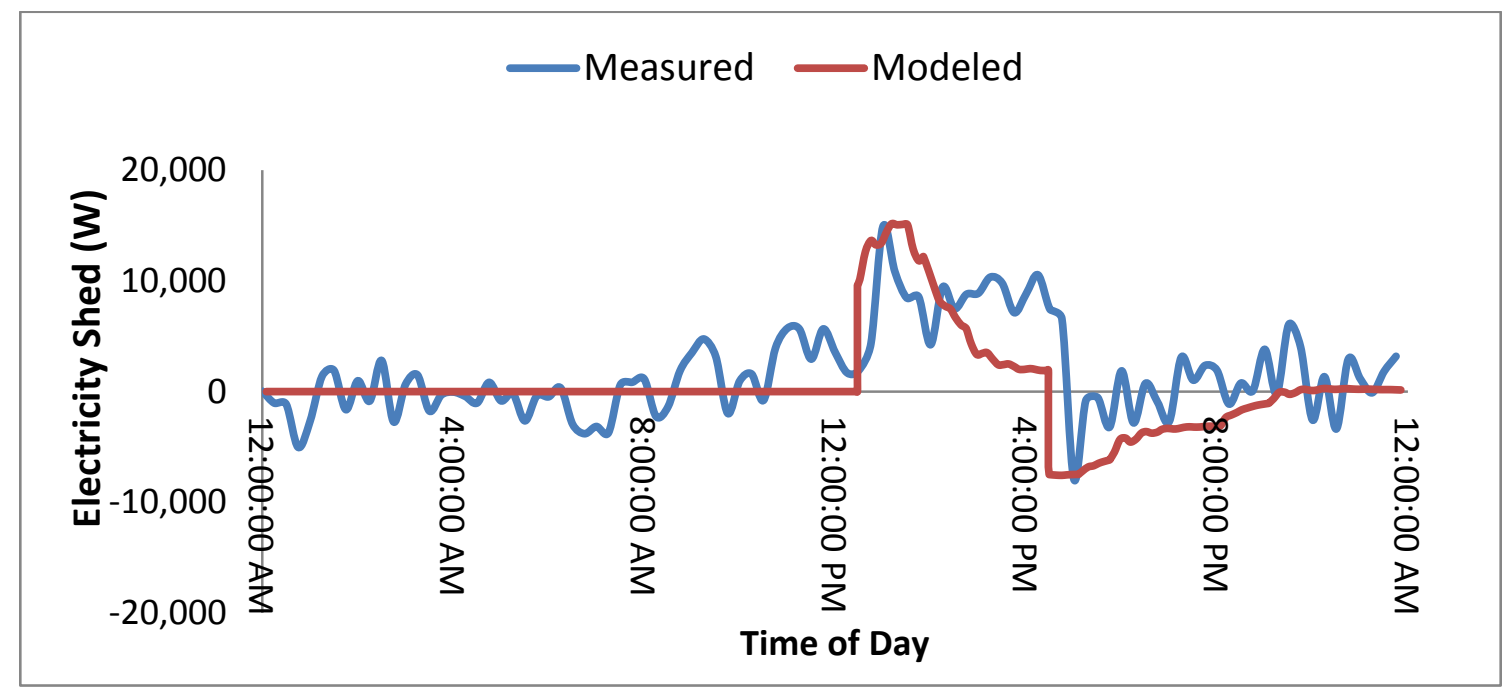

Figure 25. Comparison between modeled and "measured" electrical power during demand response 
As can be seen in Figure 25, the model captures both the peak electricity shed and the peak rebound power draw well, and can be considered conservative in regards to how long the power shed is available and the power required during rebound after the DR event. The models assume a continuously variable capacity system, which was not present in the actual store, and have other inherent assumptions that smooth the output in comparison to the actual store power. Nonetheless, these models give very reliable estimates of the power savings available in supermarkets.

\subsection{Demand Response Simulations}

Using the calibrated and validated models, the team conducted analyses to obtain information not available from the field tests. This information includes how much DR is available in different locations within BPA's service territory, how DR events can be expected to look on critical days of the year, and theoretical limits on potential when equipment suitable for this type of operation is included in the store (e.g., variable capacity compressors, properly organized suction groups).

The team assumed for all simulations that the amount of refrigeration capacity of each type of case was equal to that of the U.S. Department of Energy supermarket reference building (Doebber et al. 2015), and that all low-temperature cases (excluding ice cream) could be manipulated during a DR event. This was equivalent to changing approximately twice the capacity of cases as was done in the actual store.

To this end, four sets of simulations were conducted. Simulations were conducted for major population centers representing geographic and climatic variation within BPA's service area: Seattle, Washington; Portland, Oregon; Spokane, Washington; and Boise, Idaho. For DR simulations, the hottest day of 2014 was selected for each location (this varied by site) and compiled into a weather file used for the simulations. The team also ran a load-add simulation on April 21, 2014, for each location, to quantify the amount of additional demand that could be added.

The following simulations were performed for each location:

- In response to a ten-minute notice, the following changes were simulated:

- Set points for all critical (ice-cream) cases and walk-ins were raised from $-8^{\circ} \mathrm{F}$ to $-5^{\circ} \mathrm{F}$

○ The team allowed the SST to float upward $3^{\circ} \mathrm{F}$ in response to the change in the critical case

- Set points for all noncritical low-temperature cases and walk-ins (frozen food other than ice cream) were raised from $0^{\circ} \mathrm{F}$ to $+15^{\circ} \mathrm{F}$

- Set point changes were maintained for 90 minutes.

- In response to a day-before notice, the following changes were simulated:

- A 4-hour precool was scheduled in which all low-temperature case and walk-in set points were lowered to $-12^{\circ} \mathrm{F}$

- Set point modifications, identical to those listed for the 10-minute notice above, were specified, but held for a period of 4 hours. 
- To simulate added load during periods of excess supply, set points for all lowtemperature cases and walk-ins were lowered to $-12^{\circ} \mathrm{F}$ from $3-6$ a.m.

The charts in the Appendix show electric power time-series results for the simulations. Table 14 provides modeled results for the available load shed DR resource, the time that resource is available, and rebound demand at each location. Table 14 also provides details related to the load-add resource for the precooling simulations.

Table 14. Site-Specific Estimates of Total Refrigeration DR Resource, Rebound, and Load-Add Potential

\begin{tabular}{ll|cc|cc|cc}
\hline & & \multicolumn{2}{c}{ Precool } & \multicolumn{2}{c}{ DR } & \multicolumn{2}{c}{ Rebound } \\
\hline Location & Simulation & $\begin{array}{c}\text { Additional } \\
\text { Load (kW) }\end{array}$ & $\begin{array}{c}\text { Time } \\
\text { (h) }\end{array}$ & $\begin{array}{c}\text { Load Shed } \\
\text { (kW) }\end{array}$ & $\begin{array}{c}\text { Time } \\
\text { (h) }\end{array}$ & $\begin{array}{c}\text { Additional } \\
\text { Load (kW) }\end{array}$ & $\begin{array}{c}\text { Time } \\
\text { (h) }\end{array}$ \\
\hline Seattle & 10-Minute Notification & NA & NA & 18.5 & 1.25 & 6.5 & $3 \mathrm{~h}$ \\
\hline Spokane & 10-Minute Notification & NA & NA & 19.5 & 1.5 & 6.8 & 2.75 \\
\hline Portland & 10-Minute Notification & NA & NA & 15.0 & 1.5 & 5.3 & 2.75 \\
\hline Boise & 10-Minute Notification & NA & NA & 19.8 & 1.5 & 6.8 & 3 \\
\hline Seattle & Day-Before Notification & 6.8 & 4 & 17.8 & 2.5 & 6.6 & 3.5 \\
\hline Spokane & Day-Before Notification & 6.9 & 4 & 19.3 & 2.5 & 6.9 & 3.5 \\
\hline Portland & Day-Before Notification & 5.5 & 4 & 15.0 & 3 & 5.5 & 4 \\
\hline Boise & Day-Before Notification & 6.9 & 4 & 19.3 & 2.25 & 6.9 & 3.5 \\
\hline Seattle & Load-Add & 5.4 & 3 & N/A & N/A & -12.0 & 0.75 \\
\hline Spokane & Load-Add & 5.2 & 3 & N/A & N/A & -11.2 & 0.75 \\
\hline Portland & Load-Add & 5.5 & 3 & N/A & N/A & -12.0 & 0.75 \\
\hline Boise & Load-Add & 5.2 & 3 & N/A & N/A & -11.5 & 0.75 \\
\hline
\end{tabular}

Using data detailing the number and location (by region) of retail grocery establishments in the Pacific Northwest (Shelby Publishing Co., Inc. 2015), the team extrapolated the results from the calibrated model analysis to estimate the total DR resource available within BPA's service territory. Results of this analysis are shown in Table 15.

Table 15. Extrapolated BPA DR and Load-Add Resource

\begin{tabular}{lcccc}
\hline $\begin{array}{l}\text { Modeled } \\
\text { Location }\end{array}$ & $\begin{array}{c}\text { Regional DR } \\
\text { Potential (MW) }\end{array}$ & $\begin{array}{c}\text { DR Time } \\
\text { (h) }\end{array}$ & $\begin{array}{c}\text { Regional Load Add } \\
\text { Potential (MW) }\end{array}$ & $\begin{array}{c}\text { Load-Add } \\
\text { Time (h) }\end{array}$ \\
\hline Seattle, WA & 7.89 & 2.5 & 2.39 & 3 \\
\hline Spokane, WA & 1.66 & 2.5 & 0.45 & 3 \\
Portland, OR & 6.69 & 3 & 2.45 & 3 \\
Boise, ID & 5.19 & 2.25 & 1.40 & 3 \\
Total & $\mathbf{2 1 . 4 3}$ & - & $\mathbf{6 . 6 9}$ & - \\
\hline
\end{tabular}




\section{Discussion}

Knowledge derived from field testing, in combination with additional information gained from the use of calibrated models, provided a detailed understanding of the issues and potential associated with low-temperature supermarket DR. The following sections explore the lessons learned from this work and their implications for future work in this area.

\subsection{Thermal Dynamics of Refrigerated Display Cases and Walk-Ins}

Tests at TAN and the modeling performed provide a good understanding of thermal characteristics of refrigerated cases and walk-ins. First, initial tests (see Section 4.1) established a rate of temperature increase in reach-in cases and walk-ins when refrigeration was suspended. The reach-in cases were found to warm at a rate of approximately $10^{\circ} \mathrm{F} / \mathrm{h}$ when cooling to the case was suspended, and the walk-ins at a rate of approximately $2^{\circ} \mathrm{F} / \mathrm{h}$. This rate was found to be fairly typical for other walk-ins as well, but is a function of case/walk-in geometry, size, etc.

These rates have a few implications for any DR strategy that is to be used in real world application. First, in order to achieve the maximum load shed, compressors need to be shut off completely. Assuming low-temperature cases and walk-ins are served by the same rack, that normal operation is at a case temperature of $0^{\circ} \mathrm{F}$, and that the maximum case temperature allowable for food safety and owner approval is $15^{\circ} \mathrm{F}$, these initial experiments showed that the maximum power savings are available for only an hour and a half (without precooling).

The second aspect of the thermal dynamics of supermarket equipment that was investigated was the relative response of cases and walk-in air and product temperatures after set point changes were applied. As can be seen in Section 5.2.1, case temperature, which is normally the control variable that dictates compressor operation, changes roughly six times faster than the product simulator temperature did in our experiments, owing to its much smaller thermal capacitance. This suggests a different control variable may be more appropriate for DR events, and would allow them to continue for much longer than if bounds were placed on case air temperature as is done during normal operation.

Another consideration that arises in light of this fact is that suction pressure control would be much different if governed by the product temperature rather than the case air temperature. The current practice is to allow suction pressure to float in response to the case air temperature. If the air temperature is only a surrogate for the product temperature, which is the temperature that is really being controlled, newly available product simulators may allow additional load shed by allowing higher suction pressures for longer during DR events. All simulations performed as part of this project assumed floating suction pressure was controlled by the air temperature of the critical case. If this control was moved to product temperature, more and longer savings are likely.

\subsection{Available Load Shedding for Demand Response}

Modeling and field tests were used to quantify the amount of load shedding possible in BPA's service territory. Field evaluation tests alone were insufficient for such a quantification for a few reasons. First, less than half the capacity of a single rack could be modified because of product safety concerns. This was mostly due to the fact that ice-cream cases, walk-ins that held ice cream, and medium-temperature cases and walk-ins were all served by the same rack as the low- 
temperature cases and walk-ins. Secondly, submetering was only available at the rack level, meaning there was a great amount of noise present in the data. Furthermore, the baseline, which was used to quantify DR savings, was generated using a statistical model of the previous 6 weeks' operating data, rather than power actually measured under identical conditions. This, combined with the fact that only about a quarter of the capacity of the refrigeration system was available for DR testing and that testing was not necessarily performed during the hottest days of the year, led to a situation in which the large fluctuations in baseline power were sometimes greater than the estimated savings, obscuring results.

Lastly, tests were performed when possible - on days when equipment was operating properly and technicians were available to make changes, rather than the days on which an actual DR event was likely to occur in practice. As can be seen in Section 5, the total power draw of the rack and thus the available load shed is often much greater the hotter the outdoor conditions. Thus, tests performed on cooler days may not reflect the maximum load shed possible under more extreme conditions.

These issues were in large part addressed through the modeling study included herein. Further testing in a laboratory where conditions could be controlled and metering performed at a finer resolution would add significant value to this study. Despite the issues discussed above, the team was able to demonstrate a load shed of around $10 \mathrm{~kW}$ for more than 2 hours in the most effective DR tests. Calibrated modeling showed that demand savings of $15.0-19.8 \mathrm{~kW}$ are available for 1.5 hours for a typical store without precooling and for around 2.5 hours with precooling throughout most of BPA's service territory.

The availability of this amount of load shedding requires a few strategies to be in place. First, the team found fairly conclusively that simply raising the saturated suction pressure of racks on which this was permissible was insufficient to shed substantial power. Cooling load must be shed as well by raising the control set points of individual circuits. Precooling product may increase the amount of power shed available or extend the time it is available, but no definitive conclusions can be drawn from the field test on this variable.

This amount of load shed available is also lower than earlier estimates. There are a couple of reasons for this. First, the relationship between product temperature and power draw for refrigerated display cases was not known before this project. In most cases, the product temperature reached its maximum value and either the DR test had to be stopped or a modified control strategy that kept the product near its maximum for a certain time period was employed. Second, a large portion of the refrigeration system in the store was not available for testing during this project due to owner concerns about product quality. If medium-temperature cases were included, additional DR savings would be realized.

\subsection{Available Load Add}

Another analysis was performed to quantify the amount of load that could be added to the grid by an individual supermarket when energy is plentiful. The simulations were run at 3 a.m. on April 21, 2014. They showed that 5.2-5.5 kW of demand could be added for 3 hours per store, depending on location. This calculation is heavily dependent on some of the assumptions made in the study. The most important of these is that normal operation of the cases is at $80 \%$ of 
maximum capacity. This puts a precise number on the amount of reserve capacity available, which may be greater or lesser based on the equipment that an individual store selects.

\subsection{Practical Challenges}

Pilot testing of DR strategies is a key component of a three-pronged approach to developing successful strategies with broad impacts (the other two components are numerical simulation and laboratory testing). In-store evaluation tests identify key stakeholder concerns and integration challenges and also test initial idealized assumptions. Pilot testing in Portland, Oregon, has shown that to achieve a significant DR using supermarket refrigeration systems requires overcoming several technical, logistical, and nonenergy challenges. These challenges are enumerated and discussed below.

\subsubsection{Medium-Temperature Cases}

Medium-temperature refrigeration systems as currently configured are not available to provide a DR (or demand-add) resource. These systems typically serve protein-rich products such as dairy and deli and are kept just above freezing. They cannot be precooled to store extra cooling energy prior to a DR event or to increase the electricity load during a load-balancing event because that would risk freezing the product. These products also cannot be warmed above their typical operating set points. They are already above freezing, so any time spent at warmer temperatures poses potential product quality and health issues.

It is hard to overstate the degree to which this constraint affects this study and any subsequent DR program. Figure 26 shows the refrigeration electrical load at $90^{\circ} \mathrm{F}$ condensing temperature, by case type, for the U.S. Department of Energy Reference Supermarket. The red portion is the portion that the team assumed was available for the modeling studies in this report—namely, the low-temperature, non-ice-cream cases. As is easily seen, this is a small portion of the total capacity of the store. Additional strategies must be devised to take advantage of the mediumtemperature cases (shown in green) and possibly the ice-cream cases (shown in blue). 


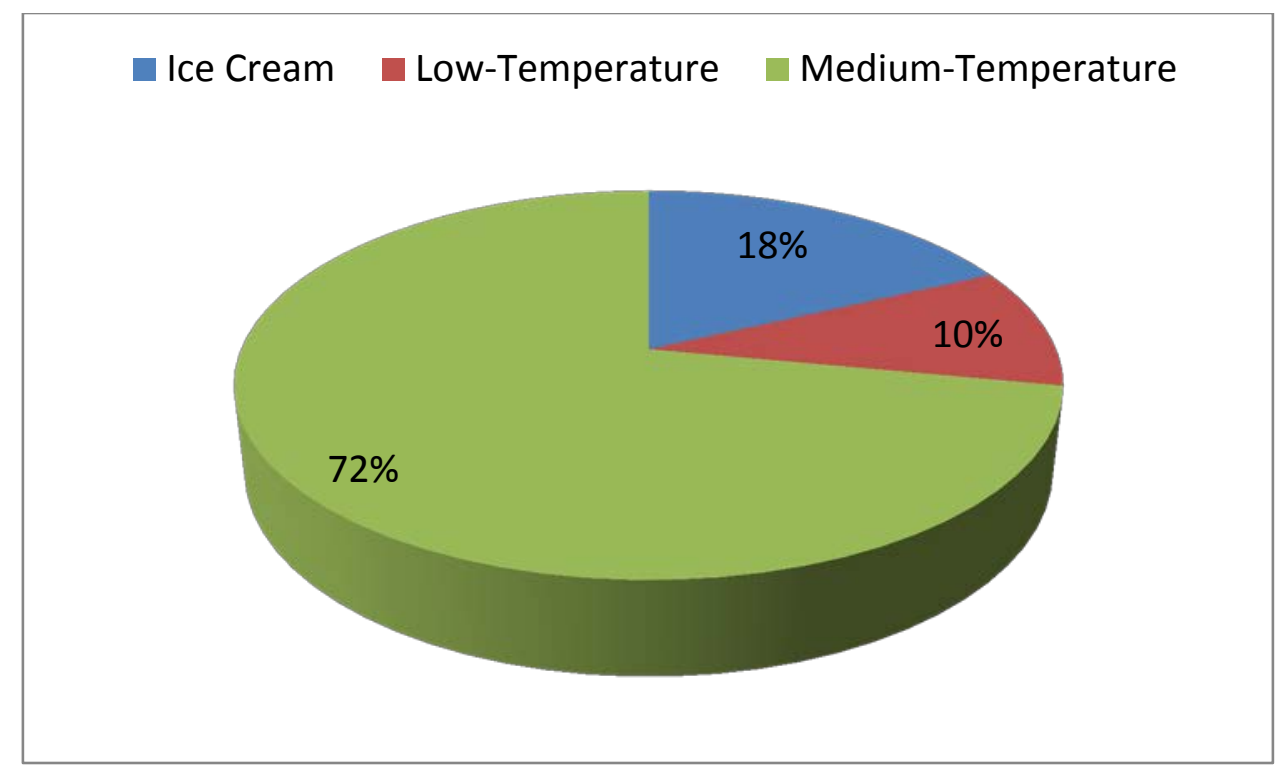

Figure 26. Reference supermarket refrigeration electricity load at $90^{\circ} \mathrm{F}$ condensing temperature, by case type

One strategy that may allow medium-temperature cases to be employed for DR purposes is the inclusion of a secondary loop that can be charged during off-peak hours, possibly by making ice or precooling a working fluid. This secondary loop could be discharged during the DR event without any effect on the product temperature, while shedding the electricity load of the cases.

Another strategy that may allow the use of medium-temperature cases, but would require additional control logic, would be to cycle the medium-temperature compressors in a manner similar to what's done for large-scale residential air conditioner DR. Using this strategy, no single compressor would be off for more than, say, 5 minutes, but at any given time, only a fraction of the medium-temperature compressors would be operating.

A related concern is that the supermarket owner the team worked with was not interested in testing demand-add strategies (increasing power consumption for the purposes of balancing variable production without other ancillary benefits) because it was perceived to be at odds with their mission to save energy and risked increasing their demand charges.

\subsubsection{Low-Temperature Cases}

The typical configuration of low-temperature refrigeration systems also works against the objective of achieving a large DR. Low-temperature systems serve reach-in refrigerated display cases and walk-in freezers that contain a range of products that require different levels of refrigeration. Ice cream, due to its high sugar and fat content, needs to be kept much colder than other frozen products - below $-5^{\circ} \mathrm{F}$ - to prevent melting. Therefore, a common control strategy is to run the entire low temperature refrigeration system to maintain an air temperature of between $-8^{\circ} \mathrm{F}$ and $-10^{\circ} \mathrm{F}$ and throttle the refrigerant flow (using evaporator pressure regulators) to noncritical items to achieve an evaporator discharge temperature closer to $0^{\circ} \mathrm{F}$.

More recently, refrigeration design has evolved to include finer subdivision of suction groupings to save energy. However, most existing low-temperature systems are subject to the refrigeration 
needs of the coldest items, limiting DR flexibility. One commonsense strategy is to raise the suction pressure of the low-temperature system, lowering the temperature "lift" that the system must achieve, shedding load, and reducing power consumption. However, although most products can tolerate a temperature rise to $15^{\circ} \mathrm{F}$, the rack temperature can only be raised to the point at which ice cream risks melting $\left(-5^{\circ} \mathrm{F}\right)$. This reality limits both the amount of power that can be shed and the duration of the event. When low-temperature systems are grouped by the set points required by different products and by reach-in cases versus walk-in freezers (which stay cold much longer than reach-in cases), supermarket owners will have much more flexibility in controlling their systems to achieve DR.

\subsubsection{Controllability}

Refrigeration systems are currently controlled for steady-state operation. For example, the evaporator pressure regulator valves used to throttle refrigerant flow are typically set with a screwdriver at a fixed value. This prevents precooling of noncritical cases and also prevents raising the set points of these noncritical refrigeration circuits to shed load on the system. However, EEPRs are available that can be used to control the evaporator DAT to provide more flexibility to the system operator to add or reduce load on the system.

A related issue is the ancillary equipment in refrigerated reach-in and walk-in cases such as lights, anti-condensate heaters, and fans. In the stores the team worked with, this equipment could only be controlled on or off, and therefore was not available to serve as a DR resource. These loads represent a significant fraction of the total power consumption of the refrigeration system and also generally add heat to the refrigerated space, working against DR strategies that rely on a very gradual rate of warming when compressor power is reduced. It would be beneficial to use the refrigeration system and the ancillary case equipment in concert when managing loads for DR.

\subsubsection{Compressor Noise}

Despite the focus on steady-state operation, the control of low-temperature refrigeration racks is typically very noisy, in a statistical sense. Capacity control is delivered in most existing lowtemperature refrigeration systems by staging multiple large single-speed compressors, meaning that step changes up or down of 10 to $15+\mathrm{kW}$ are common. This noise in the power signal is problematic for two reasons. When calculating DR, a statistical baseline must be calculated representing what power consumption would have been in the absence of the DR. The uncertainty of this baseline - and therefore of the achieved DR - is directly related to the noise in the power signal. A large DR will be obscured by this background noise, even when the lowtemperature refrigeration compressor rack is separately submetered.

The problem becomes even greater if the main service electricity meter is being used to calculate the DR signal, because the noise of the building's HVAC system, medium-temperature refrigeration system, and equipment must also be accounted for. Due to the additional cost, supermarkets may be unwilling to submeter their stores at a higher level of detail without financial incentives. Also, the power shed during a DR event can be undermined when a large compressor is staged on to meet a very small refrigeration requirement. Variable capacity control-whether using a digital discus technology on a reciprocating compressor, variable frequency drive, or compressor technology designed for variable capacity like a digital scrollshould smooth out the power baseline and better match power consumption to load, as well as 
deliver a more stable refrigerant temperature. This last feature is of great value to supermarket owners because it results in extended product longevity. Two of the stores available for pilot testing in Portland were retrofit in the summer of 2015 with variable capacity compressors and EEPRs, but they were not back online in time for DR testing for this project.

\subsubsection{Practical Considerations}

There are also practical challenges to pilot testing in real stores. Maintaining smooth store operation, customer satisfaction, product integrity, and above all food safety are the primary concerns of the owner and store management. Therefore, new control strategies such as using product temperature simulators as control points rather than evaporator DAT require careful monitoring as well as the presence of a refrigeration technician to initiate the test, monitor the integrity of critical items, and terminate the test and return the system to its original operation. The hot summer conditions required for key DR testing coincide with the highest demand for refrigeration technician services (many refrigeration systems succumb to the strain induced by heat waves), so it can be challenging to successfully schedule and execute tests of more innovative DR strategies.

In-store pilot testing is most appropriate for tests that have either already been shown in a laboratory to be low risk or tests that only involve current control points and equipment, and that keep evaporator DAT within bounds deemed safe by the store owner. In this second case, it is straightforward for the test to be initiated remotely without the need for close oversight because the refrigeration set points return to their default values after a timed override. Although in some cases retrofits to the refrigeration systems benefited this project - the addition of EEPR valves and variable capacity on the lead compressor on the $-20^{\circ} \mathrm{F}$ suction group at TAN, for examplethe timing of other retrofits and failed compressors were problematic. For example, the refrigeration racks at two additional potential evaluation test sites were both replaced during the summer of 2015, meaning the stores were offline when pilot testing was scheduled to occur. A refrigerant leak in the lead compressor on the new variable capacity lead compressor at TAN (not caused by this project) meant that the power consumption of the low-temperature refrigeration system was even noisier than before the retrofit.

These logistical challenges are compounded by the remote nature of the testing. It is more challenging to coordinate a field test from abroad that involves several dispersed actors, compared to laboratory studies or local field testing, even with remote access to data from the refrigeration controller and electrical submetering systems. In summary, remote pilot field testing is most appropriate for rolling out previously developed strategies that require little intervention on the part of company staff or refrigeration technicians, and less appropriate for developing new strategies.

\subsection{Considerations for Choosing a Demand Response Strategy}

In general, DR using refrigeration systems requires a tradeoff between the amount that refrigeration power can be reduced and the length of time it can be reduced. For example, it may be tempting to adopt a strategy that completely shuts off the low-temperature refrigeration system compressors. This strategy will shed the maximum amount of power, but the critical cases will warm at the maximum rate, limiting to about 45 minutes the amount of time this strategy is effective before the system rebounds to cool the critical products. On the other hand, 
if refrigeration is maintained, but set points are raised, reducing the load on the system, it is possible to provide less DR but for an extended period, on the timescale of hours.

\subsection{Additional Observations}

One additional observation is that currently, DR using refrigeration systems is focused mainly on using the thermal mass of the frozen products. In other words, strategies leveraging current refrigeration systems rely on storing "sensible" heat in the frozen products (in other words, not relying on the "latent" heat associated with phase changes). There are emerging technologies using tunable phase change materials for both medium- and low-temperature systems that could significantly expand DR capabilities if they are found to be technically and economically sound. The team recommends that special attention be paid to the heat transfer coefficients in this type of application to ensure that heat can be effectively transmitted to the phase change material.

One aspect of the DR equation that was not addressed in this study is the automation of DR and the increase in available DR sources that may be achieved through optimized control.

Supermarket owners and refrigeration technicians, two key stakeholders, are very comfortable with temporary, timed set point overrides that revert to default values after a period of time. However, allowing a controller in the store or in the cloud to make optimal decisions in real time may significantly affect the size of the available resource.

This could occur through preprogrammed logic in a controller that selected an appropriate strategy based on price signals, outdoor conditions, and user preferences. A more promising strategy, however, may be integrating models of particular supermarkets into the decisionmaking tools. These models can "learn" from the operation of the supermarket by gathering data on relationships between operating parameters and electricity use. Then they would be able to make optimal decisions about whether and how to execute DR based on an analysis of many different scenarios done in real time just before the chosen strategy was executed. 


\section{Conclusions and Future Needs}

Through a set of field tests and a detailed modeling study, the team determined that a reasonable expectation for the amount of supermarket load shedding that may be offered by manipulating only low-temperature cases (and excluding ice-cream cases) is on the order of 15 to $20 \mathrm{~kW}$ in BPA's service territory. Extrapolated to the entire region, this represents an aggregate potential on the order of 21.4 MW within BPA's service territory. This DR resource should be available for an hour-and-a-half with no precooling, and for more than two hours when cases are cooled below their normal temperatures before the DR event.

Inability to shed refrigeration load for medium-temperature products is the largest barrier to realizing more significant supermarket DR. The next largest barrier is lack of control over ancillary reach-in case equipment, although separating refrigeration suction groups further to better match the needs of different products would help address this issue. Additionally, greater use of variable-capacity compressors would provide a smoother baseline for benchmarking DR delivered and would allow the refrigeration system to better match small loads during DR, avoiding large spikes during a shed event.

Based on this study, the team proposes the following modifications be done at supermarkets wishing to participate in DR programs:

1. Install EEPRs on all circuits to be used in DR programs.

2. Assemble cases into suction groups that allow greater freedom to vary set points. Currently, it is common for the entire low-temperature system to be slaved to critical case control, rather than zoned such that cases/walk-ins with similar refrigeration loads are grouped together.

Moving the lowest-temperature circuits (ice cream) to a dedicated refrigerant loop would provide permanent energy efficiency in the form of a higher suction temperature for other low-temperature cases, and allow DR strategies to include a much larger increase in SST on non-ice-cream cases.

3. Enable floating suction control and possibly even steady-state case-level control to be switched between case air temperature and product simulators. The case air responds much more rapidly to changes in operation, which can be detrimental to DR strategy.

4. Implement variable capacity compressors to maintain low and predictable power consumption during DR events.

5. Enable refrigeration lights, fans, and anti-condensate heaters to ramp down for DR in concert with the refrigeration system. This would significantly increase the DR resource available.

6. Better insulate reach-in cases to decrease external thermal loads.

The team also identified several research needs that would increase the amount of DR available in supermarkets:

1. Conduct a study of DR using medium-temperature cases. Although the team believes that there is a great deal of DR potential that can be realized from medium-temperature refrigeration systems, it seems that identification of the size of this resource and the 
control strategies necessary will require laboratory testing. Performing tests in an actual supermarket requires convincing store owners to allow manipulation of cases with valuable meat and dairy products, which are sensitive to temperature changes. Laboratory tests would help address this hurdle.

Laboratory tests should likely focus on ways to cycle medium-temperature compressor operation so that (1) only a fraction of the compressors in a given store or region are on at a given time, and (2) the effect on case temperature is minimal. This is similar to strategies used for residential air-conditioning DR.

2. Conduct both real-world and modeling studies to determine the coupled effect of the variation of HVAC set points and refrigeration set points. A viable DR strategy will likely include reduction in refrigeration compressor power, anti-condensate heaters, lighting, fans, and HVAC. Currently, the coupling of these changes is not fully understood.

3. Conduct research in concert with food safety experts to better understand the constraints under which DR should be conducted in regards to food safety. The constraints used in this study were dictated by one particular supermarket owner and were likely conservative. Very little research has been conducted to understand how short-term changes in temperature affects food quality. This type of research may lead to greater DR savings.

4. Further investigate the relationship between adding load at times when power is readily available and reducing power when it is not. Very little work was done on "power-add" strategies in this study in deference to the store owner's wishes. Integration of renewable sources of power with the grid will require more studies of this sort.

5. Research active phase change solutions to augment dispatchable thermal storage capabilities. This includes identifying strategies for heat transfer enhancement in phase change materials and identifying strategies for integrating these materials into refrigerated display cases and walk-ins.

This study provided an estimate of the amount of power that may be shed by manipulating typical low-temperature refrigeration systems in supermarkets in the northwestern United States. Power shedding potential was evaluated through nine real store DR field tests. Additional field tests were performed to inform the development of and calibrate a set of gray-box refrigeration models that allow the field-test results to be readily applied to supermarkets across the Pacific Northwest. These field-test results and models will be invaluable for future research in this area. Optimal strategies for including supermarkets in a larger portfolio of grid resources will likely need to include not only refrigeration systems, but also HVAC, lighting, and energy storage systems, as well as detailed algorithms for increasing and decreasing overall electric demand. Finally, the team believes significant opportunities exist for further supermarket refrigeration DR savings, although the necessary modifications require additional modeling and testing. 


\section{References}

Bienert, Rolf, and Barry Haaser. 2015. "Understanding OpenADR 2.0." Accessed April 10, 2015. http://www.openadr.org/assets/docs/understanding $\% 20$ openadr\%202\%200\%20webinar_1 1_10_11_sm.pdf.

The Cadmus Group, Inc. 2009. "Northwest Commercial Building Stock Assessment." Accessed Sept. 3, 2015. http://neea.org/docs/reports/2009northwestcommercialbuildingstock assessment021ca220f212.pdf.

California Energy Commission. 2005. "Enhanced Automation Case Study 7: Lighting and Equipment Controls/Grocery Store." CEC-400-2005-059-FS. Accessed Sept. 4, 2015. www.energy.ca.gov/2005publications/CEC-400-2005-059/CEC-400-2005-059-FS.PDF.

DOE. 2015. EnergyPlus Energy Simulation Software. Last Updated Sept. 1, 2015. Washington, D.C.: U.S. Department of Energy (DOE). http://apps1.eere.energy.gov/buildings/energyplus/.

Doebber, Ian, Adam Hirsch, E. Nelson, C. Reis, J. Armer, and J. Stein. 2015. A Circa-2013 Supermarket Refrigeration Baseline Methodology for Benchmarking Energy Performance. TP5500-63915. Forthcoming. National Renewable Energy Laboratory (NREL), Golden, CO (US).

Godfrey, K. 1980. “Correlation Methods.” Automatica: 527-534.

Hasse, H., M. Becker, K. Grossman, and Gerd Maurer. 1996. "Top-Down Model for Dynamic Simulation of Cold-Storage Plants.” International Journal of Refrigeration 19-1: 10-18.

Heaney, M., Ian Doebber, I. and Adam Hirsch. 2015. Customer Baseline Load Modeling and Uncertainty Analysis for Quantifying Demand Response in Commercial Buildings. SR-550065084. Forthcoming. National Renewable Energy Laboratory (NREL), Golden, CO (US).

Northwest Power and Conservation Council. 2005. "Fifth Northwest Electric Power and Conservation Plan." Accessed Sept. 4, 2015. https://www.nwcouncil.org/energy/powerplan/ 5/plan.

Northwest Power and Conservation Council. 2010. "Sixth Northwest Conservation and Electric Power Plan." Accessed Sept. 3, 2015. https://www.nwcouncil.org/energy/ powerplan/6/plan/.

O’Connell, Niamh, Henrik Madsen, Philip Delff, Pierre Pinson, and Mark O’Malley. 2014. Model Identification for Control of Display Units in Supermarket Refrigeration Systems.

Portland General Electric. 2013. "Energy Partner ${ }^{\mathrm{SM}}$ Customer Spotlight." Accessed August 11, 2015. https://www.portlandgeneral.com/business/medium_large/products_services/docs/albertso ns.pdf.

Shelby Publishing Co., Inc. 2015. Shelby Market Shares: West Market 2015 Data. 


\section{Appendix}

This appendix includes time-series data for simulated electricity during DR and load-add simulations.

\section{0-Minute Notice}

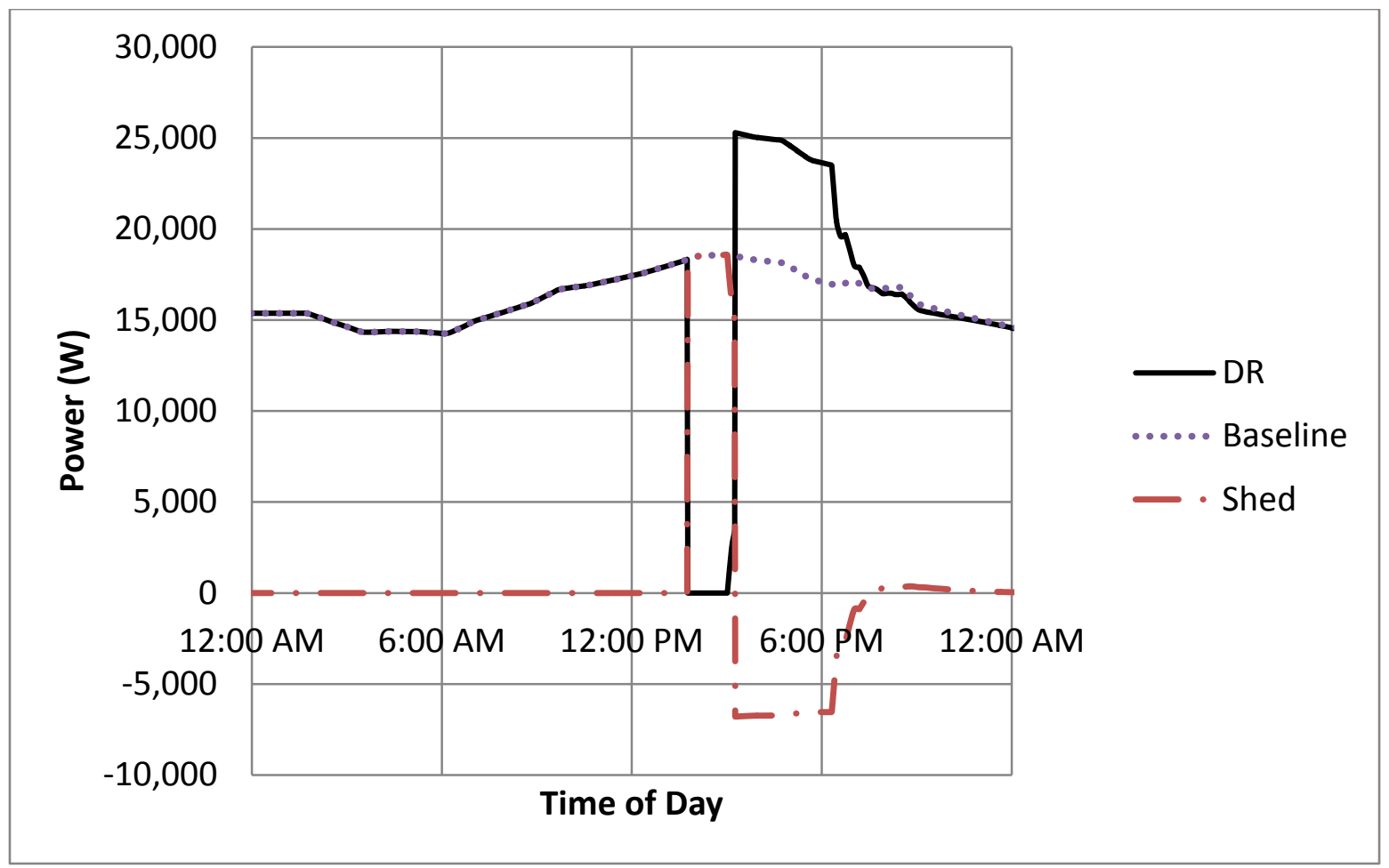

Figure 27. Simulated 10-minute notice Seattle, Washington, DR event 


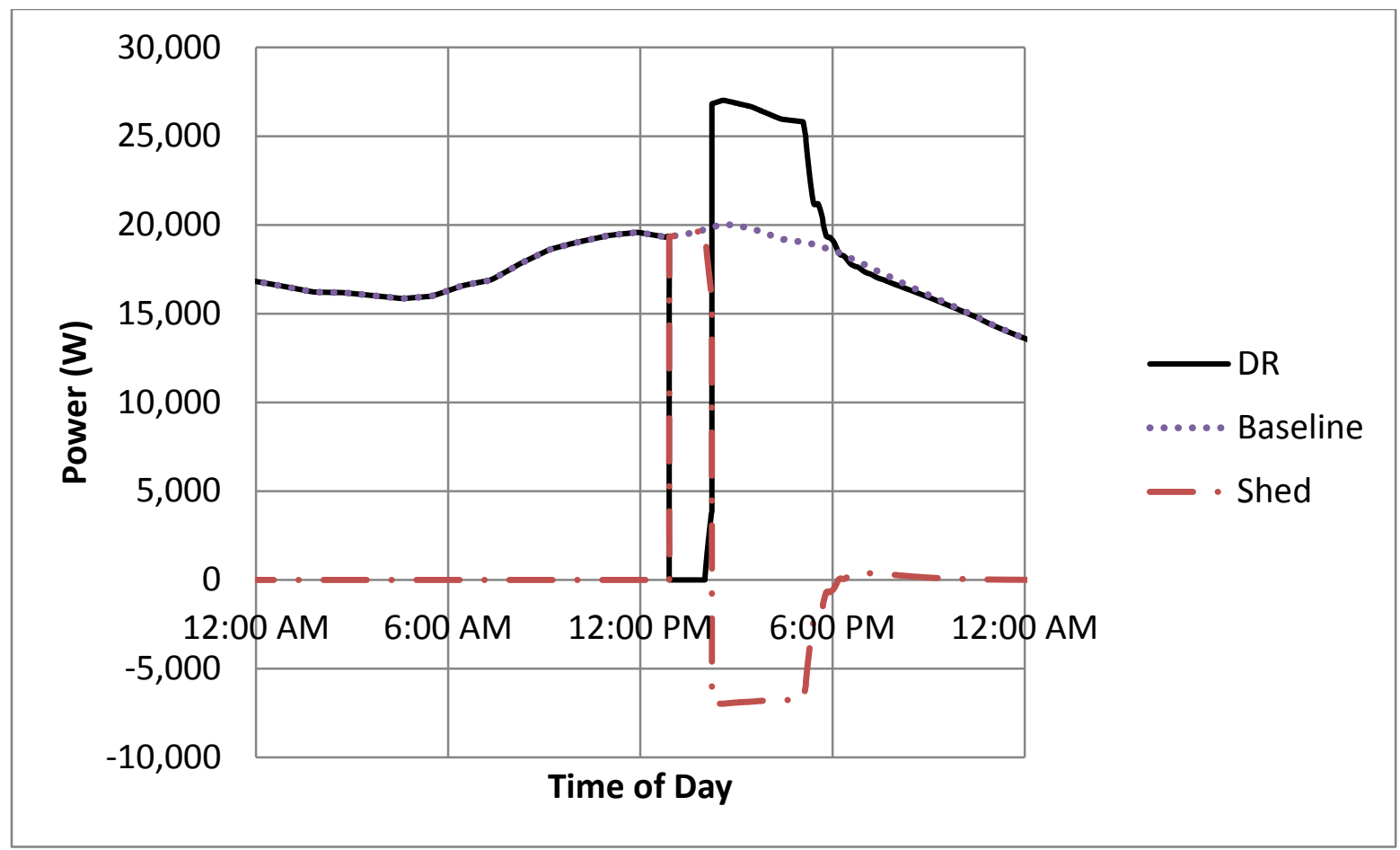

Figure 28. Simulated 10-minute notice Spokane, Washington, DR event

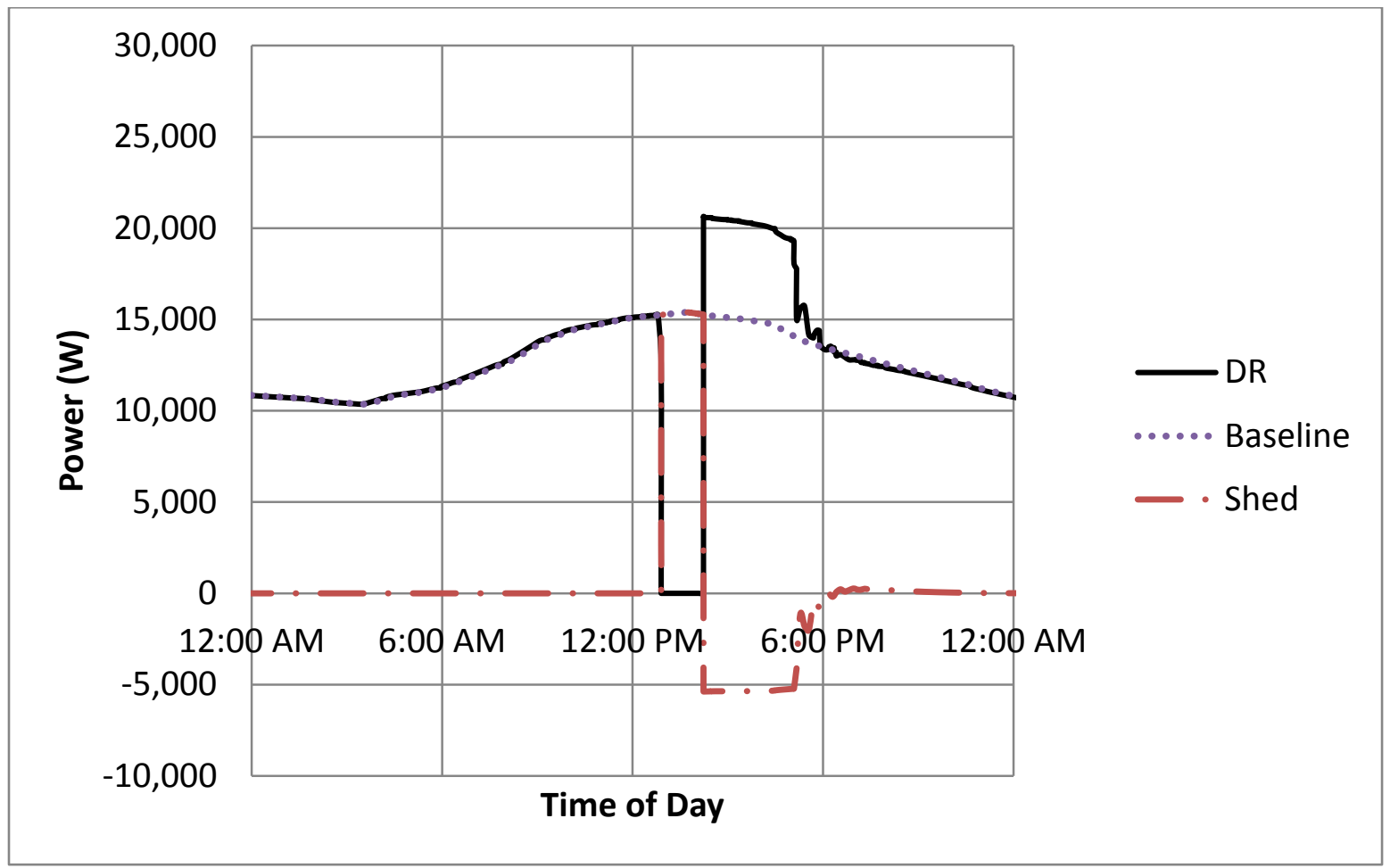

Figure 29. Simulated 10-minute notice Portland, Oregon, DR event 


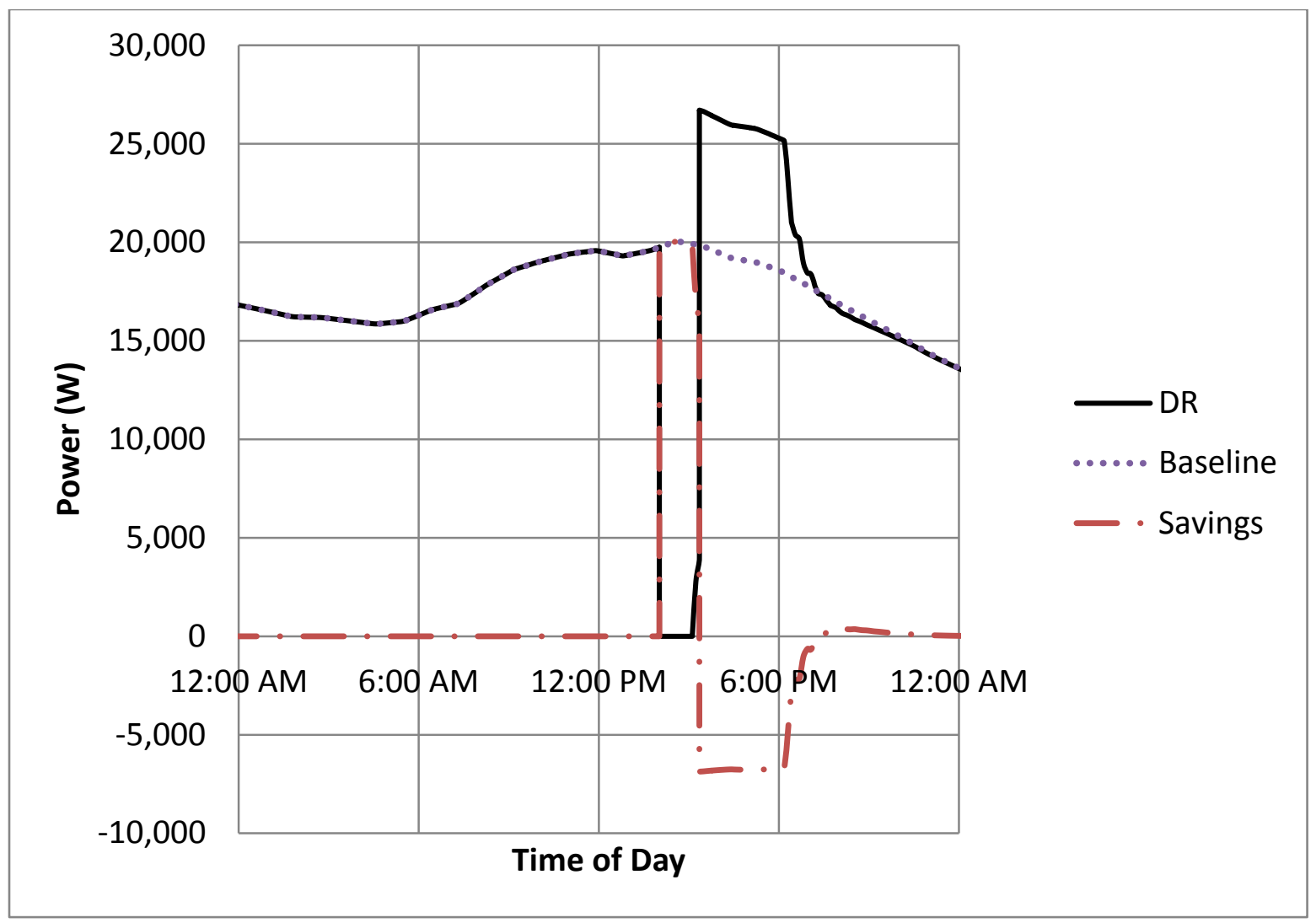

Figure 30. Simulated 10-minute notice Boise, Idaho, DR event 


\section{Day-Before Notice}

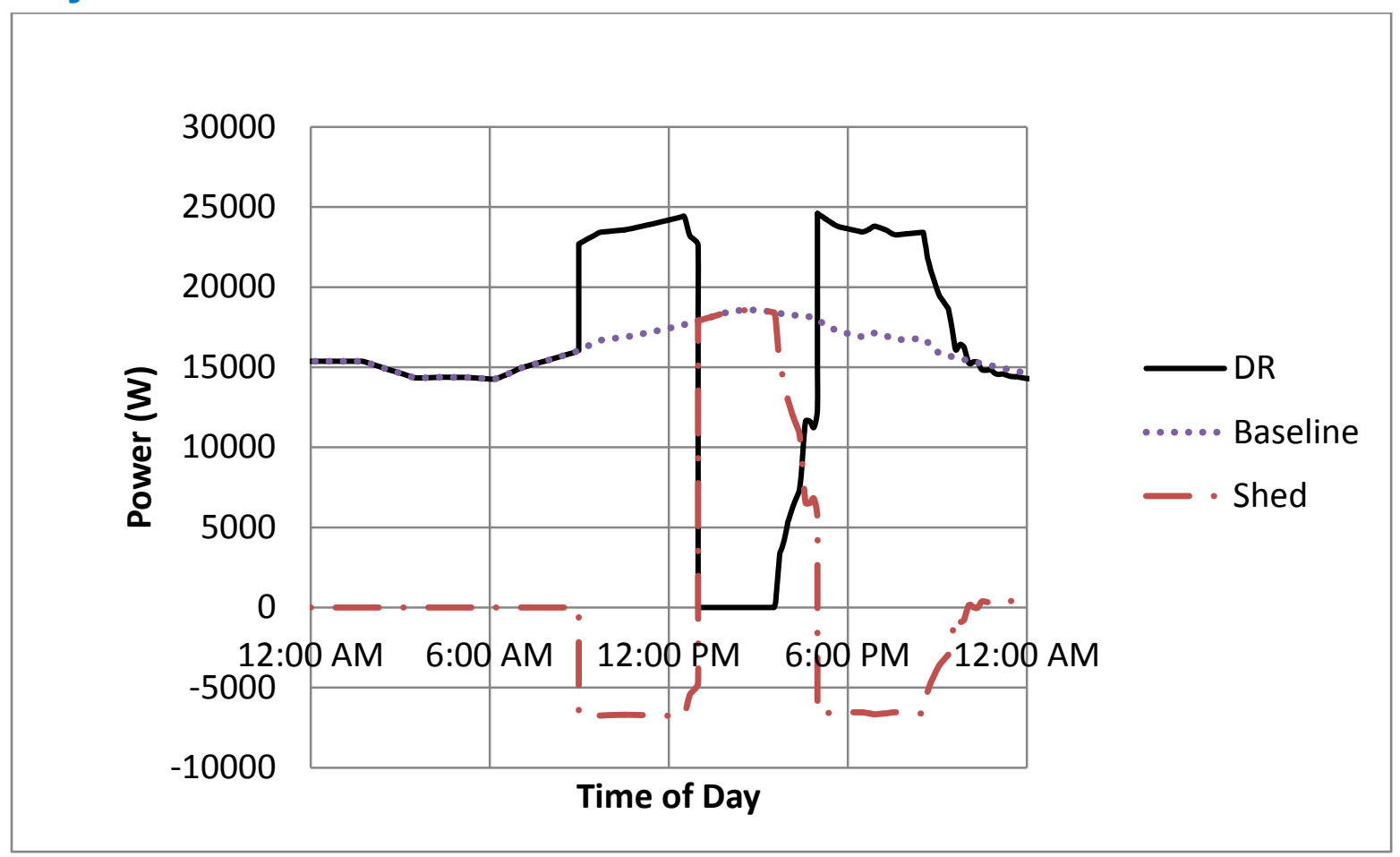

Figure 31. Simulated day-before notice Seattle, Washington, DR event

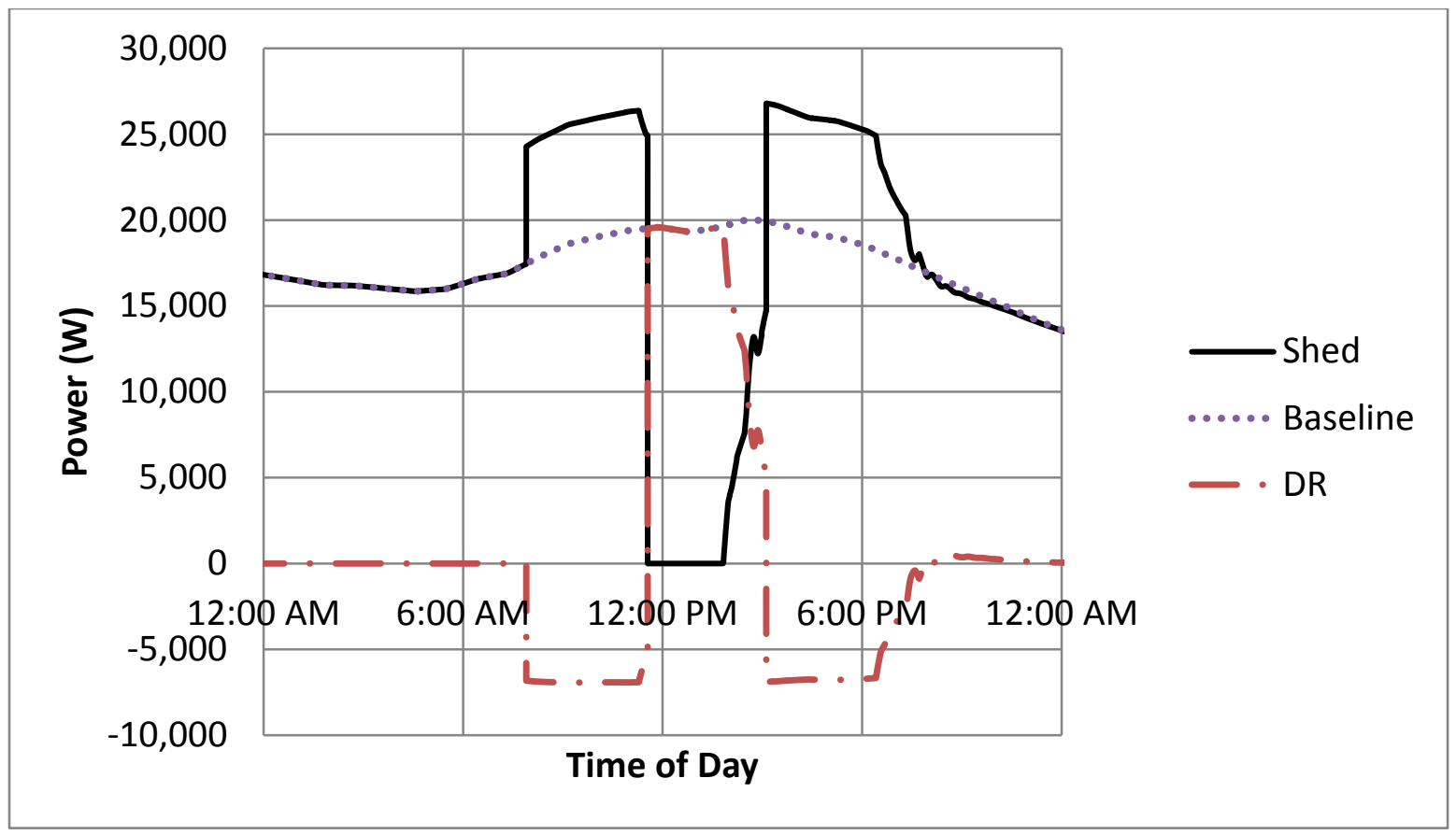

Figure 32. Simulated day-before notice Spokane, Washington, DR event 


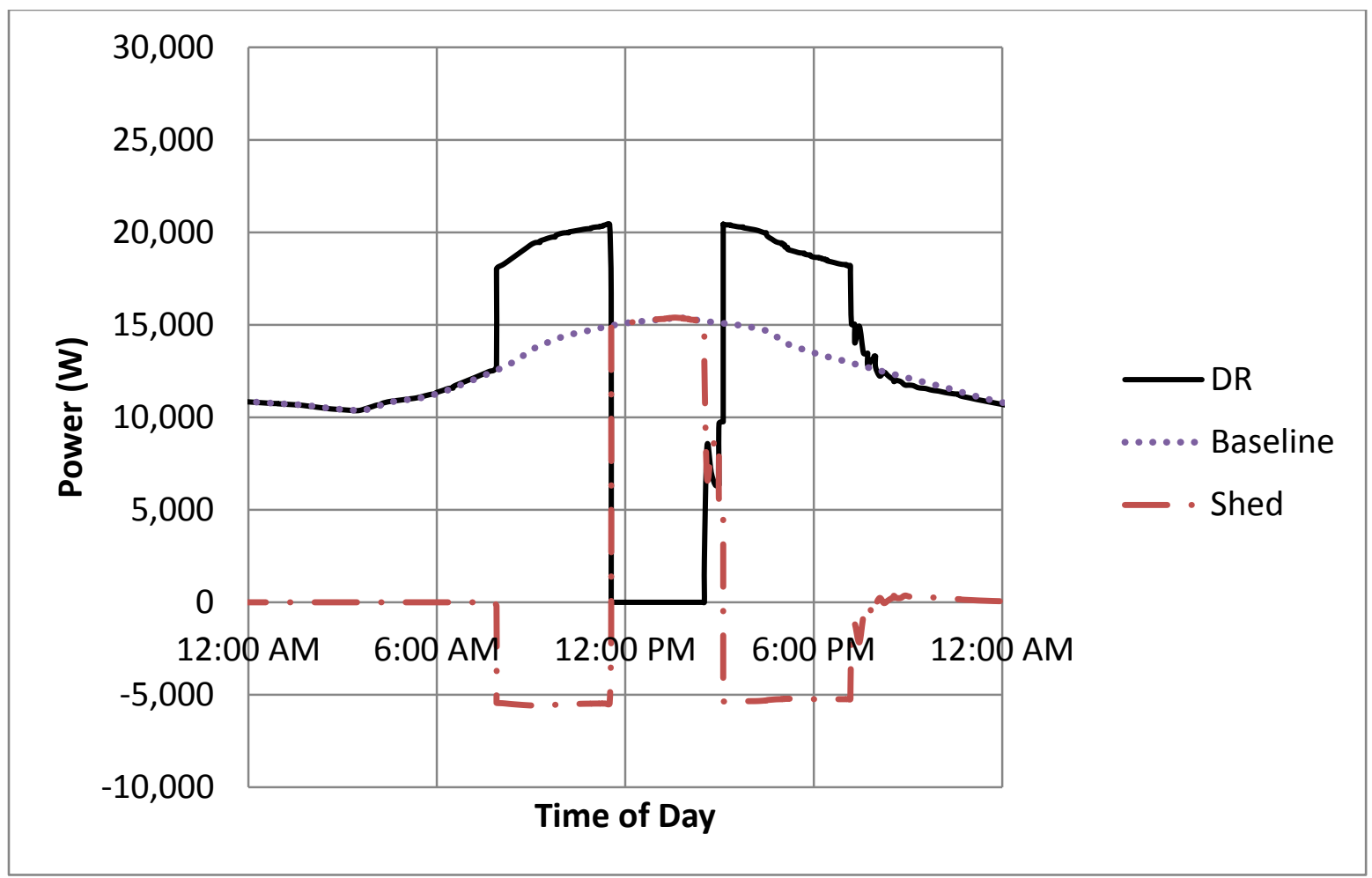

Figure 33. Simulated day-before notice Portland, Oregon, DR event

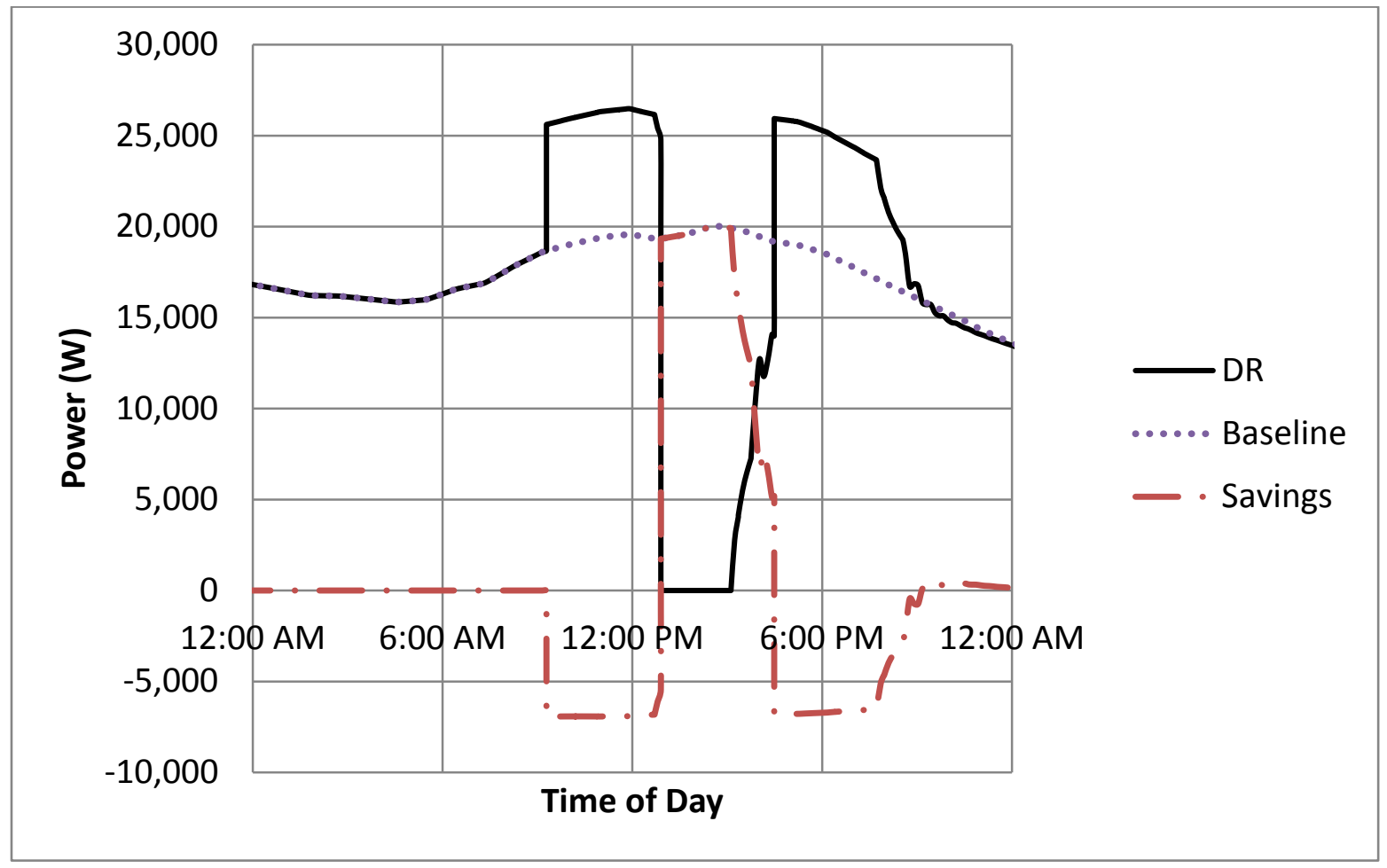

Figure 34. Simulated day-before notice Boise, Idaho, DR event 


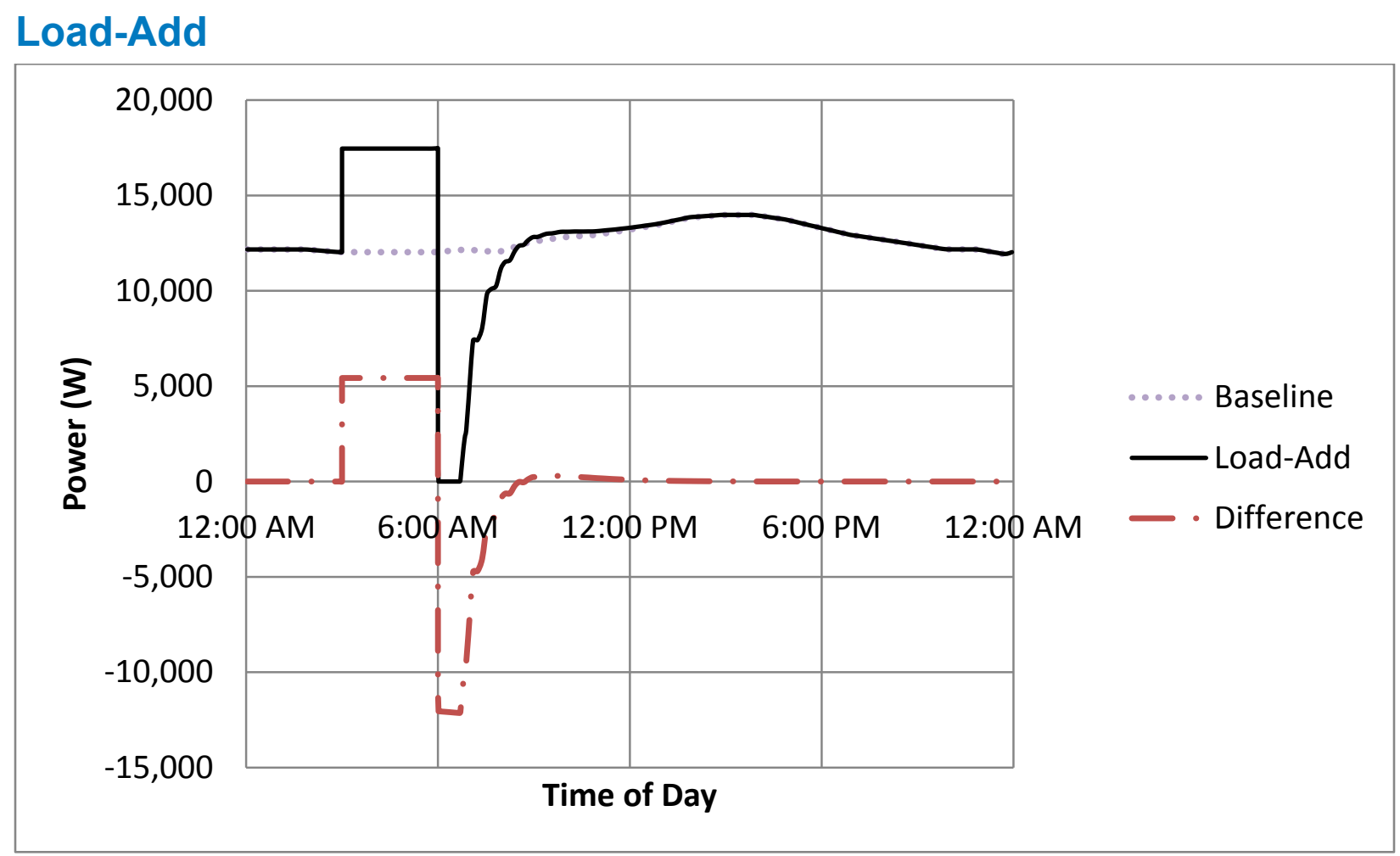

Figure 35. Simulated load-add event, Seattle, Washington

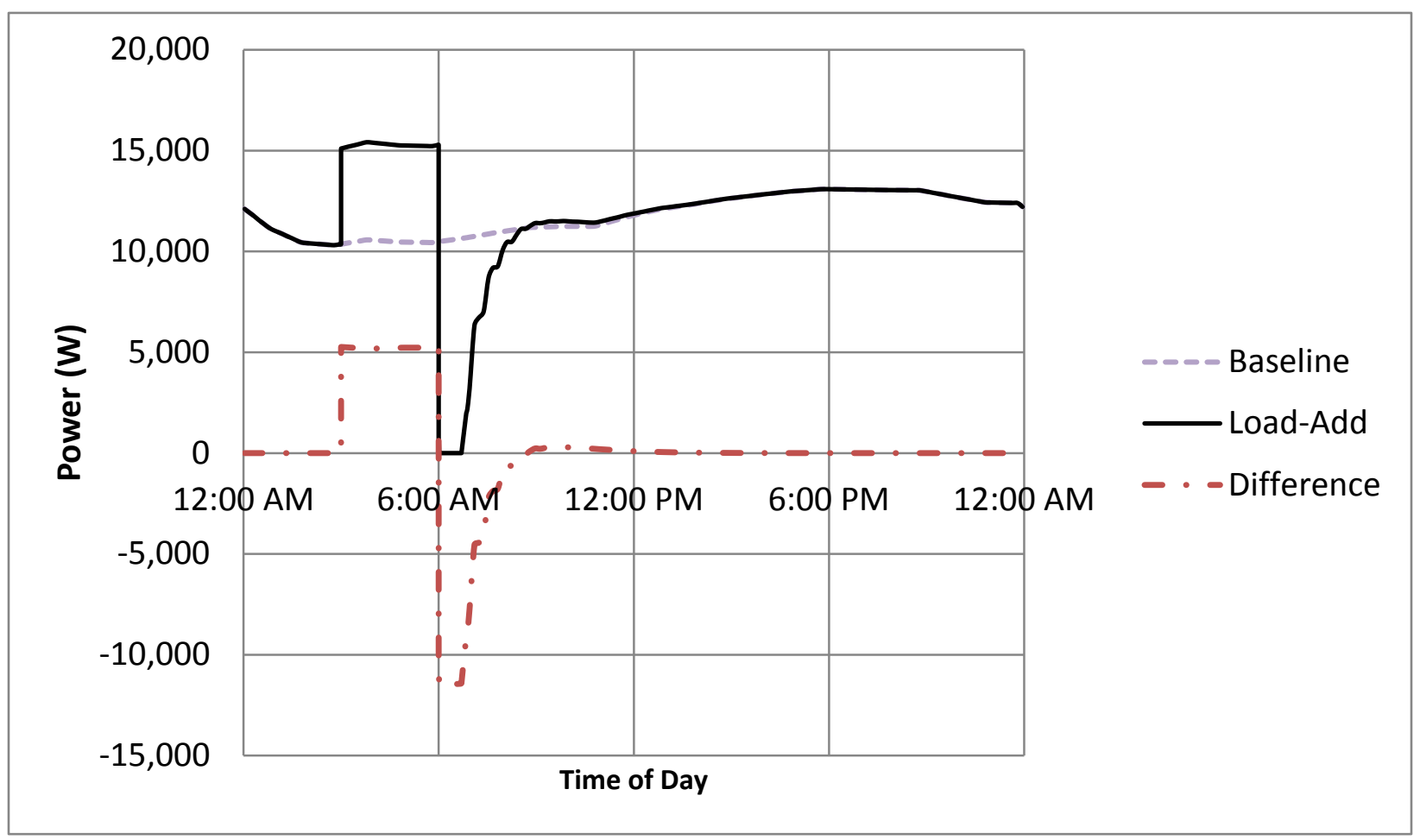

Figure 36. Simulated load-add event, Spokane, Washington 


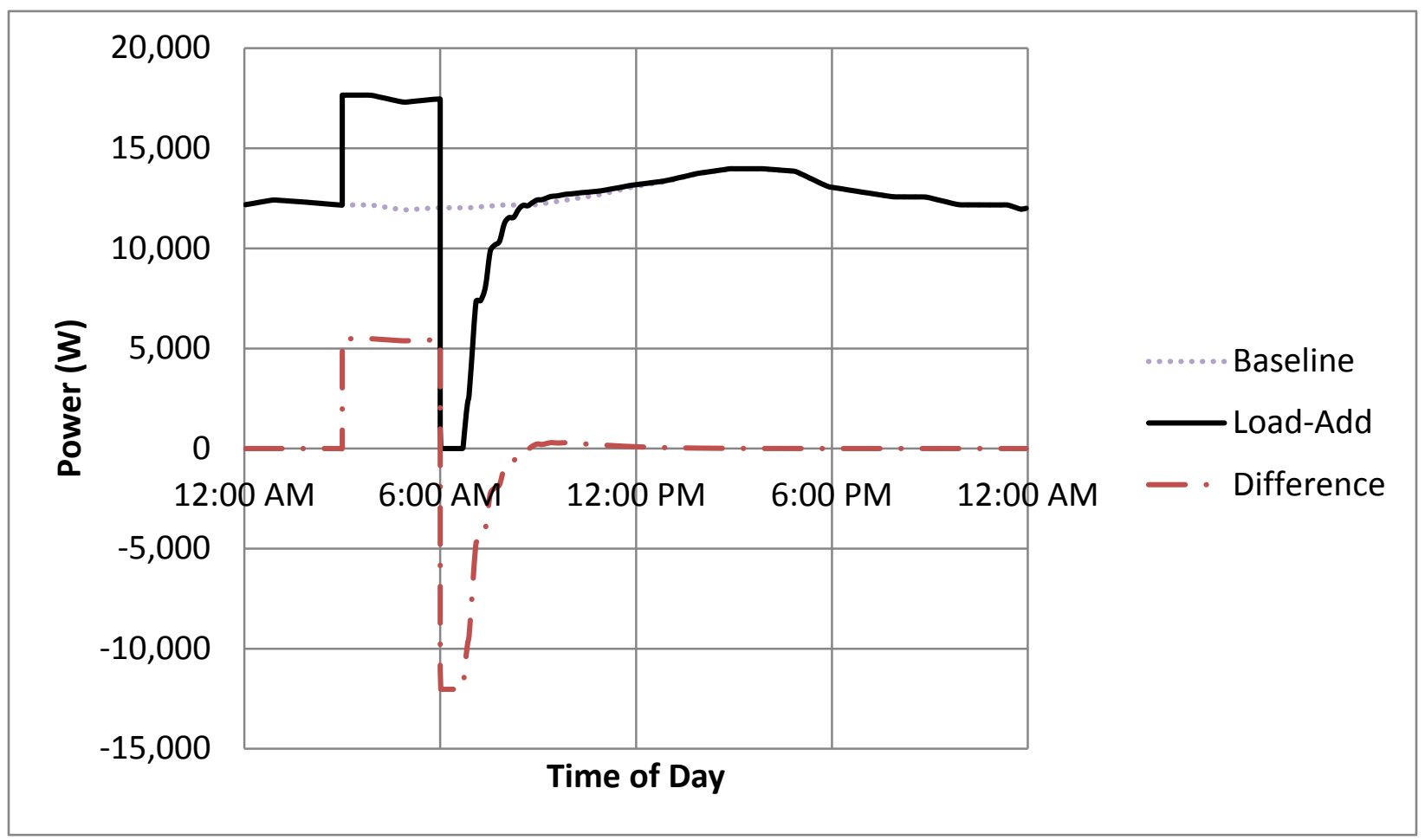

Figure 37. Simulated load-add event, Portland, Oregon

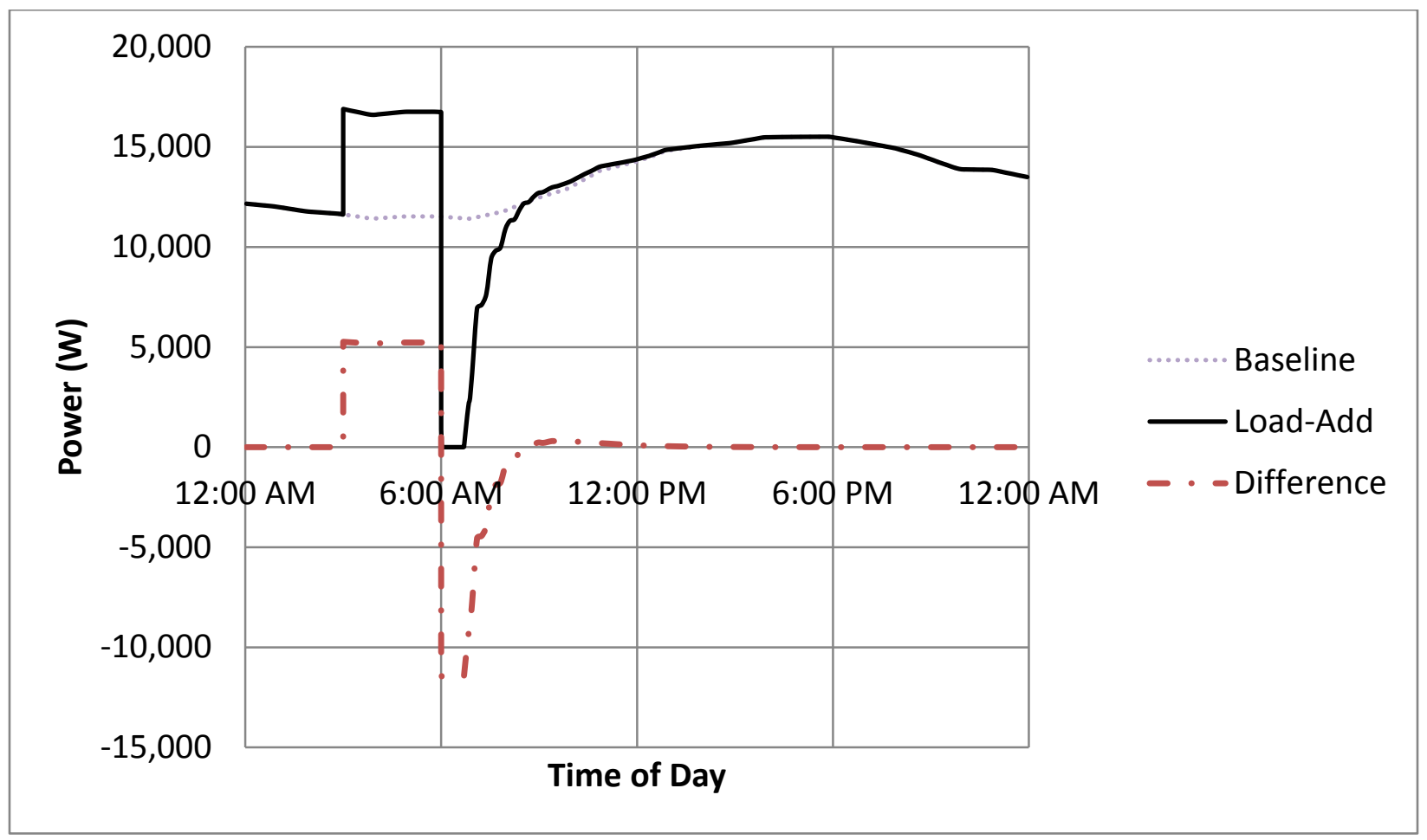

Figure 38. Simulated load-add event, Boise, Idaho 